DOI 10.4171/JEMS/278

R. Cluckers · L. Lipshitz

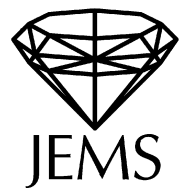

\title{
Fields with analytic structure
}

Received February 20, 2008 and in revised form August 18, 2009

\begin{abstract}
We present a unifying theory of fields with certain classes of analytic functions, called fields with analytic structure. Both real closed fields and Henselian valued fields are considered. For real closed fields with analytic structure, $o$-minimality is shown. For Henselian valued fields, both the model theory and the analytic theory are developed. We give a list of examples that comprises, to our knowledge, all principal, previously studied, analytic structures on Henselian valued fields, as well as new ones. The $b$-minimality is shown, as well as other properties useful for motivic integration on valued fields. The paper is reminiscent of papers by Denef and van den Dries [Ann. of Math. 128 (1988)] and by Cohen [Comm. Pure Appl. Math. 22 (1969)], and of the book by Fresnel and van der Put, Rigid Analytic Geometry and its Applications [Birkhäuser (2004)], and unifies work by van den Dries, Haskell, Macintyre, Macpherson, Marker, Robinson, and the authors.
\end{abstract}

Keywords. Henselian valued fields, $o$-minimality, $b$-minimality, subanalytic functions, cell decomposition, analytic structure, separated power series

\section{Introduction}

We begin with some background. Let $A$ be a Noetherian ring, $t \in A$ and assume that $A$ is $t$-adically complete. The notion of a valued field with analytic $A$-structure was introduced by van den Dries in $\mathrm{vdD}]$. The principal example is $A=\mathbb{Z}[[t]]$, and the corresponding "analytic functions" are the strictly convergent power series over $A$ (i.e. the elements of $T_{n}(\mathbb{Z}[[t]])=\left\{\sum a_{v} \xi^{\nu}: a_{v} \rightarrow 0 t\right.$-adically as $\left.\left.|v| \rightarrow \infty\right\}\right)$. The natural homomorphisms $\mathbb{Z}[[t]] \rightarrow \mathbb{Q}_{p}, t \mapsto p$, and $\mathbb{Z}[[t]] \rightarrow \mathbb{F}_{p}((t)), \mathbb{Z} \rightarrow \mathbb{Z} / p \mathbb{Z}$ give homomorphisms of $T_{n}(\mathbb{Z}[[t]])$ to $T_{n}\left(\mathbb{Q}_{p}\right)=\mathbb{Q}_{p}\langle\xi\rangle$ and $T_{n}\left(\mathbb{F}_{p}((t))\right)=\mathbb{F}_{p}((t))\langle\xi\rangle$, the rings of strictly convergent power series over $\mathbb{Q}_{p}$ and $\mathbb{F}_{p}((t))$ respectively, and thus the fields $\mathbb{Q}_{p}, \mathbb{F}_{p}((t))$ and ultraproducts of these fields are all structures in a natural way for the valued field

R. Cluckers: Laboratoire Painlevé, CNRS - UMR 8524, Université Lille 1, Cité Scientifique, 59655, Villeneuve d'Ascq Cedex, France, and Department of Mathematics, Katholieke Universiteit Leuven, Celestijnenlaan 200B, B-3001 Leuven, Belgium; e-mail: raf.cluckers@wis.kuleuven.be; URL: www.wis.kuleuven.be/algebra/Raf/

L. Lipshitz: Department of Mathematics, Purdue University, 150 North University Street, West Lafayette, IN 47907-2067, USA;

e-mail: lipshitz@math.purdue.edu; URL: www.math.purdue.edu/ lipshitz/

Mathematics Subject Classification (2010): Primary 32P05, 32B05, 32B20, 03C10, 28B10, 03C64, 14P15; Secondary 32C30, 03C98, 03C60, 28E99 
language with function symbols for the elements of $\bigcup_{m} T_{m}(\mathbb{Z}[[t]])$. This formalism was used by van den Dries to establish an analytic analogue of the algebraic quantifier elimination theorem of [Pas1] and also an analytic version of the Ax-Kochen Principle (when $A$ is a discrete valuation ring), namely, if $\mathcal{U}$ is a nonprincipal ultrafilter on the set of primes then $\left(\Pi_{p} \mathbb{Q}_{p}\right) / \mathcal{U} \equiv\left(\Pi_{p} \mathbb{F}_{p}((t))\right) / \mathcal{U}$, in this analytic language.

In [DHM] results about $p$-adic subanalytic sets were established by studying nonstandard models of the theory of $\mathbb{Q}_{p}$ in the language with symbols for the elements of $\bigcup_{n} T_{n}\left(\mathbb{Q}_{p}\right)$, the strictly convergent power series over $\mathbb{Q}_{p}$. This can be thought of as an investigation of fields with analytic $\mathbb{Z}_{p}$-structure, i.e. taking $A=\mathbb{Z}_{p}, t=p$.

In [DMM1] it was observed that if $K$ is a maximally complete, nonarchimedean real closed field with divisible value group, and if $f$ is an element of $\mathbb{R}[[\xi]]$ with radius of convergence $>1$, then $f$ extends naturally to an "analytic" function $I^{n} \rightarrow K$, where $I=\{x \in K:-1 \leq x \leq 1\}$. Hence if $\mathcal{A}$ is the ring of real power series with radius of convergence $>1$ then $K$ has analytic $\mathcal{A}$-structure, i.e. this extension preserves all the algebraic properties of the ring $\mathcal{A}$. The real quantifier elimination of [DD] works in this context implying that $K$ is elementarily equivalent to the subanalytic structure on $\mathbb{R}$ and hence $o$-minimal. See [DMM2] and [DMM3] for extensions.

Some of the results of [LR1] also fit into this context. If $K \subset K^{\prime}$ are complete (rank one) valued fields, then the separated power series $S_{m, n}(E, K)$ define analytic functions on $\left(K^{\prime \circ}\right)^{m} \times\left(K^{\prime \circ \circ}\right)^{n}$ and $\left(K_{\text {alg }}^{\prime \circ}\right)^{m} \times\left(K_{\text {alg }}^{\prime \circ \circ}\right)^{n}$, where $K_{\text {alg }}^{\prime}$ is the algebraic closure of $K^{\prime}$, and furthermore $K_{\text {alg }}^{\prime}$ has quantifier elimination in the corresponding separated $K$-analytic language. In [LR2] similar results were established for certain subrings of the $S_{m, n}$, namely the elements of $S_{m, n}(E, K)$ that are existentially definable over $T_{m+n}(K)$, and this was used to establish quantifier simplification for $K_{\text {alg }}^{\prime}$ in the corresponding strictly convergent (or affinoid) analytic language (i.e. the language with function symbols for the elements of $\left.\bigcup_{m} T_{m}(K)\right)$.

In [LR3] the notion of analytic $\mathbb{Z}[[t]]$-structure of [vdD] was extended to the separated context and this was used to establish various uniformity results on quantifier elimination, smooth stratification, Łojasiewicz inequalities and topological closure for rigid semianalytic and subanalytic sets for algebraically closed valued fields. The notion of analytic $\bigcup_{m, n} S_{m, n}(E, K)$-structure was also extended to nonstandard models.

In [CLR1, Sections 1 and 2], an, in some ways, more general framework than that of [vdD] for studying Henselian valued fields with analytic structure in analytic Denef-Pas languages was given, and used to prove a fairly general Cell Decomposition Theorem which was applied to certain questions in motivic integration. It required an analysis of terms and definable functions in one variable. This framework for Henselian valued fields with analytic structure has been further developed and applied in [Ce1]-[Ce3].

In [LR4] quantifier elimination and $o$-minimality for the field $K=\bigcup_{n} \mathbb{R}\left(\left(t^{1 / n}\right)\right)$ of real Puiseux series (or its completion $\widehat{K}$ ) in an analytic language with function symbols for $t$-adically "overconvergent" power series (for example $\sum_{n}(n+1) ! t^{n} x^{n}$ which converges on the disc $\left\{x:\|x\| \leq\left\|t^{-1 / 2}\right\|\right\}$, and hence is $t$-adically overconvergent on $K^{\circ}$ ) was established. These power series define analytic functions $I^{n} \rightarrow K$ but do not live in any $o$-minimal expansion of $\mathbb{R}($ see $[\mathrm{HP}])$. In [CLR2] these results were extended to larger 
classes of "analytic" functions on more nonstandard real closed fields. These results also required analyses of terms and definable functions in one variable.

All the above mentioned work involved the study of (usually) nonstandard fields (either Henselian or real closed) in a language with function symbols for a (frequently standard, but sometimes nonstandard) family of analytic functions. Results are established for the nonstandard fields using algebraic properties of the ring of "functions" in the language (for example parametrized Weierstrass Preparation and Division, a Strong Noetherian Property and sometimes compactness). In this paper we endeavor to give a sufficiently general framework for fields with analytic structure to unify the above-mentioned work and hopefully to facilitate further applications.

In Section 2 we briefly review some facts about maximally complete valued fields. In Section 3 we give the basic definitions and properties of (ordered) fields with real analytic structure. In Section 4 we give the definitions and basic properties of Henselian fields with separated analytic structure. Basic for model-theoretic results (such as cell decomposition, $o$-minimality) is an understanding of functions of one variable defined in an arbitrary model. The basic results on functions of one variable in the separated case are presented in Section 5. These results also extend the classical affinoid results when $K$ is algebraically closed and complete (and of rank 1) to the nonalgebraically closed and the quasi-affinoid cases. The corresponding results on functions of one variable in the real closed case are in [CLR2, Section 3]. In Section 6 we generalize the cell decompositions of [CLR1] to Henselian fields with analytic structure and also establish "preservation of balls" and a Jacobian property for these structures, which are useful for change of variables formulas for integrals.

Another axiomatic approach to analytic structures, close to the one in [DHM], is given by Scanlon in [Scan2] where he also studies liftings of the Frobenius.

\section{Notation}

For $K$ a valued field, write $K^{\circ}$ for its valuation ring with maximal ideal $K^{\circ \circ}, \widetilde{K}$ its residue field and $K_{\text {alg }}$ for its algebraic closure. Usually $K$ is Henselian and then $K_{\text {alg }}$ carries a unique valuation extending that of $K$. We denote the (multiplicative) norm on $K$ by $|\cdot|$.

\section{Maximally complete fields}

In this section we recall some of the properties of maximally complete valued fields, also called Malcev-Neumann fields. See [Kap], $[\mathrm{Po}]$ and the references therein. In the equicharacteristic case, the maximally complete field $K$ with residue field $\widetilde{K}$ and value group $\Gamma$ (most often written additively, sometimes multiplicatively) is

$$
\widetilde{K}((\Gamma))=\left\{\sum_{g \in I} a_{g} t^{g}: a_{g} \in \widetilde{K}, I \subset \Gamma \text { well ordered }\right\} .
$$

In the mixed characteristic case (see $[\overline{\mathrm{Po}}]$ ) we let $k$ be a perfect field of characteristic $p$, $R$ a complete discrete valuation ring of characteristic zero with $\widetilde{R}=k$ and let $E \subset R$ 
be a set of multiplicative representatives of $k$ in $R$ (i.e. $E$ contains 0 and one element from each nonzero equivalence class of $\widetilde{R}=R / R^{\circ}$ where $R^{\circ}$ is the maximal ideal of $R$ ). Choose an embedding $\mathbb{Z} \hookrightarrow \Gamma$. The maximally complete field of mixed characteristic is constructed in $[\mathrm{Po}]$. It is

$$
R((\Gamma))=\left\{\sum_{g \in I} a_{g} t^{g}: a_{g} \in E, I \subset \Gamma \text { well ordered }\right\} .
$$

(The "coefficients" $a_{g}$ are added and multiplied according to the addition and multiplication in $R$, using the fact that each element of $R$ has a unique representation of the form $\sum_{g \in \mathbb{N}} a_{g} t^{g}, a_{g} \in E$.)

In both the equicharacteristic and the mixed characteristic cases we can write an arbitrary element of $\widetilde{K}((\Gamma))$ or $R((\Gamma))$ as $a=\sum_{g \in \Gamma} a_{g} t^{g}$ where the sum is over all of $\Gamma$, but we require that $I_{a}=\left\{g: a_{g} \neq 0\right\}$ be a well ordered subset of $\Gamma$. We call $I_{a}$ the support of $a$, denoted $\operatorname{supp}(a)$.

The (multiplicative) norm $|\cdot|$ on $R((\Gamma))$ is $\left|\sum_{g \in I} a_{g} t^{g}\right|=t^{g_{0}}$ where $g_{0}$ is the smallest $g \in I$ with $a_{g} \neq 0$.

\section{Real closed fields with analytic structure}

As we mentioned in the introduction, nonarchimedean real closed fields with various analytic structures were considered in [DMM1]-[DMM3], [LR4] and [CLR2]. In this section we establish a general framework for fields with real analytic structure.

\subsection{Definitions}

Let $A_{n, \alpha}, n \in \mathbb{N}, \alpha \in \mathbb{R}, \alpha>0$, be the ring of real power series in $\left(\xi_{1}, \ldots, \xi_{n}\right)$ (i.e. elements of $\left.\mathbb{R}\left[\left[\xi_{1}, \ldots, \xi_{n}\right]\right]\right)$ with radius of convergence $>\alpha$, and let $\Gamma$ be an ordered abelian group.

In analogy with the notation of Section 2 we define

$$
A_{n, \alpha}((\Gamma)):=\left\{\sum_{g \in I} f_{g} t^{g}: f_{g} \in A_{n, \alpha} \text { and } I \subset \Gamma \text { well ordered }\right\} .
$$

Clearly $A_{0, \alpha}((\Gamma))=\mathbb{R}((\Gamma)) \subset A_{n, \alpha}((\Gamma))$. Note that $\mathbb{R}((\Gamma))$ inherits a natural order from the order on $\mathbb{R}$ and on $\Gamma$. If $\Gamma$ is divisible, then $\mathbb{R}((\Gamma))$ is real closed. We shall denote the absolute value arising from this order by $|\cdot|$, and denote the norm on $\mathbb{R}((\Gamma))$ by $\|\cdot\|$ to distinguish it from $|\cdot|$. This norm extends to the Gauss norm on $A_{n, \alpha}((\Gamma))$, also denoted $\|\cdot\|$, defined by $\left\|\sum_{g \in I} f_{g} t^{g}\right\|=t^{g_{0}}$ where $g_{0}$ is the smallest $g \in I$ with $f_{g} \neq 0$. We call $f_{g_{0}}$ the top slice of $f$, and call $f$ regular in $\xi_{n}$ of degree $s$ at a point $c$ in $[-\alpha, \alpha]^{n}$ if $f_{0}$ is regular in the classical sense in $\xi_{n}$ of degree $s$ at the point $c^{\circ}$, the element of $\mathbb{R}^{n}$ closest to $c$. 
Definition 3.1.1 (Real Weierstrass system). Let $\mathcal{B}=\left\{B_{n, \alpha}: n \in \mathbb{N}, \alpha \in \mathbb{R}_{+}\right\}$be a family of $\mathbb{R}$-algebras satisfying

$$
\mathbb{R}\left[\xi_{1}, \ldots, \xi_{n}\right] \subset B_{n, \alpha} \subset A_{n, \alpha}((\Gamma))
$$

for each $n$ and $\alpha$. We call the family $\mathcal{B}$ a real Weierstrass system if the following conditions (a) and (b) are satisfied:

(a) (i) If $m \leq m^{\prime}$ and $\alpha^{\prime} \leq \alpha$ then $B_{m, \alpha} \subset B_{m^{\prime}, \alpha^{\prime}}$ and $B_{0, \alpha}=B_{0, \alpha^{\prime}}$. (We allow the possibility that $B_{0}:=B_{0, \alpha}$ is a proper $\mathbb{R}$-subalgebra of $\mathbb{R}((\Gamma))$.)

(ii) If $f \in B_{m+n, \alpha}$ and $f=\sum_{\mu} \bar{f}_{\mu}\left(\xi_{1}, \ldots, \xi_{m}\right) \eta^{\mu}$ where $\eta=\left(\xi_{m+1}, \ldots, \xi_{m+n}\right)$, then the $\bar{f}_{\mu}$ are in $B_{m, \alpha}$.

(iii) If $f \in B_{m, \alpha}, a \in(-\alpha, \alpha)^{n} \cap \mathbb{R}^{n}$, and $r \in \mathbb{R}_{+}$, then $f(r \cdot(\xi+a))$ belongs to $B_{m, \delta}$ with $\delta:=\min ((\alpha-a) / r,(\alpha+a) / r)$, where $f(r \cdot(\xi+a))$ is considered naturally as an element of $A_{n, \delta}((\Gamma))$.

(iv) If $f \in B_{n, \alpha}$ then $a f \in B_{n, \alpha}$ for some $a$ in $B_{0}$ satisfying $\|a f\|=1$ with $\|\cdot\|$ the Gauss norm on $A_{n, \alpha}((\Gamma))$.

(b) Weierstrass Division: If $f \in B_{n, \alpha}$ with $\|f\|=1$ is regular in $\xi_{n}$ of degree $s$ at 0 , there is a $\delta \in \mathbb{R}, \delta>0$, such that if $g \in B_{n, \alpha}$, then there are unique $Q \in B_{n, \delta}$ and $R_{0}\left(\xi^{\prime}\right), \ldots, R_{s-1}\left(\xi^{\prime}\right) \in B_{n-1, \delta}$, with $\|Q\|,\left\|R_{i}\right\| \leq\|g\|$, such that

$$
g=Q f+R_{0}\left(\xi^{\prime}\right)+R_{1}\left(\xi^{\prime}\right) \xi_{n}+\cdots+R_{s-1}\left(\xi^{\prime}\right) \xi_{n}^{s-1} .
$$

Definition 3.1.2. If a real Weierstrass system $\mathcal{B}:=\left\{B_{n, \alpha}: n \in \mathbb{N}, \alpha \in \mathbb{R}_{+}\right\}$satisfies in addition the following condition (c), then call $\mathcal{B}$ a strong real Weierstrass system.

(c) If $f\left(\xi, \eta_{1}, \eta_{2}\right) \in B_{n+2, \alpha}$ there are $f_{1}\left(\xi, \eta_{1}, \eta_{3}\right), f_{2}\left(\xi, \eta_{2}, \eta_{3}\right)$ and $Q\left(\xi, \eta_{1}, \eta_{2}, \eta_{3}\right) \in$ $B_{n+3, \alpha}$ such that

$$
f\left(\xi, \eta_{1}, \eta_{2}\right)=f_{1}\left(\xi, \eta_{1}, \eta_{3}\right)+\eta_{2} f_{2}\left(\xi, \eta_{2}, \eta_{3}\right)+Q \cdot\left(\eta_{1} \eta_{2}-\eta_{3}\right) .
$$

Remark 3.1.3 (Weierstrass Preparation). Suppose that the family $\mathcal{B}=\left\{B_{n, \alpha}\right\}$ is a real Weierstrass system. If $f \in B_{n, \alpha}$ with $\|f\|=1$ is regular in $\xi_{n}$ of degree $s$ at 0 , then there is a $\delta \in \mathbb{R}, \delta>0$, such that we can write uniquely

$$
f=\left[\xi_{n}^{s}+A_{1}\left(\xi^{\prime}\right) \xi_{n}^{s-1}+\cdots+A_{s}\left(\xi^{\prime}\right)\right] U(\xi)
$$

where:

$$
\begin{aligned}
& A_{1}, \ldots, A_{s}, \in B_{n-1, \delta}, \text { and } U \in B_{n, \delta} \text { is a unit, } \\
& \left\|A_{1}\right\|, \ldots,\left\|A_{s}\right\|,\|U\| \leq 1, \\
& \left\|A_{1}(0)\right\|, \ldots,\left\|A_{s}(0)\right\|<1 \text { and }\|U(0)\|=1 .
\end{aligned}
$$

This can be seen by taking $g=\xi_{n}^{s}$ in axiom (b) and using the case $s=0$ to see that if $\|Q\|=1$ and $Q$ is regular of degree 0 at 0 , then $Q$ is a unit. (The special case $n=s=0$ shows that if $a \in B_{0},\|a\|=1$, then $a$ is a unit in $B_{0}$, and hence, using (a)(iv), that $B_{0}$ is a subfield of $\mathbb{R}((\Gamma))$.) 
Remark 3.1.4. (i) Condition (c) of Definition 3.1.2 is used in the proof of $o$-minimality (see Theorem 3.4.3). This condition does not follow from the other conditions, as can be seen in Example 3.3 (7) below. However, every real Weierstrass system can be extended to a strong real Weierstrass system since the Weierstrass system $\left\{A_{n, \alpha}((\Gamma))\right\}$ is strong (see Example 3.3.6)). To prove $o$-minimality for real closed fields with (not necessarily strong) analytic structure it is sufficient to prove $o$-minimality for real closed fields with strong analytic structure. The proof of $o$-minimality for real closed fields with strong analytic structure is given in [CLR2, Section 3], where condition (c) is used (implicitly), roughly speaking to write $f(x, 1 / x)=g(x)+(1 / x) h(1 / x)$.

(ii) Weierstrass Division guarantees that if $f \in B_{n, \alpha}$ and $\xi_{i}$ divides $f$ as an element of $A((\Gamma))$ (i.e. $\left.f\left(\xi_{1}, \ldots, \xi_{i-1}, 0, \xi_{i+1}, \ldots, \xi_{n}\right)=0\right)$ then there is a $\delta>0$ and an element $g \in B_{n, \delta}$ such that $f=\xi_{i} g$. It is not the case that if $f \in A_{n, \alpha}$, has no zero in the (real) polydisc $[-\alpha, \alpha]^{n}$, then $f$ is a unit in $B_{n, \alpha}$. To see this consider $f=1+x^{2} \in B_{1,1}$. The units in $B_{n, \alpha}$ are the functions that have no zeros in the complex polydisc $\{x \in \mathbb{C}$ : $|x| \leq \beta\}^{n}$ for some $\alpha<\beta \in \mathbb{R}$. However, if $f \in B_{n, \alpha}$ and $f_{0}$, the top slice of $f$ has no zero in $[-\alpha, \alpha]^{n}$ then $[-\alpha, \alpha]^{n}$ can be covered by finitely many (smaller) polydiscs on each of which $f_{0}$ is a unit. It then follows from conditions (a) and (b) of Definition 3.1.1 that $f$ is a unit in the rings $B_{n, \delta}$ on each of these smaller polydiscs. If $f \in B_{n, \alpha}$ and $f_{0}$ is not 0 at 0 , then $f$ is regular of degree 0 at 0 , and hence by Weierstrass Division, a unit. In other words, if $f \in B_{n, \alpha}$ satisfies $f_{0}(0) \neq 0$ then $f$ is a unit in $B_{n, \delta}$ for some $\delta \in \mathbb{R}_{+}$.

(iii) It follows from condition (a)(ii) of Definition 3.1.1 that if $f \in B_{m+n, \alpha}$ and $f=$ $\sum_{\mu} \bar{f}_{\mu}\left(\xi_{1}, \ldots, \xi_{m}\right) \eta^{\mu}$, then

$$
\sum_{\substack{\mu_{i}>d \text { for } \\ i=1, \ldots, n}} \bar{f}_{\mu}\left(\xi_{1}, \ldots, \xi_{m}\right) \eta^{\mu} \in B_{m+n, \alpha}
$$

(iv) The proofs in [CLR2, Section 2] (in particular of Theorem 2.5), with very minor modifications, show that the rings $B_{n, \alpha}=A_{n, \alpha}((\Gamma))$ satisfy conditions (a) and (b) of Definition 3.1.1 Condition (c) is immediate. We shall refer to this Weierstrass System as $\mathcal{A}((\Gamma))$ and call it the full Weierstrass system based on $\Gamma$. It is the largest Weierstrass system corresponding to $\Gamma$. The axioms allow us to "evaluate" elements of $A_{n, \alpha}((\Gamma))$ at arguments $a \in B_{0}^{n} \cap[-\alpha, \alpha]^{n}$. By axiom (a)(iii) it is sufficient to consider the case $a^{\circ}=0$, i.e. $a=\left(a_{1}, \ldots, a_{n}\right)$ with the $a_{i}$ all infinitesimal (i.e. $\left.\left\|a_{i}\right\|<1\right)$. Then, by Weierstrass Division,

$$
f=b+\sum_{i} Q_{i} \cdot\left(\xi_{i}-a_{i}\right)
$$

for a unique $b \in \mathbb{R}((\Gamma))$, since $\xi_{i}-a_{i}$ is regular of degree one in $\xi_{i}$ at 0 . Let $J \subset \Gamma$ be well ordered and satisfy $J+J=J$ and $\operatorname{supp}(f), \operatorname{supp}\left(a_{i}\right) \subset J$. Then an induction on $J$ shows that $b=\sum_{g \in I} f_{g}(a) t^{g}$, where $f_{g}(\xi)=\sum_{\nu} a_{g \nu} \xi^{\nu}$ and $f_{g}(a)=\sum_{\nu} a_{g \nu} a^{\nu}$. A similar argument allows us to "compose" elements of $A((\Gamma))$. More precisely, if $f \in B_{m, \alpha}$ and $g_{1}, \ldots, g_{m} \in B_{n, \gamma}$ with $\left(g_{i}\right)_{0}(0)=a_{i}$, where $\left(g_{i}\right)_{0}$ is the top slice of $g_{i},\left|a_{i}\right|<\alpha$, and $\left\|g_{i}\right\| \leq 1$ for $i=1, \ldots, m$, then there is a $\beta \in \mathbb{R}, \beta>0$, such that $f\left(g_{1}(\xi), \ldots, g_{m}(\xi)\right)$ is in $B_{m, \beta}$. 
(v) By (iii), $A((\Gamma))$ is also closed under the taking of natural derivatives in $A((\Gamma))$. More precisely, taking $m=1$ for notational convenience, composing $f \in B_{1, \alpha}$ with $x \mapsto x+y$, writing $f(x+y)=f_{0}(x)+f_{1}(x) y+\cdots$ and defining $f^{\prime}$ as $f_{1}$, by construction one sees that $f^{\prime}$ is in $B_{1, \delta}$ for some $0<\delta \leq \alpha$, giving a derivation $B_{1, \delta} \rightarrow B_{1, \delta}$ which extends the natural derivation on $A_{1, \delta}$.

(vi) Since all the above mentioned data are unique in $\mathcal{A}((\Gamma))$, and by the axioms exist in any Weierstrass system $\mathcal{B}$ contained in $\mathcal{A}((\Gamma))$, they are also unique in $\mathcal{B}$.

\subsection{The Strong Noetherian Property}

We prove a Strong Noetherian Property (Theorem 3.2.2) for real Weierstrass systems, closely following [CLR2, Lemma 2.9 and Theorem 2.10]. Since this is a fundamental property, and the ideas of the proof are used again in Section 4.2, we give complete proofs. We write $B_{m, \alpha}^{\circ}:=\left\{f \in B_{m, \alpha}:\|f\| \leq 1\right\}$.

The following lemma is used to prove Theorem 3.2 .2 .

Lemma 3.2.1. Let $\mathcal{B}=\left\{B_{m, \alpha}\right\}$ be a real Weierstrass system and let $f(\xi, \eta)=$ $\sum_{\mu} \bar{f}_{\mu}(\xi) \eta^{\mu} \in B_{m+n, \alpha}$. Then the $\bar{f}_{\mu}$ are in $B_{m, \alpha}$, by Definition 3.1.1 (a)(ii). There is an integer $d \in \mathbb{N}$, a constant $\beta \in \mathbb{R}, 0<\beta \leq \alpha$, and $g_{\mu} \in B_{m+n, \beta}^{\circ}$ for $|\mu|<d$, such that, considering $f \in B_{m+n, \beta}$,

$$
f=\sum_{|\mu|<d} \bar{f}_{\mu}(\xi) g_{\mu}(\xi, \eta)
$$

Proof. By property (a)(iv) of Definition 3.1.1, we may assume that $\|f\|=1$. Choose a $v_{0}$ such that $\left\|\bar{f}_{\nu_{0}}\right\|=1$. Making an $\mathbb{R}$-linear change of variables (allowed by Remark 3.1.4), and shrinking $\alpha$ if necessary, we may assume that $\bar{f}_{\nu_{0}}$ is regular in $\xi_{m}$ at 0 of degree $s$, say. Write $\xi^{\prime}$ for $\left(\xi_{1}, \ldots, \xi_{m-1}\right)$. By Weierstrass Division there is a $\beta>0$ and there are $Q(\xi, \eta) \in B_{m+n, \beta}$ and

$$
R(\xi, \eta)=R_{0}\left(\xi^{\prime}, \eta\right)+\cdots+R_{s-1}\left(\xi^{\prime}, \eta\right) \xi_{m}^{s-1} \in B_{m+n-1, \beta}\left[\xi_{m}\right]
$$

such that

$$
f(\xi, \eta)=\bar{f}_{\nu_{0}}(\xi) Q(\xi, \eta)+R(\xi, \eta) .
$$

By induction on $m$, we may write

$$
R_{0}=\sum_{|\mu|<d} \bar{R}_{0 \mu}\left(\xi^{\prime}\right) g_{\mu}\left(\xi^{\prime}, \eta\right)
$$

for some $d \in \mathbb{N}$, some $\beta>0$ and $g_{\mu}\left(\xi^{\prime}, \eta\right) \in B_{m+n-1, \beta}^{\circ}$. Writing $R=\sum_{v} \bar{R}_{\nu}(\xi) \eta^{\nu}$, observe that each $\bar{R}_{\nu}$ is a $B_{m, \beta}^{\circ}$-linear combination of the $\bar{f}_{\nu}$, since, taking the coefficient of $\eta^{\nu}$ on both sides of the equation $f(\xi, \eta)=\bar{f}_{v_{0}}(\xi) Q(\xi, \eta)+R(\xi, \eta)$, we have

$$
\bar{f}_{v}=\bar{f}_{v_{0}} \bar{Q}_{v}+\bar{R}_{v}
$$


Consider

$$
\begin{aligned}
f-\bar{f}_{v_{0}} Q-\sum_{|\mu|<d} \bar{R}_{\mu}(\xi) g_{\mu}\left(\xi^{\prime}, \eta\right) & =: S_{1} \xi_{m}+S_{2} \xi_{m}^{2}+\cdots+S_{s-1} \xi_{m}^{s-1} \\
& =\xi_{m}\left[S_{1}+S_{2} \xi_{m}+\cdots+S_{s-1} \xi_{m}^{s-2}\right] \\
& =: \xi_{m} \cdot S, \text { say, }
\end{aligned}
$$

where the $S_{i}$ are in $B_{m+n-1, \beta}^{\circ}$. Again, observe that each $\bar{S}_{v}$ is a $B_{m, \beta}^{\circ}$-linear combination of the $\bar{f}_{v^{\prime}}$. Complete the proof by induction on $s$, working with $S$ instead of $f$.

Theorem 3.2.2 (Strong Noetherian Property). Let $\mathcal{B}=\left\{B_{m, \alpha}\right\}$ be a real Weierstrass system and let $f(\xi, \eta)=\sum_{\mu} \bar{f}_{\mu}(\xi) \eta^{\mu} \in B_{m+n, \alpha}$. Then the $\bar{f}_{\mu}$ are in $B_{m, \alpha}$ and there is an integer $d \in \mathbb{N}$, a constant $\beta \in \mathbb{R}, \beta>0$, and units $U_{\mu}(\xi, \eta) \in B_{m+n, \beta}^{\circ}$ for $|\mu| \leq d$, such that, considering $f \in B_{m+n, \delta}$,

$$
f=\sum_{\mu \in J} \bar{f}_{\mu}(\xi) \eta^{\mu} U_{\mu}(\xi, \eta),
$$

where $J$ is a subset of $\{0,1, \ldots, d\}^{n}$.

Proof. It is sufficient to show that there are an integer $d$, a set $J \subset\{0,1, \ldots, d\}^{n}$, and $g_{\mu} \in B_{m+n, \beta}^{\circ}$ such that

$$
f=\sum_{\mu \in J} \bar{f}_{\mu}(\xi) \eta^{\mu} g_{\mu}(\xi, \eta)
$$

since then, rearranging the sum if necessary, we may assume that each $g_{\mu}$ is of the form $1+h_{\mu}$ where $h_{\mu} \in(\eta) B_{m+n, \beta}^{\circ}$. Shrinking $\beta$ if necessary will guarantee that the $g_{\mu}$ are units. But then it is in fact sufficient to prove (3.1) for $f$ replaced by

$$
f_{I_{i}}:=\sum_{\mu \in I_{i}} \bar{f}_{\mu}(\xi) \eta^{\mu}
$$

for each $I_{i}$ in a finite partition $\left\{I_{i}\right\}$ of $\mathbb{N}^{n}$ and to show that $f_{I_{i}}$ is in $B_{m+n, \beta}$.

By Lemma 3.2.1 there is an integer $d \in \mathbb{N}$, a constant $\beta \in \mathbb{R}, 0<\beta \leq \alpha$, and $g_{\mu} \in B_{m+n, \beta}^{\circ}$ for $|\mu| \leq d$ such that

$$
f=\sum_{|\mu| \leq d} \bar{f}_{\mu}(\xi) g_{\mu}(\xi, \eta) .
$$

Rearranging, we may assume for $\nu, \mu \in\{1, \ldots, d\}^{n}$ that $\left(\overline{g_{\mu}}\right)_{\nu}$ equals 1 if $\mu=\nu$, and 0 otherwise.

Focus on $f_{I_{1}}(\xi, \eta)$, defined as above by

$$
f_{I_{1}}(\xi, \eta)=\sum_{\mu \in I_{1}} \bar{f}_{\mu}(\xi) \eta^{\mu}
$$

with

$$
I_{1}:=\{1, \ldots, d\}^{n} \cup\left\{\mu: \mu_{i} \geq d \text { for all } i\right\}
$$


and note that

$$
f_{I_{1}}(\xi, \eta)=\sum_{|\mu| \leq d} \bar{f}_{\mu}(\xi) g_{\mu, I_{1}}(\xi, \eta)
$$

with $g_{\mu, I_{1}}(\xi, \eta) \in B_{m+n, \beta}^{\circ}$ defined by the corresponding sum

$$
g_{\mu, I_{1}}(\xi, \eta)=\sum_{\nu \in I_{1}} \bar{g}_{\mu, \nu}(\xi) \eta^{\nu}
$$

The $g_{\mu, I_{1}}$ and $f_{I_{1}}$ are in $B_{m+n, \beta}^{\circ}$. This follows from the axioms and induction on $n$. (See Remark 3.1.4)

It is now clear that $g_{\mu, I_{1}}$ is of the form $\eta^{\mu}\left(1+h_{\mu}\right)$ where $h_{\mu} \in(\eta) B_{m+n, \beta}^{\circ}$.

One now completes the proof by noting that $f-f_{I_{1}}$ is a finite sum of terms of the form $f_{I_{j}}$ for $j>1$ and $\left\{I_{i}\right\}_{i}$ a finite partition of $\mathbb{N}^{n}$ and where each $f_{I_{j}}$ for $j>1$ is in $B_{m+n, \beta}^{\circ}$ and is of the form $\eta_{i}^{\ell} q\left(\xi, \eta^{\prime}\right)$ where $\eta^{\prime}$ is $\left(\eta_{1}, \ldots, \eta_{i-1}, \eta_{i+1}, \ldots, \eta_{n}\right)$ and $q$ is in $\mathcal{R}_{m+n-1, \beta}^{\circ}$. These terms can be handled by induction on $n$.

Definition 3.2.3. Let $\mathcal{B}=\left\{B_{n, \alpha}\right\}$ be a real Weierstrass system. Let $K$ be an ordered field containing $B_{0}$ as an ordered subfield. For each $n \in \mathbb{N}, \alpha \in \mathbb{R}, \alpha>1$ let $\sigma_{n, \alpha}$ be an $\mathbb{R}$-algebra homomorphism from $B_{n, \alpha}$ to the ring of $K$-valued functions on $[-1,1]^{n}$, compatible with the inclusions $B_{n, \alpha} \subset B_{n, \beta}$ for $\beta<\alpha$, and respecting the translation conditions of Definition 3.1.1 a)(iii). Write $\sigma_{n}$ for the induced homomorphism on $\bigcup_{\alpha>1} B_{n, \alpha}$. Suppose that the maps $\sigma_{n}$ satisfy

(i) $\sigma_{0}$ is the inclusion $B_{0} \subset K$,

(ii) $\sigma_{m}\left(\xi_{i}\right)$ is the $i$-th coordinate function on $\left(K^{\circ}\right)^{m}, i=1, \ldots, m$,

(iii) $\sigma_{m+1}$ extends $\sigma_{m}$, where we identify in the obvious way functions on $\left(K^{\circ}\right)^{m}$ with functions on $\left(K^{\circ}\right)^{m+1}$ that do not depend on the last coordinate.

Then we call the family $\sigma:=\left\{\sigma_{n, \alpha}\right\}$ a real analytic $\mathcal{B}$-structure on $K$.

\subsection{Examples of real analytic structures}

(1) Take $\Gamma=\{0\}$ and $B_{n, \alpha}=A_{n, \alpha}((\{0\}))=A_{n, \alpha}$. Then by the observations in [DMM1] every complete or maximally complete real closed valued field containing $\mathbb{R}$ has analytic $\mathcal{B}$-structure. In particular, $\mathbb{R}$ has analytic $\mathcal{B}$-structure, so $\mathbb{R}$ with the subanalytic structure studied in $[\mathrm{DD}]$ is covered by our definitions.

(2) Take $\Gamma=\mathbb{Q}$ and for all $\alpha>0$ let

$$
\begin{aligned}
B_{n, \alpha} & :=B_{n}:=\left\{\sum_{\gamma_{i} \in \mathbb{Q}} t^{\gamma_{i}} p_{i}(\xi): p_{i}(\xi) \in \mathbb{R}[\xi] \text { and } \gamma_{i}-\varepsilon \operatorname{deg}\left(p_{i}\right) \rightarrow \infty \text { for some } \varepsilon>0\right\} \\
& \subsetneq A_{n, \alpha}((\mathbb{Q}))
\end{aligned}
$$

(in other words, the $t$-adically overconvergent power series, $K\langle\langle\xi\rangle\rangle$, where $K$ is the completion of the field of real Puiseux series). Then we are in the context of considering the field $K$ with the rings of $t$-adically overconvergent power series. This is the case considered in [LR4]. Observe that in this example $\mathbb{R}((\Gamma)) \not \subset K$. 
(3) If in the context of (2) we take $\mathcal{B}^{\prime}=\left\{B_{n, \alpha}^{\prime}\right\}$, with

$$
\begin{aligned}
& B_{n, \alpha}^{\prime}:=B_{n}^{\prime} \\
& :=\left\{\sum_{\gamma_{i} \in \mathbb{Z}[1 / m]} t^{\gamma_{i}} p_{i}(\xi): p_{i}(\xi) \in \mathbb{R}[\xi] \text { and } \gamma_{i}-\varepsilon \operatorname{deg}\left(p_{i}\right) \rightarrow \infty \text { for some } \varepsilon>0, m \in \mathbb{N}\right\}
\end{aligned}
$$

we may consider the field of real Puiseux series (rather than its completion) with real analytic $\mathcal{B}^{\prime}$-structure. Hence this is an example of a field with real analytic structure that is not complete.

(4) Take $\Gamma=\mathbb{Q}$ and let

$$
B_{n, \alpha}=\left\{\sum t^{\gamma_{i}} f_{\gamma_{i}}(\xi): f_{\gamma_{i}} \in A_{n, \alpha}, \mathbb{Q} \ni \gamma_{i} \rightarrow \infty\right\} \subsetneq A_{n, \alpha}((\mathbb{Q})) .
$$

Then we are in the context of Section 2 of [CLR2] where we considered the completion of the field of Puiseux series with real analytic $\mathcal{B}$-structure. If we take

$$
B_{n, \alpha}^{\prime}=\left\{\sum_{\gamma_{i} \in \mathbb{Z}[1 / m]} t^{\gamma_{i}} f_{\gamma_{i}}(\xi): f_{\gamma_{i}} \in A_{n, \alpha}, \gamma_{i} \rightarrow \infty \text {, for some } m \in \mathbb{N}\right\}
$$

and $\mathcal{B}^{\prime}:=\left\{B_{n, \alpha}^{\prime}\right\}$, then the field of real Puiseux series has analytic $\mathcal{B}^{\prime}$-structure.

(5) Take $\Gamma=\mathbb{Q}^{n}$ with $\mathbb{Q}^{n}$ ordered lexicographically, and

$$
B_{n, \alpha}=\left\{\sum_{g \in I} t^{g} f_{g}(\xi): f_{g} \in A_{n, \alpha}, I \subset \mathbb{Q}^{n} \text { well ordered }\right\} \text {. }
$$

Then we are in the context of Section 4 of [CLR2]. Note that even for $n=1$ there are more functions in this analytic structure than in the one of example (4).

(6) (Cf. Remark 3.1.4) Take $\Gamma$ arbitrary and $B_{n, \alpha}=A_{n, \alpha}((\Gamma))$. Then $\mathcal{A}((\Gamma)):=$ $\left\{B_{n, \alpha}\right\}$ is a strong real Weierstrass system. The proofs of [CLR2] Section 2], with very minor modifications, show that this family is a strong real Weierstrass system and that $\mathbb{R}((\Gamma))$ has real analytic $\mathcal{A}((\Gamma))$-structure. We call this the full real analytic $\mathbb{R}((\Gamma))$ structure.

(7) If we take $\Gamma=\{0\}$ and $B_{n, \alpha}$ the ring of algebraic power series with radius of convergence $>\alpha$, then the $B_{n, \alpha}$ satisfy conditions (a) and (b) of Definition 3.1.1. but not condition (c). Indeed, if $f=\sum a_{i j} \eta_{1}^{i} \eta_{2}^{j}$ and $f_{1}$ and $f_{2}$ are as in condition (c), then $f_{1}\left(0, \eta_{3}\right)=\sum a_{i i} \eta_{3}^{i}$ is the "diagonal" of $f$. Take

$$
f=\left(1-4 \eta_{1}\right)^{-1 / 2}\left(1-4 \eta_{2}\right)^{-1 / 2} \in A_{2, \alpha}
$$

for $\alpha<1 / 4$. Then $f$ is algebraic, but the diagonal of $f$ is $\sum_{i}\left(\begin{array}{c}2 i \\ i\end{array}\right)^{2} \eta_{3}^{i}$, which is the elliptic integral $\frac{2}{\pi} \int_{0}^{\pi / 2} \frac{d t}{\sqrt{1-16 \eta_{3} \sin ^{2} t}}$ and not algebraic. (Cf. [DL2].) 


\subsection{Model-theoretic results}

Definition 3.4.1. Let $\mathcal{B}:=\left\{B_{n, \alpha}\right\}$ be a real Weierstrass system. Define the language $\mathcal{L}_{\mathcal{B}}$ to be the language of ordered fields, $\left\langle+,-, \cdot,^{-1},<\right\rangle$, together with symbols for all elements of $B_{n, \alpha}$ for all $n$ and $\alpha>1$.

Remark 3.4.2. Let $\mathcal{B}:=\left\{B_{n, \alpha}\right\}$ be a real Weierstrass system and $\sigma$ a real analytic $\mathcal{B}$ structure on a field $K$. Then for each $\alpha$ with $1 \geq \alpha>0$, and each $f \in B_{n, \alpha}, f$ yields a unique definable function (given by a term) from, for example, $[-\alpha / 2, \alpha / 2]$ to $K$, by rescaling as in axiom (a).

Theorem 3.4.3 (Quantifier Elimination and $o$-minimality). Let $K$ be a real closed field with a real analytic $\mathcal{B}$-structure for some real Weierstrass system $\mathcal{B}$. Then $K$ has quantifier elimination in the language $\mathcal{L}_{\mathcal{B}}$ (with the natural interpretation) and is o-minimal in $\mathcal{L}_{\mathcal{B}}$.

Proof. The proof of the quantifier elimination for $\mathcal{L}_{\mathcal{B}}$ is a minor modification of the last section of [DD]; see [DMM1], [CLR2], [LR4] for similar such modifications. By this quantifier elimination, the natural theory of real closed fields with real analytic $\mathcal{B}$ structure is complete in the language $\mathcal{L}_{\mathcal{B}}$. Hence, it is enough to prove $o$-minimality for an expansion of one model. We prove $o$-minimality of $K:=\mathbb{R}((G))$ in the language $\mathcal{L}_{\mathcal{B}^{\prime}}$ with $G$ the divisible closure of $\Gamma$ and where we take $B_{n, \alpha}^{\prime}:=A_{n, \alpha}((G))$ to form our real Weierstrass system $\mathcal{B}^{\prime}$ (cf. Example 3.3 6)). Since $B_{n, \alpha} \subset B_{n, \alpha}^{\prime}$ for each $n, \alpha$, it is enough to prove that $K$ is $o$-minimal in $\mathcal{L}_{\mathcal{B}^{\prime}}$. Now one can analyze definable functions in one variable (using annuli and the fact that $\mathcal{B}^{\prime}$ is strong) exactly as in [CLR2, Section 3], yielding $o$-minimality as in [CLR2]. The strongness assumption (Definition 3.1.2] is used implicitly in [CLR2, Section 3]. (This analysis is similar to, but simpler than the one given in Section 5 below for the separated case, since one is much closer to the algebraically closed case.)

\section{Henselian fields with analytic structure}

In this section we present a general theory of (Henselian) fields with analytic structure, generalizing the presentations in [vdD], [DHM], [LR3] and [CLR1]. We distinguish between separated analytic structures and strictly convergent analytic structures. In Section 4.1 we present the definitions of separated Weierstrass systems and Henselian fields with separated analytic structure. In Section 4.2 we discuss the strong Noetherian property for separated Weierstrass systems, and in Section 4.3 we define strictly convergent Weierstrass systems and fields with strictly convergent analytic structure. In Section 4.4 we give examples of Henselian fields with analytic structure. In Section 4.5 we develop properties of Henselian fields with analytic structure. In Section 5 we analyze one variable terms (functions) of the theory of Henselian fields with separated analytic structure.

In this whole section, let $A$ be a commutative ring with unit with a fixed proper ideal $I$

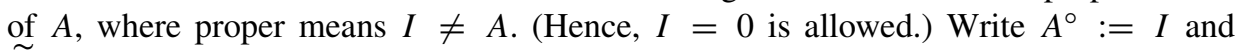
$\widetilde{A}:=A / I$. 


\subsection{Henselian fields with separated analytic structure}

We consider polynomial rings and power series rings in two kinds of variables, written $\xi_{i}$ and $\rho_{j}$, which play different roles. Roughly speaking, we think of the $\xi_{i}$ as varying over the valuation ring (or the closed unit disc) $K^{\circ}$ of a valued field $K$, and the variables $\rho_{j}$ as varying over the maximal ideal (or the open unit disc) $K^{\circ \circ}$ of $K^{\circ}$. We use the terminology "separated" in analogy to the rings of separated power series whose theory was developed in [LL1], [B]], [LL2], and especially [LR1]. Let $A$ and $I \varsubsetneqq A$ be as at the beginning of Section 4

A first instance where these variables play different roles is:

Definition 4.1.1 (Regular). Let $f$ be a power series in $A\left[\left[\xi_{1}, \ldots, \xi_{m}, \rho_{1}, \ldots, \rho_{n}\right]\right]$, and let $J$ be the ideal

$$
J:=\left\{\sum_{\mu, \nu} a_{\mu, \nu} \xi^{\mu} \rho^{\nu} \in A[[\xi, \rho]]: a_{\mu, \nu} \in I\right\}
$$

of $A[[\xi, \rho]]$, where $A$ and $I$ are as at the beginning of Section 4

(i) $f$ is called regular in $\xi_{m}$ of degree $d$ when $f$ is congruent in $A[[\xi, \rho]]$ to a monic polynomial in $\xi_{m}$ of degree $d$ modulo the ideal $B_{1} \subset A[[\xi, \rho]]$, where

$$
B_{1}:=J+(\rho) A[[\xi, \rho]] .
$$

(ii) $f$ is called regular in $\rho_{n}$ of degree $d$ when $f$ is congruent in $A[[\xi, \rho]]$ to $\rho_{n}^{d}$ modulo the ideal $B_{2} \subset A[[\xi, \rho]]$, where

$$
B_{2}:=J+\left(\rho_{1}, \ldots, \rho_{n-1}, \rho_{n}^{d+1}\right) A[[\xi, \rho]] .
$$

Definition 4.1.2 $\left((A, I)\right.$-system). Let $m \leq m^{\prime}$ and $n \leq n^{\prime}$ be natural numbers, and $\xi=\left(\xi_{1}, \ldots, \xi_{m}\right), \xi^{\prime}=\left(\xi_{1}, \ldots, \xi_{m^{\prime}}\right), \xi^{\prime \prime}=\left(\xi_{m+1}, \ldots, \xi_{m^{\prime}}\right), \rho=\left(\rho_{1}, \ldots, \rho_{n}\right), \rho^{\prime}=$ $\left(\rho_{1}, \ldots, \rho_{n^{\prime}}\right)$, and $\rho^{\prime \prime}=\left(\rho_{n+1}, \ldots, \rho_{n^{\prime}}\right)$ be variables. A system $\mathcal{A}=\left\{A_{m, n}\right\}_{m, n \in \mathbb{N}}$ of $A$-algebras $A_{m, n}$ satisfying, for all $m \leq m^{\prime}$ and $n \leq n^{\prime}$,

(i) $A_{0,0}=A$,

(ii) $A_{m, n} \subset A[[\xi, \rho]]$,

(iii) $A_{m, n}\left[\xi^{\prime \prime}, \rho^{\prime \prime}\right] \subset A_{m^{\prime}, n^{\prime}}$,

(iv) the image $\left(A_{m, n}\right)^{\sim}$ of $A_{m, n}$ under the residue map $\sim: A[[\xi, \rho]] \rightarrow \widetilde{A}[[\xi, \rho]]$ is a subring of $\widetilde{A}[\xi][[\rho]]$,

(v) if $f \in A_{m^{\prime}, n^{\prime}}$, say $f=\sum_{\mu, \nu} \bar{f}_{\mu \nu}(\xi, \rho)\left(\xi^{\prime \prime}\right)^{\mu}\left(\rho^{\prime \prime}\right)^{\nu}$, then the $\bar{f}_{\mu \nu}$ are in $A_{m, n}$,

is called a separated $(A, I)$-system.

Definition 4.1.3 (Pre-Weierstrass system). Let $\mathcal{A}=\left\{A_{m, n}\right\}_{m, n \in \mathbb{N}}$ be a separated $(A, I)$ system. Then $\mathcal{A}$ is called a separated pre-Weierstrass system when the two usual Weierstrass Division Theorems hold in the $A_{m, n}$, namely, for $f, g \in A_{m, n}$ :

(a) If $f$ is regular in $\xi_{m}$ of degree $d$, then there exist uniquely determined elements $q \in$ $A_{m, n}$ and $r \in A_{m-1, n}\left[\xi_{m}\right]$ of degree at most $d-1$ such that $g=q f+r$. 
(b) If $f$ is regular in $\rho_{n}$ of degree $d$, then there exist uniquely determined elements $q \in$ $A_{m, n}$ and $r \in A_{m, n-1}\left[\rho_{n}\right]$ of degree at most $d-1$ such that $g=q f+r$.

Sometimes $\mathcal{A}$ is said to be $\operatorname{over}(A, I)$ to specify that $\mathcal{A}$ is a separated $(A, I)$-system.

In fact, since we allow $A$ to be quite general, we need to be able to work locally, using rings of fractions:

Definition 4.1.4 (Rings of fractions). Let $\mathcal{A}=\left\{A_{m, n}\right\}_{m, n \in \mathbb{N}}$ be a separated $(A, I)$ system. Inductively define the concept that an $A$-algebra $C$ is a ring of $\mathcal{A}$-fractions with proper ideal $C^{\circ}$ and with rings $C_{m, n}$ of separated power series over $C$ by

(i) The ring $A$ is a ring of $\mathcal{A}$-fractions with ideal $A^{\circ}=I$ and with rings of separated power series the $A_{m, n}$ from the system $\mathcal{A}$.

(ii) If $B$ is a ring of $\mathcal{A}$-fractions and $d$ in $B$ satisfies $C^{\circ} \neq C$ with

$$
C:=B / d B, \quad C^{\circ}:=B^{\circ} / d B
$$

then $C$ is a ring of $\mathcal{A}$-fractions with proper ideal $C^{\circ}$ and $C_{m, n}:=B_{m, n} / d B_{m, n}$.

(iii) If $B$ is a ring of $\mathcal{A}$-fractions and $c, d$ in $B$ satisfy $C^{\circ} \neq C$ with

$$
C=B\langle c / d\rangle:=B_{1,0} /\left(d \xi_{1}-c\right), \quad C^{\circ}:=\left(B^{\circ}\right) B\langle c / d\rangle
$$

then $C$ is a ring of $\mathcal{A}$-fractions with proper ideal $C^{\circ}$ and $C_{m, n}:=B_{m+1, n} /\left(d \xi_{1}-c\right)$.

(iv) If $B$ is a ring of $\mathcal{A}$-fractions and $c, d$ in $B$ satisfy $C^{\circ} \neq C$ with

$$
C=B[[c / d]]_{s}:=B_{0,1} /\left(d \rho_{1}-c\right), \quad C^{\circ}:=\left(B^{\circ}, \rho_{1}\right) B_{0,1} /\left(d \rho_{1}-c\right),
$$

and $\left(B^{\circ}, \rho_{1}\right)$ the ideal generated by $B^{\circ}$ and $\rho_{1}$, then $C$ is a ring of $\mathcal{A}$-fractions with proper ideal $C^{\circ}$ and $C_{m, n}:=B_{m, n+1} /\left(d \rho_{1}-c\right)$.

In all cases define $C_{m, n}^{\circ}$ as $\left(C^{\circ}, \rho\right) C_{m, n}$.

Definition 4.1.5 (Weierstrass system). Let $\mathcal{A}$ be a separated pre-Weierstrass system. Call $\mathcal{A}$ a separated Weierstrass system if it satisfies (c) below for any $\operatorname{ring} C$ of $\mathcal{A}$ fractions.

(c) If $f=\sum_{\mu, \nu} \bar{c}_{\mu \nu} \xi^{\mu} \rho^{\nu}$ is in $C_{m, n}$ with the $\bar{c}_{\mu \nu}$ in $C$, then there is a finite set $J \subset \mathbb{N}^{m+n}$ and for each $(\mu, v) \in J$ there is a $g_{\mu \nu} \in C_{m, n}^{\circ}$ such that

$$
f=\sum_{(\mu, \nu) \in J} \bar{c}_{\mu \nu} \xi^{\mu} \rho^{\nu}\left(1+g_{\mu \nu}\right) .
$$

Definition 4.1.6 (Analytic structure). Let $\mathcal{A}=\left\{A_{m, n}\right\}$ be a separated Weierstrass system, and let $K$ be a valued field. A separated analytic $\mathcal{A}$-structure on $K$ is a collection of homomorphisms $\left\{\sigma_{m, n}\right\}_{m, n \in \mathbb{N}}$ such that, for each $m, n \geq 0, \sigma_{m, n}$ is a homomorphism from $A_{m, n}$ to the ring of $K^{\circ}$-valued functions on $\left(K^{\circ}\right)^{m} \times\left(K^{\circ \circ}\right)^{n}$ and such that: 
(1) $I \subset \sigma_{0,0}^{-1}\left(K^{\circ \circ}\right)$,

(2) $\sigma_{m, n}\left(\xi_{i}\right)=$ the $i$-th coordinate function on $\left(K^{\circ}\right)^{m} \times\left(K^{\circ \circ}\right)^{n}, i=1, \ldots, m$, and $\sigma_{m, n}\left(\rho_{j}\right)=$ the $(m+j)$-th coordinate function on $\left(K^{\circ}\right)^{m} \times\left(K^{\circ \circ}\right)^{n}, j=1, \ldots, n$,

(3) $\sigma_{m, n+1}$ extends $\sigma_{m, n}$, where we identify in the obvious way functions on $\left(K^{\circ}\right)^{m} \times$ $\left(K^{\circ \circ}\right)^{n}$ with functions on $\left(K^{\circ}\right)^{m} \times\left(K^{\circ \circ}\right)^{n+1}$ that do not depend on the last coordinate, and $\sigma_{m+1, n}$ extends $\sigma_{m, n}$ similarly.

We have given the basic definitions of this section. A reader who wants to skip the proofs and the analysis of analytic structures can proceed directly with the examples in Section 4.4, with Section 4.5, and with the (model-theoretic) results of Section 6, having as well a look at Section 5. In Section 4.3 we give the basic definitions of strictly convergent analytic structures, which are simpler but less powerful.

Remark 4.1.7 (Units). If $\mathcal{A}=\left\{A_{m, n}\right\}_{m, n \in \mathbb{N}}$ is a separated pre-Weierstrass system and $f=1+g \in A_{m, n}$ with $g \in A_{m, n}^{\circ}$, then $f$ is regular of degree 0 and, by Weierstrass Division, $f$ is a unit in $A_{m, n}$. Moreover, $f \in A_{m, n}$ is a unit if and only if $f$ is of the form $c+g$ for some unit $c \in A$ and some $g \in A_{\sim}^{\circ} \underset{\sim}{\circ}$. Indeed, since $f$ is a unit there exists $h$ in $A_{m, n}$ such that $f h=1$, hence, $\tilde{f} \tilde{h}=1$ in $\widetilde{A}$, with $\sim$ as in Definition 4.1.2 (iv). Hence, $\tilde{f}$ is a unit in $\widetilde{A}_{m, n}$, hence $\widetilde{f}$ is a unit in $\widetilde{A}[x][[\rho]]$ and $\widetilde{f}$ is in $\widetilde{A}+\rho \widetilde{A}[x][[\rho]]$.

Remark 4.1.8 (Noetherianness). (i) If $I$ is a finitely generated ideal, then, in Definition 4.1.1, one has $B_{1}=(I, \rho) A[[\xi, \rho]]$ and $B_{2}=\left(I, \rho_{1}, \ldots, \rho_{n-1}, \rho_{n}^{d+1}\right) A[[\xi, \rho]]$.

(ii) While we did not require $A$ to be Noetherian, (see 4.4.7) for an example) it follows from (c) of Definition 4.1.5 that if $\mathcal{A}$ is a separated Weierstrass system and $f=\sum_{\mu, \nu} a_{\mu \nu} \xi^{\mu} \rho^{\nu} \in A_{m, n}$ then the ideal of $A$ generated by the $a_{\mu \nu}$ is finitely generated.

(iii) We refer to condition (c) of Definition 4.1.5 as a Strong Noetherian Property as it implies that all the coefficients of $f$ can be written as linear combinations of finitely many, and if the coefficient is "small", the corresponding coefficients of the linear combination are also small. Example 4.4(7) below shows that this does not require the ring $A$ to be Noetherian. Similarly, we refer to Theorem 4.2.15 as a Strong Noetherian Property, even though it does not imply that the rings $A_{m, n}$ are Noetherian.

As usual, Weierstrass preparation is a consequence of Weierstrass Division.

Remark 4.1.9 (Weierstrass Preparation). Let the family $\left\{A_{m, n}\right\}$ be a separated Weierstrass system. With the notation of Definition 4.1.3, we obtain the following for $f$ in $A_{m, n}$ :

(i) If $f$ is regular in $\xi_{m}$ of degree $d$, then there exist: a unique unit $u$ of $A_{m, n}$ and a unique monic polynomial $P \in A_{m-1, n}\left[\xi_{m}\right]$ of degree $d$ such that $f=u \cdot P$.

(ii) If $f$ is regular in $\rho_{n}$ of degree $d$, then there exist: a unique unit $u$ of $A_{m, n}$ and a unique monic polynomial $P \in A_{m, n-1}\left[\rho_{n}\right]$ of degree $d$ such that $f=u \cdot P$; in addition, $P$ is regular in $\rho_{n}$ of degree $d$.

This can be seen by dividing $\xi_{m}^{d}$ (respectively, $\rho_{n}^{d}$ ) by $f \in A_{m, n}$, as in [LR1, Corollary 2.3.3]. 
For $F$ a valued field and $f=\sum_{\mu, \nu} c_{\mu \nu} \xi^{\mu} \rho^{\nu}$ in $F[[\xi, \rho]]$, the Gauss norm of $f$ is written $\|f\|$ and defined as $\sup _{\mu \nu}\left|c_{\mu \nu}\right|$ if this supremum exists in $|F|$ and is not defined otherwise.

Remark 4.1.10 (Gauss norm). In the case that $A=F^{\circ}$ and $I=F^{\circ \circ}$ with $F$ a valued field, condition (c) of Definition 4.1.5 guarantees that the Gauss norm on $A_{m, n}$ is defined and moreover if $f \in A_{m, n}$ is nonzero then there is $c \in F$ such that $c f \in A_{m, n}$ and $\|c f\|=1$. Conversely, in this case $\left(A=F^{\circ}, I=F^{\circ}\right)$ if the $A_{m, n}$ satisfy Definitions 4.1.2 and 4.1.3 and for every $0 \neq f \in A_{m, n}$ there is an element $c \in F$ such that cf $\in A_{m, n}$ and $\|c f\|=1$, then condition (c) of Definition 4.1.5 follows from conditions (a), (b) and Definition 4.1.2 This fact (which we do not use) can be proved along the lines of the proof of Theorem 4.2.15

Remark 4.1.11. (i) As in the real case, it follows by Weierstrass Division that if $\xi_{i}$ (respectively $\rho_{j}$ ) divides $f \in A_{m, n}$ in $A[[\xi, \rho]]$, then $\xi_{i}$ (respectively $\rho_{j}$ ) divides $f$ in $A_{m, n}$.

(ii) As in the real case, if $f=\sum_{\mu \nu} \bar{f}_{\mu \nu}(\xi, \rho)\left(\xi^{\prime \prime}\right)^{\mu}\left(\rho^{\prime \prime}\right)^{\nu} \in A_{m^{\prime}, n^{\prime}}$ and

$$
I_{1}:=\left\{(\mu, v): \mu_{i} \geq d, v_{j} \geq d, \text { for all } i, j\right\},
$$

then

$$
f_{1}:=\sum_{(\mu, v) \in I_{1}} \bar{f}_{\mu \nu}(\xi, \rho)\left(\xi^{\prime \prime}\right)^{\mu}\left(\rho^{\prime \prime}\right)^{v}
$$

is an element of $A_{m^{\prime}, n^{\prime}}$. Similarly, for each $i, j$ and $\ell$,

$$
\sum_{\mu, \nu \text { with } \mu_{i}=\ell} \bar{f}_{\mu \nu}(\xi, \rho)\left(\xi^{\prime \prime}\right)^{\mu}\left(\rho^{\prime \prime}\right)^{v} \quad \text { and } \sum_{\mu, v \text { with } v_{j}=\ell} \bar{f}_{\mu v}(\xi, \rho)\left(\xi^{\prime \prime}\right)^{\mu}\left(\rho^{\prime \prime}\right)^{v}
$$

are elements of $A_{m^{\prime}, n^{\prime}}$. By (i) above, we can divide these series by $\xi_{i}^{\ell}$ (respectively, $\rho_{j}^{\ell}$ ).

(iii) Weierstrass Division guarantees that the rings $A_{m, n}$ are closed under Weierstrass changes of variables among the $\xi_{i}$ and among the $\rho_{j}$, but not in general under changes of variables that mix the $\xi_{i}$ and the $\rho_{j}$.

In Section 5.6 we will consider a variant of separated Weierstrass systems, by requiring some additional conditions. We will term these "strong separated Weierstrass systems".

Lemma 4.1.12. If $\mathcal{A}$ is a (strong) separated Weierstrass system, and $C$ is a ring of $\mathcal{A}$ fractions, then the family $\left\{C_{m, n}\right\}$ is a separated $\left(C, C^{\circ}\right)$-system which is a (strong) separated Weierstrass system (cf. Section 5.6 for "strong").

Proof. Since axiom (c) for $\left\{C_{m, n}\right\}$ follows at once from (c) for $\mathcal{A}$ and from (v) of Definition 4.1.2 only axioms (a) and (b) for the $C_{m, n}$ need proof, which we do by induction on the definition of $C$. Suppose that $C$ is $A\langle c / d\rangle$ and $f \in C_{m, n}$ is regular in $\xi_{m}$ of degree $s$. Then

$$
f \equiv \xi_{m}^{s}+F_{1} \xi_{m}^{s-1}+\cdots+F_{s} \bmod (I, \rho, c-\eta d)
$$


Let $F \in C_{m+1, n}$ satisfy $f \equiv F \bmod (c-\eta d)$. It may be that $F$ is not regular in $\xi_{m}$ of degree $s$. However, using the condition $\left(A_{m+1, n}\right)^{\sim} \subset \widetilde{A}[\xi, \eta][[\rho]]$ of Definition 4.1.2. we see that there is a finite $\operatorname{sum} \sum_{i=1}^{\ell}(c-\eta d) b_{i} \xi_{m}^{s+i}$ such that, taking $G=$ $F-\sum_{i=1}^{\ell}(c-\eta d) b_{i} \xi_{m}^{s+i}$, we have $f \equiv G \bmod (I, \rho, c-\eta d)$ and $G$ regular in $\xi_{m}$ of degree $s$. The other case is similar.

\subsection{The Strong Noetherian Property for separated Weierstrass systems}

Axiom (c) of Definition 4.1.5 is a kind of Noetherian property. In order to fully exploit it towards quantifier elimination, we have to work locally using the rings of fractions, defined in the previous subsection, and Laurent rings, defined below. The aim of this subsection is Theorem 4.2.15. which uses the full strength of the formalisms of Section 4.1 and of this section. (See also Remark 4.2.16.)

In this subsection, $\mathcal{A}$ is a separated Weierstrass system, as always over $(A, I)$.

First we elaborate some more on rings of $\mathcal{A}$-fractions.

Definition 4.2.1 (Defining formula). Let $C$ be a ring of $\mathcal{A}$-fractions. Call an expression a defining formula for $C$ when it can inductively be obtained by the following steps:

(i) The expression $(1=1)$ is a defining formula for $A$.

(ii) In case (ii) of Definition 4.1.4 if $\varphi_{B}$ is a defining formula for $B$, then

$$
\varphi_{B} \wedge(d=0)
$$

is a defining formula for $C$.

(iii) In case (iii) of Definition 4.1.4, if $\varphi_{B}$ is a defining formula for $B$, then

$$
\varphi_{B} \wedge(|c| \leq|d|) \wedge(d \neq 0)
$$

is a defining formula for $C$.

(iv) In case (iv) of Definition 4.1.4, if $\varphi_{B}$ is a defining formula for $B$, then

$$
\varphi_{B} \wedge(|c|<|d|) \wedge(c \neq 0)
$$

is a defining formula for $C$.

Definition 4.2.2 (System of rings of fractions). Let $\mathcal{A}$ be a separated $(A, I)$-system. If $C$ is a ring of $\mathcal{A}$-fractions and $c, d \in C$, then let $\mathcal{D}_{c, d}(C)$ be the set of rings of fractions among $C / d C, C\langle c / d\rangle, C[[d / c]]_{s}$ if this set is nonempty and let $\mathcal{D}_{c, d}(C)$ be $\{C\}$ otherwise. Define the concept of a system of rings of $\mathcal{A}$-fractions inductively as follows. $\mathcal{F}=\{A\}$ is a system of rings of $\mathcal{A}$-fractions. If $\mathcal{F}_{0}$ is a system of rings of $\mathcal{A}$-fractions and $C \in \mathcal{F}_{0}, c, d \in C$, then

$$
\mathcal{F}:=\left(\mathcal{F}_{0} \backslash\{C\}\right) \cup \mathcal{D}_{c, d}(C)
$$

is a system of rings of $\mathcal{A}$-fractions. 
Definition 4.2.3. Let $\mathcal{V}$ be the theory of the valuation rings of valued fields in the language of valued rings. For any commutative ring $C$ with unit and with fixed proper ideal $C^{\circ}$ (that is, $C^{\circ} \neq C$ ), define

$$
\mathcal{V}(C):=\mathcal{V} \cup\{|a| \leq 1: a \in C\} \cup\left\{|b|<1: b \in C^{\circ}\right\} .
$$

Lemma-Definition 4.2.4. With the notation from Definition 4.2 .3 and with a system $\mathcal{F}$ of rings of $\mathcal{A}$-fractions, it follows that

$$
\mathcal{V}(A) \vdash \bigvee_{C \in \mathcal{F}} \varphi_{C} \quad \text { and } \quad \mathcal{V}(A) \vdash \bigwedge_{C \neq C^{\prime} \in \mathcal{F}} \neg\left(\varphi_{C} \wedge \varphi_{C^{\prime}}\right) \text {. }
$$

If moreover $\mathcal{A}$ is a separated Weierstrass system and $\sigma$ is a separated analytic $\mathcal{A}$-structure on $K$, then there is exactly one $C \in \mathcal{F}$ such that $K^{\circ} \models \varphi_{C}$ under the interpretation provided by $\sigma$. We call such $\varphi_{C}$ compatible with $\sigma$. We call a ring of $\mathcal{A}$-fractions $C$ compatible with $\sigma$ when it has a defining formula that is compatible with $\sigma$.

Proof. This holds by the definitions.

Next we generalize the notion of rings of $\mathcal{A}$-fractions to that of Laurent rings. This notion will mainly be used for Theorem 4.2.15

Definition 4.2.5 (Laurent rings). Let $C$ be a ring of $\mathcal{A}$-fractions. Inductively define the concept that a $C_{m, n}$-algebra $C^{\prime}$ is a Laurent ring over $C_{m, n}$ with proper ideal $C^{\prime \circ}$ as follows.

(i) $C_{m, n}$ is a Laurent ring over $C_{m, n}$ with proper ideal $C_{m, n}^{\circ}$ (cf. Definition 4.1.4).

(ii) Let $J$ be an ideal of $C_{m+M, n+N}$. If $B=C_{m+M, n+N} / J$ is a Laurent ring over $C_{m, n}$, if $f \in C_{m+M, n+N}$, and if $C^{\prime \circ} \neq C^{\prime}$, where

$$
\begin{aligned}
C^{\prime} & =B\langle 1 / f\rangle:=C_{m+M+1, n+N} /\left(J, \xi_{m+M+1} f-1\right), \\
C^{\prime \circ} & :=\left(C^{\circ}+\left(\rho_{1}, \ldots, \rho_{n+N}\right)\right) C_{m+M+1, n+N} /\left(J, \xi_{m+M+1} f-1\right),
\end{aligned}
$$

then $C^{\prime}$ is a Laurent ring over $C_{m, n}$ with proper ideal $C^{\prime \circ}$.

(iii) Let $J$ be an ideal of $C_{m+M, n+N}$. If $B=C_{m+M, n+N} / J$ is a Laurent ring over $C_{m, n}$, if $f \in C_{m+M, n+N}$, and if $C^{\prime \circ} \neq C^{\prime}$, where

$$
\begin{aligned}
C^{\prime} & =B[[f]]_{s}:=C_{m+M, n+N+1} /\left(J, \rho_{n+N+1}-f\right), \\
C^{\prime \circ} & :=\left(C^{\circ}+\left(\rho_{1}, \ldots, \rho_{n+N+1}\right)\right) C_{m+M, n+N+1} /\left(J, \rho_{n+N+1}-f\right),
\end{aligned}
$$

then $C^{\prime}$ is a Laurent ring over $C_{m, n}$ with proper ideal $C^{\prime \circ}$.

Definition 4.2.6. Let $C$ be a ring of $\mathcal{A}$-fractions. For any Laurent ring $C^{\prime}$ over $C_{m, n}$ there exists an ideal $J$ of some $C_{M+m, N+n}$ such that $C^{\prime}=C_{M+m, N+n} / J$. Define then

$$
\begin{aligned}
C_{m_{1}, n_{1}}^{\prime} & :=C_{M+m+m_{1}, N+n+n_{1}} / J C_{M+m+m_{1}, N+n+n_{1}}, \\
\left(C_{m_{1}, n_{1}}^{\prime}\right)^{\circ} & :=\left(C^{\circ},\left(\rho_{1}, \ldots, \rho_{N+n+n_{1}}\right)\right) C_{m_{1}, n_{1}}^{\prime} .
\end{aligned}
$$


The following is the analogue for Laurent rings of Lemma 4.1.12. We will not need it. The proof is similar to that of Lemma 4.1.12

Lemma 4.2.7. If $\mathcal{A}$ is a (strong) separated Weierstrass system, and $B$ is a Laurent ring over $C_{m, n}$ for some ring of $\mathcal{A}$-fractions $C$, then the family $\left\{B_{m, n}\right\}$ is a (strong) separated Weierstrass system over $\left(B, B^{\circ}\right)$ (cf. Section 5.6 for the definition of "strong").

Definition 4.2.8 (Defining formula). Let $C$ be a ring of $\mathcal{A}$-fractions. Call an expression a defining formula for $B$, with $B$ a Laurent ring over $C_{m, n}$, when it can inductively be obtained by the following steps:

(i) The expression $(1=1)$ is a defining formula for $C_{m, n}$.

(ii) In case (ii) of Definition 4.2.5 if $\varphi_{C^{\prime}}$ is a defining formula of $C^{\prime}$, then

$$
\varphi_{C^{\prime}} \wedge(|f| \geq 1)
$$

is a defining formula for $B:=C^{\prime}\langle 1 / f\rangle$.

(iii) In case (iii) of Definition 4.2.5, if $\varphi_{C^{\prime}}$ is a defining formula of $C^{\prime}$, then

$$
\varphi_{C^{\prime}} \wedge(|f|<1)
$$

is a defining formula for $B:=C^{\prime}[[f]]_{s}$.

Definition 4.2.9 (Covering family). Let $C$ be a ring of $\mathcal{A}$-fractions. Note that the theory $\mathcal{V}(C)$ is well defined by Definition 4.2.3 and Lemma 4.1.12 We call a finite family $\mathcal{F}$ of Laurent rings over $C_{m, n}$ a covering family if

$$
\mathcal{V}(C) \vdash \bigvee_{B \in \mathcal{F}} \varphi_{B}
$$

The family is a disjointly covering family if in addition $\neg\left(\varphi_{B} \wedge \varphi_{B^{\prime}}\right)$ is a theorem of $\mathcal{V}(C)$ for all $B \neq B^{\prime} \in \mathcal{F}$.

By construction, we have the following:

Lemma 4.2.10. Let $\sigma$ be a separated analytic $\mathcal{A}$-structure on $K$ and let $C$ be a ring of $\mathcal{A}$-fractions compatible with $\sigma$. Any defining formula $\varphi_{B}$ of any Laurent ring $B$ over $C_{m, n}$ defines in a natural way a subset $X_{\varphi_{B}}$ of $\left(K^{\circ}\right)^{m} \times\left(K^{\circ \circ}\right)^{n}$, which may be empty. Moreover, for any covering family $\mathcal{F}$ of Laurent rings $B$ over $C_{m, n}$ with formulas $\varphi_{B}$, the union of the sets $X_{\varphi_{B}}$ equals $\left(K^{\circ}\right)^{m} \times\left(K^{\circ \circ}\right)^{n}$.

Definition 4.2.11 (Units). Let $C$ be a Laurent ring over $A_{m, n}$. (Any Laurent ring over a ring of $\mathcal{A}$-fractions is a Laurent ring over some $A_{m, n}$.) We call $f \in C$ a $C$-unit if

$$
\mathcal{V}(C), \varphi_{C} \vdash|f| \geq 1 \text {. }
$$

We recall the following definition from [LL2, Section 3.12], which is used in several proofs. 
Definition 4.2.12 (Preregular). Let $C$ be a Laurent ring over $A_{m, n}$ and, using the notation of Definition 4.1.2. let $f=\sum_{\mu \nu} c_{\mu \nu}\left(\xi^{\prime \prime}\right)^{\mu}\left(\rho^{\prime \prime}\right)^{\nu} \in C_{m^{\prime}-m, n^{\prime}-n}$ where the $c_{\mu \nu}$ are in $C$. We call $f$ preregular in $\left(\xi^{\prime \prime}, \rho^{\prime \prime}\right)$ of degree $\left(\mu_{0}, \nu_{0}\right)$ if $c_{\mu_{0} \nu_{0}}=1$ and $c_{\mu \nu} \in$ $\left(C_{m^{\prime}-m, n^{\prime}-n}\right)^{\circ}$ for all $v$ lexicographically $<\nu_{0}$ and for all $\left(\mu, \nu_{0}\right)$ with $\mu$ lexicographically $>\mu_{0}$.

Remark 4.2.13. If $f$ is preregular of degree $\left(\mu_{0}, 0\right)$ then a Weierstrass change of variables among the $\xi_{i}, i=m+1, \ldots, m^{\prime}$ will make $f$ regular in $\xi_{m^{\prime}}$. Similarly, if $f$ is preregular of degree $\left(0, \nu_{0}\right)$ then a Weierstrass change of variables among the $\rho_{j}$, $j=n+1, \ldots, n^{\prime}$ will make $f$ regular in $\rho_{n^{\prime}}$.

The following lemma is needed in the proof of Theorem 4.2.15. The proofs of this lemma and Theorem 4.2.15 are similar to the proofs of Lemma 3.2.1 and Theorem 3.2.2. with some additional complications.

Lemma 4.2.14. Let $\left\{A_{m, n}\right\}$ be a separated Weierstrass system and, using the notation of Definition 4.1.2 let

$$
f=\sum_{\mu, \nu} \bar{f}_{\mu \nu}(\xi, \rho)\left(\xi^{\prime \prime}\right)^{\mu}\left(\rho^{\prime \prime}\right)^{\nu} \in A_{m^{\prime}, n^{\prime}}
$$

where the $\bar{f}_{\mu \nu}(\xi, \rho)$ are in $A_{m, n}$. There is a system $\mathcal{F}$ of rings of $\mathcal{A}$-fractions, and for each $A^{\prime} \in \mathcal{F}$ there is a finite, disjointly covering family of Laurent rings $C$ over $A_{m, n}^{\prime}$, such that for each $C$ there is a finite set $J_{C}$, and $C$-units $u_{C \mu \nu}$ and functions $g_{C \mu \nu} \in C_{m^{\prime}-m, n^{\prime}-n}$ for $(\mu, \nu) \in J_{C}$, such that

$$
f=\sum_{(\mu, \nu) \in J_{C}} \bar{f}_{\mu \nu} u_{C \mu \nu} g_{C \mu \nu}
$$

as an element of $C_{m^{\prime}-m, n^{\prime}-n}$.

In the case that $A$ is a valuation ring with maximal ideal $A^{\circ}$ we can take $\mathcal{F}=\{A\}$. In the case that $m=0$ or $n=0$ we can take the family of Laurent rings corresponding to $A^{\prime} \in \mathcal{F}$ to be just $\left\{A_{m, n}^{\prime}\right\}$.

Proof. Let $f$ be as above, say (using the notation of Definition 4.1.2

$$
f=\sum_{\mu^{\prime}, v^{\prime}} \bar{a}_{\mu^{\prime} v^{\prime}}\left(\xi^{\prime}\right)^{\mu^{\prime}}\left(\rho^{\prime}\right)^{v^{\prime}}
$$

with the $\bar{a}_{\mu^{\prime} v^{\prime}}$ in $A$. Then by condition (c) (Definition 4.1 .5 we have

$$
f=\sum_{\left(\mu^{\prime}, v^{\prime}\right) \in J} \bar{a}_{\mu^{\prime} v^{\prime}}\left(\xi^{\prime}\right)^{\mu^{\prime}}\left(\rho^{\prime}\right)^{v^{\prime}}\left(1+g_{\mu^{\prime}, v^{\prime}}\right)
$$

with the $g_{\mu^{\prime}, v^{\prime}}$ in $A_{m^{\prime}, n^{\prime}}^{\circ}$ for some finite $J \subset \mathbb{N}^{m^{\prime}+n^{\prime}}$. Hence, splitting into finitely many cases corresponding to a system $\mathcal{F}$ of rings of $\mathcal{A}$-fractions, and considering each $A^{\prime} \in \mathcal{F}$ separately, we may assume that $\bar{a}_{\mu_{0}^{\prime} v_{0}^{\prime}}=1$ and that $f$ is preregular in $\left(\xi^{\prime}, \rho^{\prime}\right)$ of degree $\left(\mu_{0}^{\prime}, v_{0}^{\prime}\right)$. (In the case that $A$ is a valuation ring, there is no need to split up into cases to 
find, and "factor out", the "dominant" coefficient $\bar{a}_{\mu_{0}^{\prime} \nu_{0}^{\prime}}$. Let $\mu_{0}^{\prime}=\left(\mu_{0}^{\prime \prime}, \mu_{0}\right)$ and $v_{0}^{\prime}=$ $\left(v_{0}^{\prime \prime}, v_{0}\right)$ and write

$$
f=\sum_{(\mu, v)} \bar{f}_{\mu v}(\xi, \rho)\left(\xi^{\prime \prime}\right)^{\mu}\left(\rho^{\prime \prime}\right)^{v}
$$

with the $\bar{f}_{\mu, \nu}$ in $A_{m, n}$. Then $\bar{f}_{\mu_{0} \nu_{0}}$ is preregular in $(\xi, \rho)$ of degree $\left(\mu_{0}^{\prime \prime}, v_{0}^{\prime \prime}\right)$ and, writing

$$
\bar{f}_{\mu_{0} \nu_{0}}=\sum_{v^{\prime \prime}} \bar{f}_{\mu_{0} \nu_{0} v^{\prime \prime}}(\xi)(\rho)^{v^{\prime \prime}}
$$

we see that $\bar{f}_{\mu_{0} v_{0} v_{0}^{\prime \prime}}(\xi)$ is preregular in $\xi$ of degree $\mu_{0}^{\prime \prime}$.

If $m=0$ or $n=0$, a Weierstrass change of variables among the $\rho_{j}$ (respectively the $\xi_{i}$ ) will make $\bar{f}_{\mu_{0} v_{0}}$ regular in $\rho_{n}$ (respectively $\xi_{m}$ ) and no "splitting up" into Laurent rings is needed before doing Weierstrass division by $\bar{f}_{\mu_{0} \nu_{0}}$ (below). In the general case, consider the two Laurent rings $C_{1}$ and $C_{2}$ defined by the conditions $\left|\bar{f}_{\mu_{0} v_{0} v^{\prime \prime}}\right|<1$ and $\left|\bar{f}_{\mu_{0} \nu_{0} \nu^{\prime \prime}}\right| \geq 1$, respectively.

On $C_{1}$, using $\lambda$ to denote the new variable (of the second kind, i.e. a " $\rho$ " variable) $\bar{f}_{\mu_{0} v_{0} v^{\prime \prime}}-\lambda=0$. After a Weierstrass change of variables among $\xi_{1}, \ldots, \xi_{m}$, we may assume that $\bar{f}_{\mu_{0} v_{0} v^{\prime \prime}}-\lambda$ is regular in $\xi_{m}$ of degree $s$, say. Then in $\left(C_{1}\right)_{m^{\prime}-m, n^{\prime}-n}$ we have

$$
f=R_{0}\left(\hat{\xi}, \xi^{\prime \prime}, \rho, \rho^{\prime \prime}, \lambda\right)+\xi_{m} R_{1}\left(\hat{\xi}, \xi^{\prime \prime}, \rho, \rho^{\prime \prime}, \lambda\right)+\cdots+\xi_{m}^{s-1} R_{s-1}\left(\hat{\xi}, \xi^{\prime \prime}, \rho, \rho^{\prime \prime}, \lambda\right),
$$

where $\hat{\xi}:=\left(\xi_{1}, \ldots, \xi_{m-1}\right)$. We now complete the proof in this case by induction on $s$ and induction on $(m, n)$, ordered lexicographically, as in the proof of Lemma 3.2.1 $((m, n)$ has been reduced to $(m-1, n+1)$.)

On $C_{2}$, using $\eta$ for the new variable, $\eta \bar{f}_{\mu_{0} v_{0} v^{\prime \prime}}-1=0$. After a Weierstrass change of variables among $\xi_{1}, \ldots, \xi_{m}, \eta$, we may assume that $\eta \bar{f}_{\mu_{0} \nu_{0} v^{\prime \prime}}-1$ is regular in $\eta$ of degree $s_{1}$, say. Considering $\eta f$ and $\eta f_{\mu_{0} \nu_{0}}$, after replacing the coefficient $\eta \bar{f}_{\mu_{0} v_{0} v_{0}^{\prime \prime}}$ of $\rho^{\nu_{0}^{\prime \prime}}$ by $1, \eta \bar{f}_{\mu_{0} \nu_{0}}$ is preregular in $\rho$ of degree $v_{0}^{\prime \prime}$. After a Weierstrass change of variables among $\rho_{1}, \ldots, \rho_{m}$, we may assume that $\eta \bar{f}_{\mu_{0} \nu_{0}}$ is regular in $\rho_{n}$, of degree $s_{2}$, say. Doing Weierstrass division twice, once by $\eta \bar{f}_{\mu_{0} \nu_{0} \nu^{\prime \prime}}-1$ and once by $\eta \bar{f}_{\mu_{0} \nu_{0}}$, we see that in $\left(C_{2}\right)_{m^{\prime}-m, n^{\prime}-n}$ we have

$$
\eta f=Q \cdot \eta \bar{f}_{\mu_{0} v_{0}}+\sum_{i<s_{1}, j<s_{2}} R_{i j}\left(\xi, \xi^{\prime \prime}, \hat{\rho}, \rho^{\prime \prime}\right) \eta^{i} \rho_{n}^{j},
$$

where $\hat{\rho}=\left(\rho_{1}, \ldots, \rho_{n-1}\right)$. One again completes the proof by induction on $s_{1} s_{2}$ and on $(m, n)$, ordered lexicographically, as in the proof of Lemma 3.2.1 $((m, n)$ has been reduced to $(m, n-1)$.)

We continue to use the notation of Definition 4.1 .2 
Theorem 4.2.15 (The Strong Noetherian Property for separated Weierstrass systems). Let $\left\{A_{m, n}\right\}$ be a separated Weierstrass system and let

$$
f=\sum_{\mu, \nu} \bar{f}_{\mu \nu}(\xi, \rho)\left(\xi^{\prime \prime}\right)^{\mu}\left(\rho^{\prime \prime}\right)^{v} \in A_{m^{\prime}, n^{\prime}}
$$

with the $\bar{f}_{\mu \nu}(\xi, \rho)$ in $A_{m, n}$. There is a finite system $\mathcal{F}$ of rings of A-fractions, and for each $A^{\prime} \in \mathcal{F}$ there is a finite, disjointly covering family of Laurent rings $C$ over $A_{m, n}^{\prime}$ such that for each $C$ there is a finite set $J_{C}$ and $C$-units $u_{C \mu \nu} \in C$ and functions $h_{C \mu \nu} \in$ $C_{m^{\prime}-m, n^{\prime}-n}^{\circ}$ for $(\mu, v) \in J_{C}$ such that

$$
f=\sum_{(\mu, v) \in J_{C}} \bar{f}_{\mu \nu}(\xi, \rho)\left(\xi^{\prime \prime}\right)^{\mu}\left(\rho^{\prime \prime}\right)^{\nu} u_{C \mu \nu}\left(1+h_{C \mu \nu}\right)
$$

as an element of $C_{m^{\prime}-m, n^{\prime}-n}$.

When $A$ is a valuation ring we can take the system $\mathcal{F}=\{A\}$. If $m=0$ or $n=0$ we can take the family of Laurent rings corresponding to $A^{\prime} \in \mathcal{F}$ to be just $\left\{A_{m, n}^{\prime}\right\}$. Hence, in the case that $A$ is a valuation ring and $m n=0$ no (nontrivial) rings of A-fractions or Laurent rings are needed.

Proof. By Lemma 4.2.14 we may assume we have written

$$
f=\sum_{(\mu, v) \in J_{C}} \bar{f}_{\mu \nu} u_{C \mu \nu} h_{C \mu \nu}
$$

with the $h_{C \mu \nu}$ in $C_{m^{\prime}, n^{\prime}}$. As in the proof of Theorem 3.2.2 choosing $d$ large enough, writing $k$ for $m^{\prime}-m+n^{\prime}-n$, and taking

$$
I_{1}:=\{1, \ldots, d\}^{k} \cup\left\{(\mu, v): \mu_{i} \geq d, v_{j} \geq d \text { for all } i, j\right\},
$$

we have

$$
f_{I_{1}}:=\sum_{(\mu, \nu) \in I_{1}} \bar{f}_{\mu \nu}(\xi, \rho)\left(\xi^{\prime \prime}\right)^{\mu}\left(\rho^{\prime \prime}\right)^{\nu}=\sum_{(\mu, \nu) \in\{1, \ldots, d\}^{k}} \bar{f}_{\mu \nu}\left(\xi^{\prime \prime}\right)^{\mu}\left(\rho^{\prime \prime}\right)^{\nu} u_{C \mu \nu}\left(1+g_{C \mu \nu}\right)
$$

with the $g_{C \mu \nu}$ in $\left(\xi^{\prime \prime}, C_{m^{\prime}, n^{\prime}}^{\circ}\right) C_{m^{\prime}, n^{\prime}}$. Since each $g_{C \mu \nu} \bmod \left(C_{m^{\prime}, n^{\prime}}\right)^{\circ}$ is a polynomial in $\xi^{\prime \prime}$ (cf. Definition 4.1.2), further increasing $d$, we may assume that each $g_{C \mu \nu}$ is in $\left(C_{m^{\prime}, n^{\prime}}\right)^{\circ}$. The proof is now completed exactly as that of Theorem 3.2.2 by induction on $m^{\prime}-m+$ $n^{\prime}-n$.

Remark 4.2.16. In many examples, for example when $A$ is Noetherian and complete in its $I$-adic topology and $A_{m, n}:=A\langle\xi\rangle[[\rho]]$ (cf. [CLR1], Section 2), or $A_{m, n}=S_{m, n}^{\circ}(E, K)$ (cf. [LR1]) we do not have to break up into pieces using rings of $\mathcal{A}$-fractions and Laurent rings, and the following stronger statement is true (using the notation of Definition 4.1.2):

- Let $f \in A_{m^{\prime}, n^{\prime}}$ and write $f=\sum_{\mu, \nu} \bar{f}_{\mu \nu}(\xi, \rho)\left(\xi^{\prime \prime}\right)^{\mu}\left(\rho^{\prime \prime}\right)^{v}$ with the $\bar{f}_{\mu \nu}$ in $A_{m, n}$. There is a finite set $J \subset \mathbb{N}^{m^{\prime}-m+n^{\prime}-n}$ and units of the form $1+g_{\mu \nu}$ with $g_{\mu \nu} \in A_{m^{\prime}, n^{\prime}}^{\circ}$ such that

$$
f=\sum_{(\mu, \nu) \in J} \bar{f}_{\mu \nu}(\xi, \rho)\left(\xi^{\prime \prime}\right)^{\mu}\left(\rho^{\prime \prime}\right)^{\nu}\left(1+g_{\mu \nu}\right)
$$


The Strong Noetherian Properties of Definition 4.1.5 and Theorem 4.2.15 follow immediately from this property. Our treatment would be less general but also simpler were we to take this condition as an axiom replacing the weaker axiom (c) of Definition 4.1.5. We would then not have to prove Theorem 4.2.15.

\subsection{Strictly convergent analytic structures}

We consider polynomial rings and power series rings in one kind of variables, written $\xi_{i}$, usually variants of Tate rings, hence the terminology "strictly convergent". In the separated case of the previous two subsections the $\rho$ variables (varying over $K^{\circ \circ}$ ) were used to witness strict inequalities. Furthermore, the second Weierstrass division axiom (Definition 4.1.3 (b)) enforced some additional completeness on the ring $A$, on rings of $\mathcal{A}$-fractions, and on fields $K$ with analytic $\mathcal{A}$-structure. In the strictly convergent case we will allow two possibilities which we distinguish by use of a designated element $\pi$ of $A$ : (i) $\pi \neq 1$ and in the interpretations given by fields $K$ with analytic $\mathcal{A}$-structure, $\pi$ is interpreted as a prime element of $K^{\circ}$, i.e. $\sigma(\pi)$ is an element of smallest positive order (see Definition 4.3.6(i)), and (ii) that the strictly convergent analytic structure is the strictly convergent part of a separated analytic structure and $\pi=1$. Except for this complication we follow the development of the previous two sections fairly closely.

Case (ii) is treated in Definition 4.3.6.ii). We first focus on case (i). Let $A$ and $I$ be as at the beginning of Section 4 . Let $\pi$ be a fixed element of $A$.

Definition 4.3.1 (System). A system $\mathcal{A}=\left\{A_{m}\right\}_{m \in \mathbb{N}}$ of $A$-algebras $A_{m}$, satisfying, for all $m \leq m^{\prime}$ :

(i) $A_{0}=A$,

(ii) $A_{m} \subset A\left[\left[\xi_{1}, \ldots, \xi_{m}\right]\right]$,

(iii) $A_{m}\left[\xi_{m+1}, \ldots, \xi_{m^{\prime}}\right] \subset A_{m^{\prime}}$

(iv) the image $\left(A_{m}\right) \stackrel{\sim}{\sim}$ of $A_{m}$ under the residue map $\sim: A\left[\left[\xi_{1}, \ldots, \xi_{m}\right]\right] \rightarrow \widetilde{A}\left[\left[\xi_{1}, \ldots, \xi_{m}\right]\right]$ is $\widetilde{A}[\xi]$,

(v) if $f \in A_{m^{\prime}}$, say $f=\sum_{\mu} \bar{f}_{\mu}(\xi)\left(\xi^{\prime \prime}\right)^{\mu}$, then the $\bar{f}_{\mu}$ are in $A_{m}$,

is called a strictly convergent $(A, I)$-system.

Definition 4.3.2 (Regular). Let $\mathcal{A}=\left\{A_{m}\right\}_{m \in \mathbb{N}}$ be a strictly convergent ( $A, I$ )-system. A power series $f$ in $A_{m}$ is called regular in $\xi_{m}$ of degree $d$ when $f$ is congruent in $A[[\xi]]$, modulo the ideal $\left\{\sum_{\mu} a_{\mu} \xi^{\mu}: a_{\mu} \in I\right\}$, to a monic polynomial in $\xi_{m}$ of degree $d$.

Definition 4.3.3 (Pre-Weierstrass system). Let $\mathcal{A}=\left\{A_{m}\right\}_{m \in \mathbb{N}}$ be a strictly convergent ( $A, I)$-system. Then $\mathcal{A}$ is called a strictly convergent pre-Weierstrass system when the usual Weierstrass Division Theorem holds in the $A_{m}$, namely, for $f, g \in A_{m}$ :

(a) if $f$ is regular in $\xi_{m}$ of degree $d$, then there exist uniquely determined elements $q \in A_{m}$ and $r \in A_{m-1}\left[\xi_{m}\right]$ of degree at most $d-1$ such that $g=q f+r$.

In fact, as in the separated case, we need to be able to work locally, using rings of fractions: 
Definition 4.3.4 (Rings of fractions). Let $\mathcal{A}=\left\{A_{m}\right\}_{m \in \mathbb{N}}$ be a strictly convergent $(A, I)$ system. Inductively define the concept that $C$ is a ring of $\mathcal{A}$-fractions with ideal $C^{\circ}$ and rings of strictly convergent functions $C_{m}, m \geq 0$, as follows:

(i) The ring $A$ is a ring of $\mathcal{A}$-fractions with ideal $I=A^{\circ}$ and rings of strictly convergent functions the $A_{m}$.

(ii) If $B$ is a ring of $\mathcal{A}$-fractions and $d \in B$ satisfies $C^{\circ} \neq C$ with

$$
C:=B / d B, \quad C^{\circ}:=B^{\circ} \cdot C,
$$

then $C$ is a ring of $\mathcal{A}$-fractions with ideal $C^{\circ}$ and strictly convergent functions $C_{m}=$ $B_{m} / d B_{m}$.

(iii) If $B$ is a ring of $\mathcal{A}$-fractions and $c, d \in B$ satisfy $C^{\circ} \neq C$ with

$$
C:=B\langle c / d\rangle:=B_{1} /\left(d \xi_{1}-c\right), \quad C^{\circ}:=B^{\circ} \cdot C,
$$

then $C$ is a ring of $\mathcal{A}$-fractions with ideal $C^{\circ}$ and strictly convergent functions $C_{m}=$ $B_{m+1} /\left(d \xi_{1}-c\right)$.

(iv) If $B$ is a ring of $\mathcal{A}$-fractions and $c, d \in B$ satisfy $C^{\circ} \neq C$ with

$$
C:=B\left\langle\frac{c}{\pi \cdot d}\right\rangle:=B_{1} /\left(d \cdot \pi \cdot \xi_{1}-c\right), \quad C^{\circ}:=B^{\circ} \cdot C,
$$

then $C$ is a ring of $\mathcal{A}$-fractions with ideal $C^{\circ}$ and strictly convergent functions $C_{m}=$ $B_{m+1} /\left(\pi \cdot d \cdot \xi_{1}-c\right)$.

Note that part (iv) above differs from the definition we made in the separated case; the notation $B\langle c / \pi \cdot d\rangle$ for the ring is reminiscent of the inequality $c<d$ which in our case (namely $\pi \neq 1$ ) is equivalent to $c \leq \pi \cdot d$.

Definition 4.3.5 (Weierstrass system). Let $\mathcal{A}$ be a strictly convergent pre-Weierstrass system. Call $\mathcal{A}$ a strictly convergent Weierstrass system if it satisfies (c) below for every ring $C$ of $\mathcal{A}$-fractions.

(c) If $f=\sum_{\mu} \bar{c}_{\mu} \xi^{\mu}$ is in $C_{m}$ with the $\bar{c}_{\mu}$ in $C$, then there is a finite set $J \subset \mathbb{N}^{m}$ and for $\mu \in J$ there is $g_{\mu} \in C_{m}^{\circ}$ such that

$$
f=\sum_{\mu \in J} \bar{c}_{\mu} \xi^{\mu}\left(1+g_{\mu}\right) .
$$

Definition 4.3.6 (Analytic structure).

(i) $(\pi \neq 1)$ Let $\mathcal{A}=\left\{A_{m}\right\}$ be a strictly convergent Weierstrass system, and let $K$ be a valued field. A strictly convergent analytic $\mathcal{A}$-structure on $K$ is a collection of homomorphisms $\left\{\sigma_{m}\right\}_{m \in \mathbb{N}}$ such that, for each $m \geq 0, \sigma_{m}$ is a homomorphism from $A_{m}$ to the ring of $K^{\circ}$-valued functions on $\left(K^{\circ}\right)^{m}$ satisfying:

(1) $I \subset \sigma_{0}^{-1}\left(K^{\circ \circ}\right)$ and $\sigma_{0}(\pi)$ is a prime element of $K^{\circ}$,

(2) $\sigma_{m}\left(\xi_{i}\right)=$ the $i$-th coordinate function on $\left(K^{\circ}\right)^{m}, i=1, \ldots, m$, 
(3) $\sigma_{m+1}$ extends $\sigma_{m}$ where we identify in the obvious way functions on $\left(K^{\circ}\right)^{m}$ with functions on $\left(K^{\circ}\right)^{m+1}$ that do not depend on the last coordinate.

(ii) $(\pi=1)$ Let $\mathcal{A}=\left\{A_{m, n}\right\}$ be a separated Weierstrass system and $K$ a field with separated analytic $\mathcal{A}$-structure $\sigma=\left\{\sigma_{m, n}\right\}$. We will define the strictly convergent analytic structure $\bar{\sigma}$ on $K$ associated to $\sigma$. Note that $\sigma$ induces morphisms $\sigma_{C_{m, 0}}$ from $C_{m, 0}$ to the ring of $K^{\circ}$-valued functions on $\left(K^{\circ}\right)^{m}$, for $C$ any ring of $\mathcal{A}$-fractions which is compatible with $\sigma$ (cf. Definitions 4.1.4, 4.2.4). Then $\bar{\sigma}$ is defined as the collection of homomorphisms $\sigma_{C_{m, 0}}$ for all $m \geq 0$ and all rings $C$ of $\mathcal{A}$-fractions which are compatible with $\sigma$.

In (ii) of Definition 4.3.6, the analytic structure does not come from a strictly convergent $(A, I)$-system, but from a richer system of the rings $C_{m, 0}$ for many $C$. However, for the separated analytic structure $\sigma^{\prime}$ with $\sigma^{\prime}$ as in Definition 4.5.6, $\bar{\sigma}^{\prime}$ contains no more data than the collection of the maps $\sigma_{m, 0}^{\prime}$. With a slight abuse of terminology we will also denote in this case the collection of the maps $\sigma_{m, 0}^{\prime}$ by $\bar{\sigma}^{\prime}$ and call $\bar{\sigma}^{\prime}$ the strictly convergent analytic structure on $K$ associated to $\sigma^{\prime}$.

In this paper we focus on case (i) $(\pi \neq 1)$ of Definition 4.3.6, studying case (ii) is much more subtle and will require new techniques.

Definition 4.3.7 (Defining formula). Let $C$ be a ring of $\mathcal{A}$-fractions (Definition 4.3.4). Call an expression a defining formula for $C$ when it can inductively be obtained by the following steps:

(i) The expression $(1=1)$ is a defining formula for $A$.

(ii) In case (ii) of Definition 4.3.4 if $\varphi_{B}$ is a defining formula for $B$, then

$$
\varphi_{B} \wedge(d=0)
$$

is a defining formula for $C$.

(iii) In case (iii) of Definition 4.3.4, if $\varphi_{B}$ is a defining formula for $B$, then

$$
\varphi_{B} \wedge(|c| \leq|d|) \wedge(d \neq 0)
$$

is a defining formula for $C$.

(iv) In case (iv) of Definition 4.3.4, if $\varphi_{B}$ is a defining formula for $B$, then

$$
\varphi_{B} \wedge(|c| \leq|\pi \cdot d|) \wedge(c \neq 0)
$$

is a defining formula for $C$.

Definition 4.3.8 (System of rings of fractions). Let $\mathcal{A}=\left\{A_{m}\right\}_{m \in \mathbb{N}}$ be a strictly convergent $(A, I)$-system. If $C$ is a ring of $\mathcal{A}$-fractions and $c, d \in C$, then let $\mathcal{D}_{c, d}(C)$ be the set of rings of fractions among $C / d C, C\langle c / d\rangle, C\langle d / \pi \cdot c\rangle$ if this set is nonempty and let $\mathcal{D}_{c, d}(C)$ be $\{C\}$ otherwise. Define the concept of a system of rings of $\mathcal{A}$-fractions inductively as follows. $\mathcal{F}=\{A\}$ is a system of rings of $\mathcal{A}$-fractions. If $\mathcal{F}_{0}$ is a system of rings of $\mathcal{A}$-fractions, and $C \in \mathcal{F}_{0}, 0 \neq c, 0 \neq d \in C$, then

$$
\mathcal{F}:=\left(\mathcal{F}_{0} \backslash\{C\}\right) \cup \mathcal{D}_{c, d}(C)
$$

is a system of rings of $\mathcal{A}$-fractions. 
Remark 4.3.9. Let $\mathcal{V}^{\prime}$ be the theory of the valuation rings of valued fields with prime element $\pi$, and let $\mathcal{A}$ be a strictly convergent $(A, I)$-system and $\mathcal{F}$ a system of rings of $\mathcal{A}$-fractions. Let

$$
\mathcal{V}^{\prime}(A):=\mathcal{V}^{\prime} \cup\{|a| \leq 1: a \in A\} \cup\left\{|b| \leq|\pi|: b \in A^{\circ}\right\} .
$$

Then

$$
\mathcal{V}^{\prime}(A) \vdash \bigvee_{C \in \mathcal{F}} \varphi_{C} \quad \text { and } \quad \mathcal{V}^{\prime}(A) \vdash \bigwedge_{C \neq C^{\prime} \in \mathcal{F}} \neg\left(\varphi_{C} \wedge \varphi_{C^{\prime}}\right)
$$

Hence, if $\sigma$ is a strictly convergent analytic $\mathcal{A}$-structure on the field $K$, there will be exactly one $C \in \mathcal{F}$ such that $K^{\circ} \models \varphi_{C}$ under the interpretation provided by $\sigma$. As in the separated case, we call such $C$ compatible with $\sigma$.

In the strictly convergent case $\pi \neq 1$ we do not need Laurent rings in the formulation of the Strong Noetherian Property.

Theorem 4.3.10 (The Strictly Convergent Strong Noetherian Property, $\pi \neq 1$ ). Let $\left\{A_{m}\right\}$ be a strictly convergent Weierstrass system, let $m \leq m^{\prime}, \xi=\left(\xi_{1}, \ldots, \xi_{m}\right), \xi^{\prime}=$ $\left(\xi_{1}, \ldots, \xi_{m^{\prime}}\right), \xi^{\prime \prime}=\left(\xi_{m+1}, \ldots, \xi_{m^{\prime}}\right)$ and

$$
f=\sum_{\mu} \bar{f}_{\mu}(\xi)\left(\xi^{\prime \prime}\right)^{\mu} \in A_{m^{\prime}}
$$

with the $\bar{f}_{\mu}(\xi)$ in $A_{m}$. There is a (finite) system $\mathcal{F}$ of rings of A-fractions such that for each $C \in \mathcal{F}$ there is a finite set $J_{C}$ and functions $h_{\mu} \in C_{m}^{\circ}$ for $\mu \in J_{C}$ such that

$$
f=\sum_{\mu \in J_{C}} \bar{f}_{\mu}(\xi)\left(\xi^{\prime \prime}\right)^{\mu}\left(1+h_{\mu}\right)
$$

as an element of $C_{m^{\prime}}$.

Proof. This is proved similarly to the special case $n=0$ of Theorem 4.2 .15

\subsection{Examples of analytic structures}

(1) Definition 4.1.6 generalizes the notions of analytic structures in [vdD] and [CLR1]. Even more, if $A$ is Noetherian, complete and separated in its $I$-adic topology, and we take $A_{m, n}:=A\langle\xi\rangle[[\rho]]$, then the conditions (a)-(c) of Definition 4.1.3 are satisfied; (c) is immediate and (a) and (b) are shown in [CLR1]. (Only the subcase $I=0$ is not taken care of in [CLR1], but can be treated similarly.) (In [CLR1] only the case $C=A$ is treated, but the general case also follows easily from the Strong Noetherian Property of [CLR1]. Indeed, the stronger property of Remark 4.2.16 is satisfied in this example.)

(2) Definitions 4.1.6 and 4.3.6 generalize the notion of analytic structure in [DHM], since that is a special instance of the analytic structure of [vdD] and [CLR1]. To recall, take $F=\mathbb{Q}_{p}, T_{m}^{\circ}=\mathbb{Z}_{p}\langle\xi\rangle$ and $\mathcal{S}=\left\{T_{m}^{\circ}\right\}$, or equivalently take $A=\mathbb{Z}_{p}, I=p \mathbb{Z}_{p}, t=p$ and $\mathcal{A}=\left\{A_{m, 0}\right\}$, where $A_{m, 0}:=\mathbb{Z}_{p}\langle\xi\rangle$. In [DHM], $p$-adically closed fields with strictly 
convergent $\mathcal{S}$-structure are studied, in particular, these fields turn out to be elementarily equivalent to $\mathbb{Q}_{p}$ with subanalytic structure.

(3) Definitions 4.1 .6 and 4.3 .6 generalize the notion of analytic structure (implicit) in [LR1]. Let $K$ be a complete, rank one valued field and take $S_{m, n}^{\circ}=S_{m, n}^{\circ}(E, K)$, $\mathcal{S}=\left\{S_{m, n}^{\circ}\right\}$ as defined in [LR1], Definition 2.1.1. If $K^{\prime}$ is any complete field containing $K$ (or an algebraic extension of such a field) then $K^{\prime}$ has separated analytic $\mathcal{S}$-structure, with $\sigma$ defined naturally by the inclusion $K \subset K^{\prime}$. If $K^{*}$ is a (nonstandard) model of the theory of $K^{\prime}$ in the valued field language with function symbols for the elements of $\mathcal{S}$ then $K^{*}$ has separated analytic $\mathcal{S}$-structure, as shown in [LR3]. We also showed in [LR3] that if $K^{\prime}$ is a maximally complete field extending $K$ then $K^{\prime}$ has a separated analytic $\mathcal{S}$-structure. (Similar statements hold for strictly convergent analytic $\mathcal{S}$-structure).

In [LR2] we established that certain subrings $\mathcal{E}_{m, n}^{\circ}$ of the $S_{m, n}^{\circ}$, namely the elements $f$ of $S_{m, n}^{\circ}(E, K)$ such that $f$ and all its (Hasse) derivatives are existentially definable over $T_{m+n}(K)$, form a separated Weierstrass system. This was used in that paper to prove a quantifier simplification theorem ([LR2, Corollary 4.5]) for $K_{\text {alg }}^{\prime}$ in the language with function symbols for the elements of $\bigcup_{n} T_{n}(K)$. That quantifier simplification theorem (and extensions) thus follow from Theorem 4.5.15 below.

(4) We give another example which does not fall under the scope of previous papers. Let $F$ be a fixed maximally complete field with value group $\Gamma$ (see Section 2 above). In the equicharacteristic case let $E$ be a copy of the residue field in $F$ and in the mixed characteristic case let $E$ be a set of multiplicative representatives of $\widetilde{F}$ as in Section 2 . For a ring $B \subset F^{\circ}$ let $B_{0}=B \cap E$, and define

$$
B\langle\xi\rangle:=\left\{\sum_{g \in I} t^{g} p_{g}(\xi): t^{g} \in B, p_{g}(\xi) \in B_{0}[\xi] \text { and } I \subset \Gamma \text { is well ordered }\right\} .
$$

Let $\mathcal{B}$ be the family of subrings $B$ of $F^{\circ}$ with $E \subset B$ and $\operatorname{supp}(B)$ well ordered. Take $S_{m, n}^{\circ}(\mathcal{B})=\bigcup_{B \in \mathcal{B}} B\langle\xi\rangle[[\rho]]$ and $S_{m, n}(\mathcal{B}):=F \otimes_{F^{\circ}} S_{m, n}^{\circ}(\mathcal{B})$. Then we call $S_{m, n}(\mathcal{B})$ the full ring of separated power series over $F$ and $T_{m}(\mathcal{B}):=S_{m, 0}(\mathcal{B})$ the full ring of strictly convergent power series over $F$. The elements of $S_{m, n}(\mathcal{B})$ naturally define functions $\left(F_{\text {alg }}^{\circ}\right)^{m} \times\left(F_{\text {alg }}^{\circ}\right)^{n} \rightarrow F_{\text {alg }}$, where $F_{\text {alg }}$ is the algebraic closure of $F$, or its maximal completion (cf. [LR3, Section 5]). The family $\mathcal{S}:=\left\{S_{m, n}^{\circ}(\mathcal{B})\right\}$ is a strong Weierstrass system, yielding a separated analytic $\mathcal{S}$-structure on $F$, or on $F_{\text {alg. Indeed, most of the }}$ structure theorems that hold for the Tate rings in the classical affinoid case ([BGR] $)$ or the rings of separated power series in the quasi-affinoid case ([LR1]) will also hold for these rings, providing a basis for affinoid or quasi-affinoid algebra and geometry over maximally complete fields.

(5) See Theorems 4.5.7 and 4.5.11 below for further natural examples obtained by extending analytic structures by putting in constants from a model, resp. by going to algebraic extensions of a model. ("Model" meaning here field with analytic structure.)

(6) The case of trivially valued field $F$ does not fall under the scope of the previously mentioned papers (as far as we know), but might be interesting for the study of tame analytic integrals (generalizing [D3], [Pas1]). When $F$ is a trivially valued field, the family $\left\{B_{m, n}\right\}$ with $B_{m, n}=F[\xi][[\rho]]$ is a strong separated Weierstrass system, since 
$F[\xi][[\rho]]$ equals $F[[\rho]]\langle\xi\rangle$, the $\rho$-adically strictly convergent power series in $\xi$ over $F[[\rho]]$ (cf. [LR1] or [CLR1]). The conditions of Definition 4.1.2 are immediate, as are the Weierstrass Division Theorems, (a) and (b) (see [LR1]). $B_{m, n}$ is Noetherian since a power series ring over a Noetherian ring is Noetherian. Property (c) is satisfied since $F[\xi][[\rho]]$ is the "full" power series ring. A field $K$ with analytic $\left\{B_{m, n}\right\}$-structure need not be trivially valued.

(7) (An example where $A$ is neither Noetherian, nor a valuation ring.) Let $L$ be a field and $A_{i}:=L\left[x_{1}, \ldots, x_{i}\right], I_{i}:=\left(x_{1}, \ldots, x_{i}\right) A_{i}$ and $A:=\bigcup_{i} A_{i}, I:=\bigcup_{i} I_{i}$. Then $A_{m, n}:=\bigcup_{i} A_{i}\langle\xi\rangle[[\rho]]$ is a (strong) separated Weierstrass system, though $A$ is neither Noetherian, nor a valuation ring.

(8) A simple compactness type argument shows that there is a strong Weierstrass system $\mathcal{A}^{\prime}=\left\{A_{m, n}^{\prime}\right\}$ with all the $A_{m, n}^{\prime} \subset \mathbb{Z}[[t]]\langle\xi\rangle[[\rho]]$ countable. This example is not covered by the treatments of the previous papers.

(9) We construct a separated Weierstrass system which is not strong (i.e. not satisfying Definition 5.6.1 (cf. Example 3.3(7)). Let $B_{m, n}^{\prime}$ be the algebraic closure of $\mathbb{Q}[\xi, \rho]$ in $\mathbb{Q}[\xi][[\rho]]$ (cf. Example (6).) Then the family $\left\{B_{m, n}^{\prime}\right\}$ is a separated Weierstrass system. Namely, the conditions of Definition 4.1.2 are easy, Weierstrass Division (axioms (a) and (b)) can be done with algebraic data, and condition (c) follows from axioms (a) and (b) in this case with $F=\mathbb{Q}$ by a proof similar to the proof of Theorem 4.2.15. However, the family $\left\{B_{m, n}^{\prime}\right\}$ does not satisfy condition 5.6.1 (v), as can be seen as follows. Let $f\left(\xi_{1}, \xi_{2}\right)$ be an algebraic power series whose diagonal is not algebraic (see for example $3.3(7)$ ). If

$$
f\left(\rho \xi_{1}, \rho \xi_{2}\right)=f_{1}\left(\rho, \xi_{1}\right)+f_{2}\left(\rho, \xi_{2}\right) \bmod \left(\xi_{1} \xi_{2}-1\right),
$$

then

$$
f_{1}(0, \rho)+f_{2}(0, \rho)=g\left(\rho^{2}\right),
$$

where $g$ is the diagonal of $f$, and where $\rho$ is a single variable. But $g\left(\rho^{2}\right)$ is not algebraic.

(10) Let $K$ be a complete valued field, and let $K\langle\langle\xi\rangle\rangle$ be the ring of overconvergent power series over $K$ (i.e. the elements of $K\langle\xi\rangle$ with radius of convergence $>1$ ). Let $\mathcal{B}$ be a family of quasi-Noetherian subrings of $K^{\circ}$ as in Definition 2.1.1 of [LR1]. For $\gamma>1$ let

$$
\begin{aligned}
B\langle\xi\rangle[[\rho]]^{(\gamma)} & :=\left\{\sum_{\mu, \nu} a_{\mu \nu}(\xi)^{\mu}(\rho)^{\nu}: \exists k \in \mathbb{N}\left(|\mu|>k \rightarrow\left|a_{\mu \nu}\right|<\gamma^{-|\mu|} \text { or }|\nu|>(\gamma-1)|\mu|\right)\right\} \\
& \subset B\langle\xi\rangle[[\rho]]
\end{aligned}
$$

be the subring of $\gamma$-overconvergent power series with coefficients from B. Define

$$
S_{m, n}^{\circ}(E, K)^{\text {over }}:=\bigcup_{\substack{B \in \mathcal{B} \\ \gamma>1}} B\langle\xi\rangle[[\rho]]^{(\gamma)} .
$$

Then $\mathcal{S}^{\text {over }}:=\left\{S_{m, n}^{\circ}(E, K)^{\text {over }}\right\}$ is a separated Weierstrass system, and $K$ has separated analytic $\mathcal{S}^{\text {over }}$-structure and strictly convergent analytic $\{K\langle\langle\xi\rangle\rangle\}$-structure (Definition 
4.3.6(ii)). Hence some of the results of $[\mathrm{S}]$ fit into our context. We leave the verification that $\mathcal{S}^{\text {over }}$ is a (strong) separated Weierstrass system to the reader. This construction can be extended in various nonstandard directions, for example, to maximally complete fields (cf. example (4) above).

(11) If $\mathcal{A}$ is a (strong) Weierstrass system, and $J \subset A$ is an ideal (with $(I, J) \subset$ $A$ proper), then $\mathcal{A} / J:=\left\{A_{m, n} / J A_{m, n}\right\}$ is also a (strong) Weierstrass system, with $(A / J)^{\circ}=(I, J) / J$. The conditions in Definitions 4.1.2 and 4.1.5 are immediate, as are the conditions of Definition 5.6.1 if $\mathcal{A}$ is strong. The conditions of 4.1 .3 (Weierstrass Division) are not quite immediate as we may have $f \in A_{m, n} / J A_{m, n}$ regular in $\xi_{m}$ (respectively $\rho_{n}$ ) of degree $s$, and $f \equiv F \bmod J A_{m, n}, F \in A_{m, n}$ without $F$ being regular in $\xi_{m}$ (respectively $\rho_{n}$ ) of degree $s$. However, as in the proof of Lemma 4.1.12, we can modify $F$ to find a $G \in A_{m, n}$ with $f \equiv G$ and $G$ regular in $\xi_{m}$ (respectively $\rho_{n}$ ) of degree $s$.

(12) Further important examples are provided by Definition 4.5.6 (see Theorem 4.5.7) and Theorem 4.5.11

(13) Every Henselian valued field carries an (algebraic) analytic structure all the functions of which are definable, see Section 4.6 . Hence the (algebraic) theory of Henselian valued fields is included in our formalism of (Henselian) fields with analytic structure.

In examples (1) and (2) of strictly convergent analytic structures $(\pi \neq 1)$, the strictly convergent analytic structure gives the same family of analytic functions as a natural separated analytic structure. Hence, all results on separated analytic structures apply. We do not know any example where this is not the case. We will for this reason from Section 5 on focus on separated analytic structures.

\subsection{Properties of analytic structures}

In this subsection we develop some of the basic properties of separated and strictly convergent analytic structures.

Remark 4.5.1. If $K$ is trivially valued (i.e. $\left.K^{\circ \circ}=(0)\right)$ then $I \subset \operatorname{ker} \sigma_{0}$ and the analytic structure collapses to the usual algebraic structure given by polynomials. See Example $4.4(11)$. The domain of the $\rho$ variables is $\{0\}$ if $K^{\circ \circ}=(0)$.

Remark 4.5.2. Considering $A_{m, n} \subset A[[\xi, \rho]]$, we do not know a priori that "composition" or "substitution" by elements of $A_{M, N}$ for $\xi$-variables and elements of $\left(A_{M, N}\right)^{\circ}$ for $\rho$-variables in the rings $A_{m, n}$ makes sense. However, properties (a) and (b) (Weierstrass Division) of Definition 4.1.3 (or property (a) of Definition 4.3.3 allow us to define composition in these rings. For example, dividing $f\left(\xi_{1}\right)$ by $\xi_{1}-g\left(\xi_{2}\right)$ gives $f\left(\xi_{1}\right)=$ $Q \cdot\left(\xi_{1}-g\left(\xi_{2}\right)\right)+h\left(\xi_{2}\right)$ and we can define $f\left(g\left(\xi_{2}\right)\right)$ to be $h\left(\xi_{2}\right)$. Definition 4.1 .3 (or Definition 4.3.3 guarantees that this actually is composition on the "top slice". In all the standard examples (Examples 4.4 composition defined in this way is actually power series composition. In a field with analytic structure (i.e. after applying $\sigma$ ) this "defined" composition becomes actual composition. In the above example, $\sigma\left(f\left(\xi_{1}\right)\right)=$ $\sigma(Q)\left(\xi_{1}-\sigma\left(g\left(\xi_{2}\right)\right)\right)+\sigma\left(h\left(\xi_{2}\right)\right)$, so $\sigma(h)=\sigma(f) \circ \sigma(g)$. 
Proposition 4.5.3. Analytic $\mathcal{A}$-structures preserve composition. More precisely, if $\mathcal{A}=$ $\left\{A_{m, n}\right\}$ and $f \in A_{m, n}, \alpha_{1}, \ldots, \alpha_{m} \in A_{M, N}, \beta_{1}, \ldots, \beta_{n} \in\left(A_{M, N}\right)^{\circ}$, then $g:=f(\alpha, \beta)$ is in $A_{M, N}$ and $\sigma(g)=(\sigma(f))(\sigma(\alpha), \sigma(\beta))$.

Proof. As in [CLR1, Proposition 2.8], and the above remark, this follows from Weierstrass Division.

Proposition 4.5.4. In a nontrivially valued field with analytic $\mathcal{A}$-structure, the image of a power series is the zero function if and only if the image of each of its coefficients is zero. More precisely, for $K$ a nontrivially valued field:

(i) Let $\sigma$ be a separated analytic $\mathcal{A}$-structure on $K$. Then

$$
\operatorname{ker} \sigma_{m, n}=\left\{\sum_{\mu, \nu} a_{\mu, \nu} \xi^{\mu} \rho^{\nu} \in A_{m, n}: a_{\mu, \nu} \in \operatorname{ker} \sigma_{0,0}\right\} .
$$

Furthermore, with the notation of Definition $4.1 .2(\mathrm{v})$, if

$$
f\left(\xi^{\prime}, \rho^{\prime}\right)=\sum_{\mu, v} \bar{f}_{\mu \nu}(\xi, \rho)\left(\xi^{\prime \prime}\right)^{\mu}\left(\rho^{\prime \prime}\right)^{v}
$$

and $a \in\left(K^{\circ}\right)^{m}, b \in\left(K^{\circ \circ}\right)^{n}$, then the function

$$
\left(K^{\circ}\right)^{m^{\prime}-m} \times\left(K^{\circ \circ}\right)^{n^{\prime}-n} \rightarrow K^{\circ}:(c, d) \mapsto \sigma_{m^{\prime}, n^{\prime}}(f)(a, c, b, d)
$$

is the zero function exactly when $\sigma_{m, n}\left(\bar{f}_{\mu \nu}\right)(a, b)=0$ for all $\mu, \nu$.

(ii) $(\pi \neq 1)$ Let $\sigma$ be a strictly convergent analytic $\mathcal{A}$-structure on $K$. Then

$$
\operatorname{ker} \sigma_{m}=\left\{\sum_{\mu} a_{\mu} \xi^{\mu} \in A_{m}: a_{\mu} \in \operatorname{ker} \sigma_{0}\right\} .
$$

Furthermore, with the notation of Definition 4.3.1 (v), if $f\left(\xi^{\prime}\right)=\sum_{\mu} \bar{f}_{\mu}(\xi)\left(\xi^{\prime \prime}\right)^{\mu}$ and $a \in\left(K^{\circ}\right)^{m}$, then the function

$$
\left(K^{\circ}\right)^{m^{\prime}-m} \rightarrow K^{\circ}: c \mapsto \sigma_{m^{\prime}}(f)(a, c)
$$

is the zero function exactly when $\sigma_{m}\left(\bar{f}_{\mu}\right)(a)=0$ for all $\mu$.

Proof. This follows easily from the Strong Noetherian Property and Weierstrass Preparation. Case (i) in the case that $K$ is nontrivially valued is given in detail in [CLR1, Proposition 2.10].

Remark 4.5.5. Proposition 4.5.4 does not always hold when $K$ is trivially valued. Namely, in case (i), the function $\sigma\left(\rho_{1}\right)$ is the zero function. In case (ii), if $K$ is a finite field there is a nonzero monic polynomial $p\left(\xi_{1}\right) \in A_{1}$ such that $\sigma(p)$ is the zero function. There is no problem in the construction of Remark 4.5 .8 below however. 
When considering a particular field $K$ with analytic $\mathcal{A}$-structure, it is no loss of generality to assume that ker $\sigma_{0,0}=(0)$ (see Example 4.4.11)). Indeed we can replace $A$ by $A / \operatorname{ker} \sigma_{0,0}$ to get an equivalent analytic structure on $K$ with this property. In the case that ker $\sigma_{0,0}=(0)$ we may consider $A_{0,0}=A$ to be a subring of $K^{\circ}$. It is convenient to extend the Weierstrass system by suitably adjoining the elements of $K$ to $A$ :

Definition 4.5.6 (Extension of parameters). (i) Let $\mathcal{A}=\left\{A_{m, n}\right\}$ be a separated Weierstrass system and let $K$ be a nontrivially valued valued field with analytic $\mathcal{A}$-structure $\left\{\sigma_{m, n}\right\}$. Assume that ker $\sigma_{0,0}=(0)$, so we may consider $A$ as a subring of $K^{\circ}$ and $A_{m, n}$ as a subring of $K^{\circ}[[\xi, \rho]]$. With the notation of Definition 4.1.2(v) and with $M=m^{\prime}-m$, $N=n^{\prime}-n$, if $f=\sum_{\mu, \nu} \bar{f}_{\mu \nu}(\xi, \rho)\left(\xi^{\prime \prime}\right)^{\mu}\left(\rho^{\prime \prime}\right)^{\nu}, a \in\left(K^{\circ}\right)^{m}$ and $b \in\left(K^{\circ \circ}\right)^{n}$, we write $f\left(a, \xi^{\prime \prime}, b, \rho^{\prime \prime}\right)$ for the power series

$$
\sum_{\mu, v} \sigma_{m, n}\left(\bar{f}_{\mu \nu}\right)(a, b)\left(\xi^{\prime \prime}\right)^{\mu}\left(\rho^{\prime \prime}\right)^{v} \quad \text { in } K^{\circ}\left[\left[\xi^{\prime \prime}, \rho^{\prime \prime}\right]\right] .
$$

Then by Proposition 4.5 .4 the function

$$
\left(K^{\circ}\right)^{M} \times\left(K^{\circ \circ}\right)^{N} \rightarrow K^{\circ}:(c, d) \mapsto(\sigma f)(a, c, b, d)
$$

only depends on the power series $f\left(a, \xi^{\prime \prime}, b, \rho^{\prime \prime}\right)$ and we denote this function by

$$
\sigma_{M, N}^{\prime}\left(f\left(a, \xi^{\prime \prime}, b, \rho^{\prime \prime}\right)\right) \text {. }
$$

Define the subring $A_{M, N}(K)$ of $K^{\circ}\left[\left[\xi^{\prime \prime}, \rho^{\prime \prime}\right]\right]$ by

$$
A_{M, N}(K):=\bigcup_{m, n \in \mathbb{N}}\left\{f\left(a, \xi^{\prime \prime}, b, \rho^{\prime \prime}\right): f \in A_{m^{\prime}, n^{\prime}}, a \in\left(K^{\circ}\right)^{m}, b \in\left(K^{\circ \circ}\right)^{n}\right\},
$$

and define

$$
\mathcal{A}(K):=\left\{A_{M, N}(K)\right\}_{M, N} .
$$

Then, $\sigma_{M, N}^{\prime}$ is a homomorphism from $A_{M, N}(K)$ to the ring of functions $\left(K^{\circ}\right)^{M} \times\left(K^{\circ \circ}\right)^{N}$ $\rightarrow K^{\circ}$, for each $M, N$.

(ii) $(\pi \neq 1)$ Let $\mathcal{A}=\left\{A_{m}\right\}$ be a strictly convergent Weierstrass system and assume that $K$ has analytic $\mathcal{A}$-structure $\left\{\sigma_{m}\right\}$ and that $\operatorname{ker} \sigma_{0}=(0)$ so $A=A_{0} \subset K^{\circ}$. With the notation of Definition 4.3.1 (v) and with $M=m^{\prime}-m$, if $f=\sum_{\mu} \bar{f}_{\mu}(\xi)\left(\xi^{\prime \prime}\right)^{\mu}$ and $a \in\left(K^{\circ}\right)^{m}$, define the power series $f\left(a, \xi^{\prime \prime}\right)$ as $\sum_{\mu} \sigma_{m}\left(\bar{f}_{\mu}\right)(a)\left(\xi^{\prime \prime}\right)^{\mu} \in K^{\circ}\left[\left[\xi^{\prime \prime}\right]\right]$. Then by Proposition 4.5.4 the function $\sigma_{M}^{\prime}\left(f\left(a, \xi^{\prime \prime}\right)\right)$ defined by

$$
\left(K^{\circ}\right)^{M} \rightarrow K^{\circ}: c \mapsto \sigma_{m^{\prime}}(f)(a, c)
$$

only depends on the power series $f\left(a, \xi^{\prime \prime}\right)$. Define

$$
\begin{aligned}
A_{M}(K) & :=\bigcup_{m \in \mathbb{N}}\left\{f\left(a, \xi^{\prime \prime}\right): f \in A_{m+M}, a \in\left(K^{\circ}\right)^{m}\right\} \subset K^{\circ}\left[\left[\xi^{\prime \prime}\right]\right], \\
\mathcal{A}(K) & :=\left\{A_{M}(K)\right\} .
\end{aligned}
$$

Then $\sigma_{M}^{\prime}$ is a homomorphism from $A_{M}(K)$ to the ring of functions $\left(K^{\circ}\right)^{M} \rightarrow K^{\circ}$. 
We have

Theorem 4.5.7. (i) Let $K$ and $\mathcal{A}$ be as in Definition 4.5.6(i). Then $\mathcal{A}(K)$ is a separated Weierstrass system over $\left(K^{\circ}, K^{\circ \circ}\right)$ and $K$ has separated analytic $\mathcal{A}(K)$-structure via the homomorphisms $\left\{\sigma_{M, N}^{\prime}\right\}$. The family $\left\{A_{M, 0}(K)\right\}$ is a strictly convergent Weierstrass system and $\left\{\sigma_{M, 0}^{\prime}\right\}$ provides $K$ with a strictly convergent analytic structure as described in Definition 4.3.6 (ii). (No rings of $K^{\circ}$-fractions are needed as $K^{\circ}$ is a valuation ring.) If $\mathcal{A}$ is a strong Weierstrass system, so is $\mathcal{A}(K)$.

(ii) Let $K$ and $\mathcal{A}$ be as in Definition 4.5.6(ii). Then $\mathcal{A}(K)$ is a strictly convergent Weierstrass system and $K$ has strictly convergent analytic $\mathcal{A}(K)$-structure via the homomorphisms $\left\{\sigma_{M}^{\prime}\right\}$. If $\mathcal{A}$ is a strong Weierstrass system, so is $\mathcal{A}(K)$.

Proof. The various properties for $\mathcal{A}(K)$ follow using the Weierstrass Division Theorem and the Strong Noetherian Property and the corresponding properties for $\mathcal{A}$. (The case when $A_{m, n}=A\langle\xi\rangle[[\rho]]$, with $A$ Noetherian and complete in its $I$-adic topology is given in [CLR1, Lemma-Definition 2.12].)

Remark 4.5.8 (More general constants). Let $K$ and $\mathcal{A}$ be as in Definition 4.5.6 (i), and use its notation. For $K^{\prime}$ any subfield of $K$, one can define

$$
A_{M, N}\left(K^{\prime}\right):=\bigcup_{m, n \in \mathbb{N}}\left\{f\left(a, \xi^{\prime \prime}, b, \rho^{\prime \prime}\right): f \in A_{m^{\prime}, n^{\prime}}, a \in\left(K^{\prime \circ}\right)^{m}, b \in\left(K^{\prime \circ}\right)^{n}\right\} .
$$

As in Theorem 4.5.7 if $K$ has analytic $\mathcal{A}$-structure, then it has analytic $\mathcal{A}\left(K^{\prime}\right)$ structure.

We restate the special case $m=0$ or $n=0$ of the Strong Noetherian Property (Theo$\operatorname{rem} 4.2 .15$ for $\mathcal{A}(K)$, that we will need in Section 5 .

Corollary 4.5.9. Let $\mathcal{A}=\left\{A_{m, n}\right\}$ be a separated Weierstrass system and let nontrivially valued field $K$ have separated analytic $\mathcal{A}$-structure $\sigma$ with $\operatorname{ker}\left(\sigma_{0,0}\right)=(0)$. If $f \in A_{m+M, n+N}(K)$ and $m=0$ or $n=0$, we can write

$$
f=\sum_{(\mu, \nu) \in J} \bar{f}_{\mu \nu}(\xi, \rho)\left(\xi^{\prime \prime}\right)^{\mu}\left(\rho^{\prime \prime}\right)^{\nu}\left(1+g_{\mu \nu}\right)
$$

where the $g_{\mu \nu}$ are in $A_{m+M, n+N}^{\circ}$ and $J \subset \mathbb{N}^{M+N}$ is a finite set.

We restate some results from [CLR1] whose proofs extend without difficulty to our current more general setting.

\section{Proposition 4.5.10 (Proposition 2.17 of [CLR1]).}

(i) Let $\mathcal{A}$ be a separated Weierstrass system and let $K$ be a valued field with separated analytic $\mathcal{A}$-structure. Then $K^{\circ}$ is a Henselian valuation ring.

(ii) $(\pi \neq 1)$ Let $\mathcal{A}$ be a strictly convergent Weierstrass system and let $K$ be a valued field with strictly convergent analytic $\mathcal{A}$-structure. Then $K^{\circ}$ is a Henselian valuation ring.

The following theorem permits us to work over any algebraic extension of the domain of an analytic $\mathcal{A}$-structure. Its proof is the same as that of Theorem 2.18 of [CLR1]. 
Theorem 4.5.11. Let $K$ be a valued field and let $K^{\prime}$ be an algebraic extension of $K$.

(i) Let $\mathcal{A}$ be a separated Weierstrass system. Suppose that $K$ has a separated analytic $\mathcal{A}$-structure $\sigma=\left\{\sigma_{m, n}\right\}$. Then there is a unique extension of $\sigma$ to a separated analytic $\mathcal{A}$-structure $\tau$ on $K^{\prime}$.

(ii) $(\pi \neq 1)$ Let $\mathcal{A}$ be a strictly convergent Weierstrass system. Suppose that $K$ has a strictly convergent analytic $\mathcal{A}$-structure and that $\sigma(\pi)$ remains prime in $\left(K^{\prime}\right)^{\circ}$. Then there is a unique extension of $\sigma$ to a strictly convergent analytic $\mathcal{A}$-structure $\tau$ on $K^{\prime}$.

Remark 4.5.12. (i) Let $C$ be a ring of $\mathcal{A}$-fractions. Let $\sigma$ be a separated analytic $\mathcal{A}$ structure on $K$ and let $B$ be a Laurent ring over $C_{m, n}$ with defining formula $\varphi_{B}$. Then the formula $\varphi_{B}$ defines in a natural way a subset $\mathcal{U}_{\varphi_{B}}$ of $\left(K_{\mathrm{alg}}^{\circ}\right)^{m} \times\left(K_{\mathrm{alg}}^{\circ \circ}\right)^{n}$ by Theorem 4.5.11. If the set $\mathcal{U}_{\varphi_{B}}$ is nonempty, then it is called a Laurent subdomain in the terminology of [BGR, Definition 7.2.3.2], and a $K_{\mathrm{alg}}^{\circ}$-subdomain in [LR1, Definition 5.3.3]. Moreover, $B$ defines in a natural way a ring of (analytic) functions on $U_{\varphi_{B}}$, via $\sigma$. We will not study these rings of functions in detail.

(ii) Let $\sigma$ be a separated analytic $\mathcal{A}$-structure on $K$ and $C$ a ring of $\mathcal{A}$-fractions which is compatible with $\sigma$. Let $B$ be a Laurent ring over $C_{m, n}$ with a defining formula $\varphi_{B}$. Then $\mathcal{U}_{\varphi_{B}}$ as in (i) is nonempty if and only if $\varphi_{B}$ can be obtained inductively as in Definition 4.2 .8 such that for each step of type (ii) there exists $x$ in $\left(K_{\text {alg }}^{\circ}\right)^{m+M} \times\left(K_{\text {alg }}^{\circ \circ}\right)^{n+N}$ with $|\sigma(f)(x)| \geq 1$ and for each step of type (iii) there exists $x$ in $\left(K_{\text {alg }}^{\circ}\right)^{m+M} \times\left(K_{\text {alg }}^{\circ}\right)^{n+N}$ with $|\sigma(f)(x)|<1$. We will not use this property.

Remark 4.5.13. The conclusion of Proposition 4.5 .10 (ii), and hence also the conclusions of Theorem 4.5.11 (ii), can be false without the assumption that ord $\left(K^{\circ \circ}\right)$ has minimal element ord $\left(\sigma_{0}(b)\right)$. To see this consider $A_{m}=T_{m}^{\circ}\left(\mathbb{C}_{p}\right)$, the strictly convergent power series of Gauss norm $\leq 1$ over $\mathbb{C}_{p}$, the completion of the algebraic closure of $\mathbb{Q}_{p}$. Let $\mathbb{C}_{p}^{*}$ be a nonprincipal ultrapower of $\mathbb{C}_{p}$, and let $b \in \mathbb{C}_{p}^{*}$ satisfy $1-1 / n<|b|<1$ for all $n \in \mathbb{N}$. Let $K$ be the smallest substructure of $\mathbb{C}_{p}^{*}$ containing $b$ and closed under $+, \cdot, \cdot(\cdot)^{-1}$ and all the functions of $\bigcup_{m} A_{m}$. Then, by definition, $K$ has analytic $\left\{A_{m}\right\}$-structure in the sense that the elements of $A_{m}$ define functions on $K$ in a natural way. Let $p^{1 / \infty} \subsetneq K^{\circ \circ}$ be the ideal in $K^{\circ}$ generated by $\left\{p^{1 / n}: n \in \mathbb{N}\right\}$. One can see by induction on terms that $K^{\circ} / p^{1 / \infty} K^{\circ}=\left\{f(b) / g(b): f, g \in \overline{\mathbb{F}}_{p}[b]\right.$ and $\left.g(0) \neq 0\right\}$. Here $\overline{\mathbb{F}}_{p}$ is the algebraic closure of the $p$-element field. $K^{\circ}$ is not Henselian: consider $f(x)=1-x-b x^{2}$, which is regular in $x$ of degree 1 but has no zero in $K^{\circ}$. Indeed it has no zero in $K^{\circ} / p^{1 / \infty} K^{\circ}$.

Definition 4.5.14. Let $\mathcal{A}=\left\{A_{m, n}\right\}$ be a separated or strictly convergent Weierstrass system. Let $\mathcal{L}=\left\langle 0,1,+, \cdot,{ }^{-1}, \overline{,},|\cdot|\right\rangle$ be the language of valued fields (the symbol ${ }^{-}$denotes multiplication on the value group.) Let $\mathcal{L}_{\mathcal{A}}$ be $\mathcal{L}$ augmented with function symbols for the elements of $\bigcup_{m, n} A_{m, n}$. We call $\mathcal{L}_{\mathcal{A}}$ the language of valued fields with analytic $\mathcal{A}$-structure. Let $K$ be a valued field with analytic $\mathcal{A}$-structure. Interpret $f \in A_{m, n}$ as $\sigma(f):\left(K^{\circ}\right)^{m} \times\left(K^{\circ \circ}\right)^{n} \rightarrow K^{\circ}$, extended by zero outside its domain, so $K$ becomes an

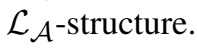

The following theorem extends [LL2, Theorem 3.8.2], [LR2, Theorem 4.2] and [LR3, Theorem 4.2]. 
Theorem 4.5.15 (Quantifier Elimination). Let $K$ be an algebraically closed valued field with separated analytic $\mathcal{A}$-structure. Let $\mathcal{L}_{\mathcal{A}}$ be as above. Then $K$ admits elimination of quantifiers in $\mathcal{L}_{\mathcal{A}}$.

Proof. The proofs of the above cited theorems, which are based on Weierstrass Division and the Strong Noetherian Property, work with very minor modifications.

Note that Theorem 4.5.15 does not impose any condition on the characteristic of $K$. (In fact, everything up to the end of Section 5 is for all characteristics.) In Section 6 , Theorem 6.3.7. we will give a quantifier elimination statement in the case that $K$ has characteristic zero but is not necessarily algebraically closed.

\subsection{The algebraic case}

In this subsection we show that every Henselian field carries a definable (algebraic) analytic structure, so our theory of Henselian fields with analytic structure contains the first order theory of Henselian fields as a special case. Along the way we establish some results about algebraic strictly convergent and separated power series over a discretely valued Henselian field that may be of independent interest. The results of this section are not used in the rest of the paper.

Let $R$ be a field or an excellent Henselian discrete valuation ring, with prime $\mathfrak{p}$. Let $\xi=\left(\xi_{1}, \ldots, \xi_{m}\right)$. Let $\widehat{R}$ denote the $\mathfrak{p}$-adic completion of $R$ and $\widehat{R}\langle\xi\rangle$ the ring of strictly convergent power series in $\xi$ with coefficients from $\widehat{R}$. Let $R\langle\xi\rangle_{\text {alg }}$ denote the algebraic closure of $R[\xi]$ in $\widehat{R}\langle\xi\rangle$, i.e. all the elements of $\widehat{R}\langle\xi\rangle$ that are algebraic over $R[\xi]$. (The condition of excellence for $R$ is the same as assuming that $\widehat{R}$ is separable over $R$, i.e. if $y_{1}, \ldots, y_{n} \in \widehat{R}$ then the field extension $Q\left(R\left[y_{1}, \ldots, y_{n}\right]\right)$ over $Q(R)$ has a separating transcendence base. Here $Q(A)$ denotes the quotient field of $A$.)

Theorem 4.6.1 (Artin Approximation for $R\langle\xi\rangle_{\text {alg }}$ ). Let $\eta=\left(\eta_{1}, \ldots, \eta_{N}\right)$, let $F_{i}(\xi, \eta) \in$ $R[\xi, \eta]$ for $i=1, \ldots, d$ be a finite set of polynomials, and let $c \in \mathbb{N}$ and $\bar{\eta}(\xi) \in(\widehat{R}\langle\xi\rangle)^{M}$ satisfy $F_{i}(\xi, \bar{\eta}(\xi))=0$ for $i=1, \ldots, d$. Then there exist $\widetilde{\eta}(\xi) \in\left(R\langle\xi\rangle_{\text {alg }}\right)^{M}$ satisfying $F_{i}(\xi, \widetilde{\eta}(\xi))=0$ for $i=1, \ldots, d$ and $\widetilde{\eta}(\xi) \equiv \bar{\eta}(\xi)$ mod $\mathfrak{p}^{c}$. Moreover, there are $\widetilde{\zeta}_{1}, \ldots, \widetilde{\zeta}_{n} \in R\langle\xi\rangle_{\text {alg }}$ such that $R\left[\xi, \widetilde{\eta}, \widetilde{\zeta}_{1}, \ldots, \widetilde{\zeta}_{n}\right]$ is a Henselian extension of $R[\xi]$, i.e. the Jacobian of this extension is a unit-there are $G_{j} \in R[\xi, \eta, \zeta], j=1, \ldots, N+n$, such that $\left(\frac{\partial G}{\partial \eta \partial \zeta}\right)(\widetilde{\eta}, \widetilde{\zeta})$ is a unit.

Proof. The proof is the same as that of $[\overline{\mathrm{Ar}}]$ in the local case, except that one uses the strictly convergent Weierstrass Preparation and Division Theorems instead of the local versions. For an elementary exposition of the Néron desingularization see [DL1, Section 3]. (Alternatively, one could follow the slightly different proof given in [Bo].)

The quasi-affinoid or separated case is more complicated. First we introduce some notation. Let $\xi=\left(\xi_{1}, \ldots, \xi_{m}\right), \eta=\left(\eta_{1}, \ldots, \eta_{M}\right), \rho=\left(\rho_{1}, \ldots, \rho_{n}\right)$ and $\lambda=\left(\lambda_{1}, \ldots, \lambda_{N}\right)$,

$$
S_{m, n}^{\circ}(\widehat{R}):=\widehat{R}\langle\xi\rangle[[\rho]]
$$

and $S_{m, n}^{\circ}(R)_{\text {alg }}$ is the algebraic closure of $R[\xi, \rho]$ in $S_{m, n}^{\circ}(\widehat{R})$. 
Definition 4.6.2. An algebraic Laurent domain (in $\left(F_{\mathrm{alg}}^{\circ}\right)^{m} \times\left(F_{\mathrm{alg}}^{\circ \circ}\right)^{n}$, where $F$ is the quotient field of $R$ or $\widehat{R}$ ) is a domain defined inductively by a sequence of inequalities of the form $\left|p_{i}(\xi)\right| \square_{i} 1$ where each $p_{i}$ is a polynomial with $\left\|p_{i}\right\|=1$ and each $\square_{i} \in\{\geq,<\}$. For algebraic Laurent domains $\mathcal{U}$ we define the ring of $\widehat{R}$-analytic functions $\mathcal{O}_{\widehat{R}}(\mathcal{U})$ on $\mathcal{U}$ inductively as follows. If $\mathcal{O}_{\widehat{R}}(\mathcal{U})=S_{m^{\prime}, n^{\prime}}^{\circ}(\widehat{R}) / I$ and $p(\xi)$ is a polynomial with $\|p\|=1$ and

$$
\mathcal{U}^{\prime}:=\mathcal{U} \cap\{(\xi, \rho):|p(\xi)| \geq 1\}, \quad \mathcal{U}^{\prime \prime}:=\mathcal{U} \cap\{(\xi, \rho):|p(\xi)|<1\}
$$

then

$$
\begin{aligned}
\mathcal{O}_{\widehat{R}}\left(\mathcal{U}^{\prime}\right):=S_{m^{\prime}+1, n^{\prime}}^{\circ}(\widehat{R}) /\left(I, p(\xi) \xi_{m^{\prime}+1}-1\right), & \mathcal{O}_{\widehat{R}}^{\circ}\left(\mathcal{U}^{\prime}\right):=\left(\mathfrak{p}, \rho_{1}, \ldots, \rho_{n^{\prime}}\right) \mathcal{O}_{\widehat{R}}\left(\mathcal{U}^{\prime}\right) \\
\mathcal{O}_{\widehat{R}}\left(\mathcal{U}^{\prime \prime}\right):=S_{m^{\prime}, n^{\prime}+1}^{\circ}(\widehat{R}) /\left(I, p(\xi)-\rho_{n^{\prime}+1}\right), & \mathcal{O}_{\widehat{R}}^{\circ}\left(\mathcal{U}^{\prime \prime}\right):=\left(\mathfrak{p}, \rho_{1}, \ldots, \rho_{n^{\prime}+1}\right) \mathcal{O}_{\widehat{R}}\left(\mathcal{U}^{\prime \prime}\right)
\end{aligned}
$$

For $\mathcal{U}$ an algebraic Laurent domain we define the ring $\mathcal{O}_{R}(\mathcal{U})_{\text {alg }}$ of algebraic functions to be the algebraic closure of $R[\xi, \rho]$ in $\mathcal{O}_{\widehat{R}}(\mathcal{U})$, and

$$
\mathcal{O}_{R}^{\circ}(\mathcal{U})_{\text {alg }}:=\mathcal{O}_{R}(\mathcal{U})_{\text {alg }} \cap \mathcal{O}_{\widehat{R}}^{\circ}(\mathcal{U})
$$

A finite family $\left\{\mathcal{U}_{i}\right\}$ of algebraic Laurent domains is called covering if $\bigcup_{i} \mathcal{U}_{i}=\left(F_{\text {alg }}^{\circ}\right)^{m}$ $\times\left(F_{\text {alg }}^{\circ \circ}\right)^{n}$.

Theorem 4.6.3 (Artin Approximation for $S_{m, n}^{\circ}(R)_{\text {alg }}$ ). Let $F_{i}(\xi, \eta, \rho, \lambda) \in R[\xi, \eta, \rho, \lambda]$ for $i=1, \ldots, d$, let $c \in \mathbb{N}$ and suppose that $\bar{\eta}(\xi, \rho) \in\left(S_{m, n}^{\circ}(\widehat{R})\right)^{M}, \bar{\lambda}(\xi, \rho) \in$ $(\mathfrak{p}, \rho)\left(S_{m, n}^{\circ}(\widehat{R})\right)^{N}$ satisfy

$$
F_{i}(\xi, \rho, \bar{\eta}(\xi, \rho), \bar{\lambda}(\xi, \rho))=0
$$

for $i=1, \ldots, d$. Then there is a finite covering family $\left\{\mathcal{U}_{i}\right\}, i=1, \ldots$, e, of algebraic Laurent domains and for each $i$ there are

$$
\tilde{\eta}^{(i)}(\xi, \rho) \in\left(\mathcal{O}_{R}\left(\mathcal{U}_{i}\right)_{\mathrm{alg}}\right)^{M}, \quad \tilde{\lambda}^{(i)}(\xi, \rho) \in\left(\mathcal{O}_{R}^{\circ}\left(\mathcal{U}_{i}\right)_{\mathrm{alg}}\right)^{N}
$$

such that

$$
\begin{aligned}
\tilde{\eta}^{(i)}(\xi, \rho) & \equiv \bar{\eta}^{(i)}(\xi, \rho) \bmod (\mathfrak{p}, \rho)^{c}, \\
\tilde{\lambda}^{(i)}(\xi, \rho) & \equiv \bar{\lambda}^{(i)}(\xi, \rho) \bmod (\mathfrak{p}, \rho)^{c}, \\
F_{j}\left(\xi, \rho, \widetilde{\eta}^{(i)}, \widetilde{\lambda}^{(i)}\right) & =0 \quad \text { for } j=1, \ldots, d .
\end{aligned}
$$

Moreover, for each $i$ there are $\widetilde{\zeta}^{(i)}=\left(\widetilde{\zeta}_{1}^{(i)}, \ldots, \widetilde{\zeta}_{L}^{(i)}\right) \in\left(\mathcal{O}_{R}\left(\mathcal{U}_{i}\right)_{\mathrm{alg}}\right)^{L}$ and $\widetilde{\sigma}^{(i)}=$ $\left(\widetilde{\sigma}_{1}^{(i)}, \ldots, \widetilde{\sigma}_{L^{\prime}}^{(i)}\right) \in\left(\mathcal{O}_{R}^{\circ}\left(\mathcal{U}_{i}\right)_{\mathrm{alg}}\right)^{L^{\prime}}$ such that $\mathcal{O}_{R}\left(\mathcal{U}_{i}\right)_{\mathrm{alg}}[\tilde{\eta}, \tilde{\lambda}, \tilde{\zeta}, \tilde{\sigma}]$ is a Henselian extension of $\mathcal{O}_{R}\left(\mathcal{U}_{i}\right)_{\mathrm{alg}}$, i.e. the Jacobian of the extension is a unit-there are $G_{j} \in R[\xi, \eta, \lambda, \zeta, \sigma]$, $j=1, \ldots, M+N+L+L^{\prime}$, such that $\left(\frac{\partial G}{\partial \eta \partial \lambda \partial \zeta \partial \sigma}\right)(\tilde{\eta}, \widetilde{\zeta}, \tilde{\lambda}, \widetilde{\sigma})$ is a unit. 
Proof. The proof, including the Néron $\mathfrak{p}$-desingularization, is the same as in the affinoid case, except for the following complication. As in the affinoid case we reduce to the situation (using the notation of [ $[\mathrm{Ar}]$ ) that

$$
F(\xi, \rho, \bar{\eta}, \bar{\lambda})=0 \quad \text { and } \quad \bar{\delta}=\delta(\xi, \rho, \bar{\eta}, \bar{\lambda}) \not \equiv 0 \bmod \mathfrak{p}
$$

where $\delta$ is the determinant of a suitable minor of the Jacobian matrix. It may not be possible to make $\bar{\delta}$ regular by permissible changes of variables among the $\xi_{i}$ and among the $\rho_{j}$. However, $\bar{\delta}$ will be preregular (see Definition 4.2.12) of degree $\left(\mu_{0}, \nu_{0}\right)$, say. Writing

$$
\bar{\delta}(\xi, \rho)=\sum_{v} \bar{\delta}_{v}(\xi) \rho^{v}
$$

we break up into two algebraic Laurent domains as follows:

(i) $\mathcal{U}_{1}$ defined by $\left|\bar{\delta}_{\nu_{0}}(\xi)\right| \geq 1$, i.e. $\bar{\delta}_{\nu_{0}} \xi_{m+1}-1=0$,

(ii) $\mathcal{U}_{2}$ defined by $\left|\bar{\delta}_{\nu_{0}}(\xi)\right|<1$, i.e. $\bar{\delta}_{\nu_{0}}-\rho_{n+1}=0$.

Case (i). After Weierstrass changes of variables among the $\xi_{i}$ and among the $\rho_{j}$, we may assume that $\bar{\delta}_{\nu_{0}} \xi_{m+1}-1$ is regular in $\xi_{m+1}$ of degree $s$ say, and that $\xi_{m+1} \bar{\delta}$ is regular in $\rho_{n}$. We are thus reduced to seeking solutions in $\mathcal{O}_{R}\left(\mathcal{U}_{1}\right)_{\text {alg }}$ (which is a finite $S_{m, n}^{\circ}(R)_{\text {alg- }}$ algebra) with $\bar{\delta}$ regular in $\rho_{n}$ and we proceed as in the affinoid or local cases by induction

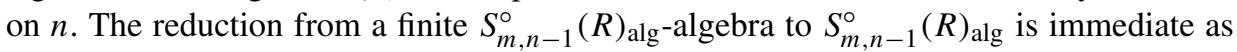
in $[\mathrm{Ar}]$.

Case (ii). After a Weierstrass change of variables among the $\xi_{i}$, we may assume that $\bar{\delta}_{\nu_{0}}-\rho_{n+1}$ is regular in $\xi_{m}$. Hence we are reduced to the case of a finite algebra over $S_{m-1, n+1}^{\circ}(R)_{\text {alg }}$, and hence to the case of $S_{m-1, n+1}^{\circ}(R)_{\text {alg }}$, and the proof follows by induction on the pairs $(m, n)$ ordered lexicographically. The case $m=0$ is like the affinoid case: $\bar{\delta}$ can be made regular in $\rho_{n}$ by a Weierstrass change of variables among the $\rho_{j}$.

Remark 4.6.4. The condition on the Jacobian in Theorem 4.6.3 (and Theorem 4.6.1) shows that all the algebraic power series (i.e. power series in $\mathcal{O}_{R}^{\circ}(\mathcal{U})_{\text {alg }}$ for $\mathcal{U}$ a Laurent domain) define functions on any Henselian field containing $R$ that are existentially definable in the language of Henselian fields.

Theorem 4.6.5. $S_{m, n}^{\circ}(R)_{\text {alg }}$ satisfies the two Weierstrass Preparation and Division Theorems (Definition 4.1.3. In other words, if $f, g \in S_{m, n}^{\circ}(R)_{\text {alg }}$ and $f$ is regular (in $\xi_{m}$ or $\left.\rho_{n}\right)$, then the associated Weierstrass data are algebraic.

Proof. First we prove that the data associated with Weierstrass Preparation applied to a regular algebraic power series are algebraic. Let $f(\xi, \rho) \in S_{m, n}^{\circ}(R)_{\text {alg }}$ be regular in $\xi_{m}$ of degree $s$ say. (The case that $f$ is regular in $\rho_{n}$ is similar.) Let $\xi^{\prime}=\left(\xi_{1}, \ldots, \xi_{m-1}\right)$. Then there is an $F(\xi, \rho, Y) \in R[\xi, \rho, Y] \backslash\{0\}$ such that $F(\xi, \rho, f(\xi, \rho))=0$. We may assume that $F(\xi, \rho, 0) \neq 0$. Let

$$
f(\xi, \rho)=U(\xi, \rho)\left[\xi_{m}^{s}+A_{s-1}\left(\xi^{\prime}, \rho\right) \xi_{m}^{s-1}+\cdots+A_{0}\left(\xi^{\prime}, \rho\right)\right]
$$


with the $A_{j}$ in $S_{m-1, n}^{\circ}(\widehat{R})$ and $U \in S_{m, n}^{\circ}(\widehat{R})$. Let the $\bar{\xi}_{m, j}$ be the zeros of $\xi_{m}^{s}+$ $A_{s-1}\left(\xi^{\prime}, \rho\right) \xi^{s-1}+\cdots+A_{0}\left(\xi^{\prime}, \rho\right)$ in the algebraic closure of the quotient field of $S_{m, n}^{\circ}(\widehat{R})$. Then the $\bar{\xi}_{m, j}$ all satisfy $F\left(\xi^{\prime}, \bar{\xi}_{m, j}, \rho, 0\right)=0$, and hence are algebraic. The $A_{i}\left(\xi^{\prime}, \rho\right)$ are symmetric functions of the $\bar{\xi}_{m, j}$ and hence are also algebraic, i.e. in $S_{m, n}^{\circ}(R)_{\text {alg. }}$. It follows immediately that $U(\xi, \rho)$ is also algebraic.

Next we show that the Weierstrass data associated with Weierstrass Division of an algebraic power series by a Weierstrass polynomial in $\xi_{m}$ are algebraic. Let $g \in S_{m, n}^{\circ}(R)_{\text {alg }}$, and $f=\xi_{m}^{s}+A_{s-1}\left(\xi^{\prime}, \rho\right) \xi^{s-1}+\cdots+A_{0}\left(\xi^{\prime}, \rho\right)=: W$ with the $A_{j}$ in $S_{m-1, n}^{\circ}(R)_{\text {alg }}$. We have

$$
g(\xi, \rho)=Q(\xi, \rho) \cdot W+\sum_{i=0}^{s-1} r_{i}\left(\xi^{\prime}, \rho\right) \xi_{m}^{i}
$$

with the $r_{i}\left(\xi^{\prime}, \rho\right)$ in $S_{m-1, n}^{\circ}(\widehat{R})$.

Assume first that $W$ is irreducible and separable and let $\bar{\xi}_{m, j}, j=1, \ldots, s$, be the zeros of $W$ in the algebraic closure of the quotient field of $S_{m, n}^{\circ}(\widehat{R})$. Since $g(\xi, \rho)$ is algebraic, so is $g\left(\xi^{\prime}, \bar{\xi}_{m, j}, \rho\right)$ for each $j$. Then for $j=1, \ldots, s$ we have

$$
g\left(\xi^{\prime}, \bar{\xi}_{m, j}, \rho\right)=\sum_{i=0}^{s-1} r_{i}\left(\xi^{\prime}, \rho\right) \bar{\xi}_{m, j}^{i} .
$$

Considering these as equations in the unknowns $r_{i}$, we see that the coefficient matrix is nonsingular, and hence the $r_{i}$ are algebraic.

In the nonirreducible case, we do Weierstrass Division successively by the irreducible factors of $W$, observing that they will also be regular in $\xi_{m}$.

Finally, we must consider the case that $W$ is irreducible, but not separable, i.e. $W$ is a polynomial in $\xi_{m}^{p}$, where $p$ is the characteristic of $R$, say $W\left(\xi^{\prime}, \xi_{m}, \rho\right)=W_{1}\left(\left(\xi^{\prime}, \xi_{m}^{p}, \rho\right)\right.$. We proceed by induction on the degree of $W$. First, $W_{1}$ has degree $s / p=s^{\prime}<s$ and is regular of degree $s^{\prime}$. Write

$$
g=\sum_{i=0}^{p-1} g_{i}\left(\xi^{\prime}, \xi_{m}^{p}, \rho\right) \xi_{m}^{i}
$$

Then $g_{i} \in S_{m, n}^{\circ}(R)_{\text {alg }}$ and hence by induction there are $r_{i, j} \in S_{m-1, n}^{\circ}(R)_{\text {alg }}$ such that

$$
g_{i}\left(\xi^{\prime}, \eta, \rho\right)=Q_{i}\left(\xi^{\prime}, \eta, \rho\right) W_{1}\left(\xi^{\prime}, \eta, \rho\right)+\sum_{j=0}^{s^{\prime}-1} r_{i, j}\left(\xi^{\prime}, \rho\right) \eta^{j} .
$$

Replacing $\eta$ by $\xi_{m}^{p}$ we see that

$$
\begin{aligned}
g\left(\xi^{\prime}, \xi_{m}, \rho\right) & =\sum_{i=0}^{p-1} g_{i}\left(\xi^{\prime}, \xi_{m}^{p}, \rho\right) \xi_{m}^{i} \\
& =\left(\sum_{i=0}^{p-1} Q_{i}\left(\xi^{\prime}, \xi_{m}^{p}, \rho\right) \xi_{m}^{i}\right) \cdot W+\sum_{i=0}^{p-1} \sum_{j=1}^{s^{\prime}-1} r_{i, j}\left(\xi^{\prime}, \rho\right) \xi_{m}^{p \cdot j+i} .
\end{aligned}
$$


From Theorem 4.6.5 and Remark 4.6.4 we have

Corollary 4.6.6. If $K$ is Henselian and $R \subset K^{\circ}$ is either a field or an excellent discrete valuation ring, then:

(i) $\left\{S_{m, n}^{\circ}(R)_{\mathrm{alg}}\right\}_{m, n}$ is a separated Weierstrass system.

(ii) The elements of $S_{m, n}^{\circ}(R)_{\mathrm{alg}}$ define analytic functions $\left(K^{\circ}\right)^{m} \times\left(K^{\circ \circ}\right)^{n} \rightarrow K^{\circ}$. Indeed, these functions are existentially definable uniformly in $K$. Hence $K$ has a natural analytic $\left\{S_{m, n}^{\circ}(R)_{\mathrm{alg}}\right\}_{m, n}$-structure.

(iii) Taking $R=\mathbb{Q}, \mathbb{Z}_{p}^{\mathrm{alg}}$ the algebraic $p$-adic integers, and $\mathbb{F}_{p}$ the p-element field, gives prime (i.e. smallest) analytic structures on all Henselian fields, depending only on the characteristic and residue characteristic of the field.

\section{Subdomains of $K_{\mathrm{alg}}^{\circ}$ and their rings of separated analytic functions}

In this section, we develop the basis of a theory of analytic functions on a $K$-annulus (an irreducible $K$-domain in $K_{\text {alg }}^{\circ}$ in the terminology of ["[LR1]), when $K$ carries a separated analytic $\mathcal{A}(K)$-structure. Here $\mathcal{A}=\left\{A_{m, n}\right\}$ is a (fixed) separated Weierstrass system and $K$ is a field with separated analytic $\mathcal{A}$-structure. We assume in this section that $\operatorname{ker}\left(\sigma_{0,0}\right)=(0)$ and hence that $K$ has analytic $\mathcal{A}(K)$-structure (cf. Definition 4.5.6. Theorem 4.5.7). Our main result is that any analytic function on any $K$-annulus equals a unit times a rational function (cf. Theorem 5.5.2). From this it follows that if $\tau$ is a term in the language of valued fields with analytic $\mathcal{A}(K)$-structure, then there is a cover of $K_{\text {alg }}^{\circ}$ by finitely many $K$-annuli on each of which $\tau$ is a rational function times a strong unit (cf. Theorem 5.5.3). This is what is needed for most model-theoretic applications. In Section 5.6 we prove some stronger results under stronger assumptions.

The results in this section extend some the results of [CLR1, Section 3], as well as some of those of [DHM]. They also extend the classical results of [FP Section 2.2] to the quasi-affinoid and the nonalgebraically closed cases. An alternative definition of analytic structures is given in [Scan2] where a statement similar to Theorem 5.5.3 is an axiom (more or less instead of our axiom (c) which seems to us more readily verifiable for the examples in Section 4.4.

We present a more complete theory than the minimum needed for the above-mentioned result about terms. The reader interested only in that application should consult Remark 5.5.5 for the shortest path to that result. The complications in the proofs are a consequence of the lack of completeness-completeness is a key ingredient of the proofs in the classical (affinoid) algebraically closed case. It is also a key ingredient in the development of affinoid algebra and geometry ([BGR]) and quasi-affinoid algebra and geometry ([LR1]). In Section 5.6 we assume a weak consequence of completeness (that holds in most of the standard examples), give somewhat easier proofs, and also establish a second "Mittag-Leffler" type theorem (Theorem 5.6.5.)

In this whole section, $\mathcal{A}$ is a separated Weierstrass system and any separated analytic $\mathcal{A}$-structure $\sigma$ is assumed to satisfy $\operatorname{ker}\left(\sigma_{0,0}\right)=(0)$. Hence, if $K$ has analytic $\mathcal{A}$-structure, then it has analytic $\mathcal{A}(K)$-structure (cf. Definition 4.5.6, Theorem 4.5.7). 
With some care, all results and definitions in this section and Section 6 can be adapted to the case of strictly convergent Weierstrass systems $(\pi \neq 1)$, where one uses the maximal algebraic unramified extension in place of $K_{\text {alg }}$ (see [CLR1]).

\subsection{Definitions and notation}

Definition 5.1.1 ( $K$-annulus). Let $K$ be a Henselian valued field.

(a) A $K$-annulus formula is a formula $\varphi$ of the form

$$
\left|p_{0}(x)\right| \square_{0} \varepsilon_{0} \wedge \bigwedge_{i=1}^{L} \varepsilon_{i} \square_{i}\left|p_{i}(x)\right|,
$$

where the $p_{i} \in K^{\circ}[x]$ are monic and irreducible, the $\varepsilon_{i}$ in $\sqrt{|K \backslash\{0\}|}$ and the $\square_{i}$ are in $\{<, \leq\}$. Define $\bar{\square}_{i}$ by $\left\{\square_{i}, \bar{\square}_{i}\right\}=\{<, \leq\}$. We require further that the sets

$$
\mathcal{H}_{i}:=\left\{x \in K_{\text {alg }}:\left|p_{i}(x)\right| \bar{\square}_{i} \varepsilon_{i}\right\}, \quad i=1, \ldots, L,
$$

be disjoint and contained in $\left\{x \in K_{\text {alg }}:\left|p_{0}(x)\right| \square_{0} \varepsilon_{0}\right\}$.

(b) The corresponding $K$-annulus is

$$
\mathcal{U}_{\varphi}:=\left\{x \in K_{\text {alg }}: \varphi(x)\right\}
$$

(If $K_{1} \supset K_{\text {alg }}$ is a field then $\varphi$ also defines a subset of $K_{1}$. We shall also refer to this as $\mathcal{U}_{\varphi}$ and also call it a $K$-annulus. No confusion will result.) The $K$-holes of $\varphi$, sometimes called the holes of $\mathcal{U}_{\varphi}$, are the sets $\mathcal{H}_{i}$. A $K$-annulus of the form $\left\{x \in K_{\text {alg }}:\left|p_{0}(x)\right| \square_{0} \varepsilon_{0}\right\}$ is called a $K$-disc or just a disc.

(c) A $K$-annulus formula $\varphi$ and the $K$-annulus $\mathcal{U}_{\varphi}$ are called linear if the $p_{i}$ are all linear and the $\varepsilon_{i}$ are in $|K| \backslash\{0\}$.

(d) A $K$-annulus fomula $\varphi$ and the $K$-annulus $\mathcal{U}_{\varphi}$ are called closed (resp. open) if all the $\square_{i}$ are $\leq$ (resp. $<$ ).

(e) A $K$-annulus formula is called good if the $p_{i}$ are of lowest possible degrees among all $K$-annulus formulas defining the same $K$-annulus.

Instead of requiring in (a) that the $p_{i}$ be irreducible and allowing the $\varepsilon_{i}$ in $\sqrt{|K \backslash\{0\}|}$, we could require that the $\varepsilon_{i}$ are in $|K \backslash\{0\}|$ and allow the $p_{i}$ to be powers of irreducible monic polynomials.

We shall often let $\varepsilon$ denote an element of $|K \backslash\{0\}|$ or an element of $K$ of that size, which will be clear from the context.

\section{Lemma 5.1.2. Let $K$ be Henselian.}

(i) Let $p \in K[x]$ be irreducible and let $\square \in\{<, \leq\}$. Then for every $\delta \in \sqrt{|K \backslash\{0\}|}$ there is an $\varepsilon \in \sqrt{|K \backslash\{0\}|}$ such that for every $x \in K_{\mathrm{alg}},|p(x)| \square \varepsilon$ if, and only if, for some zero $\alpha$ of $p,|x-\alpha| \square \delta$.

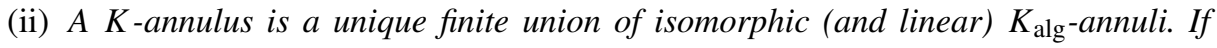
$K=K_{\mathrm{alg}}$ then all $K$-annulus formulas are linear. 
(iii) Any two $K$-discs (cf. Definition 5.1.1 b)) $\mathcal{U}_{1}$ and $\mathcal{U}_{2}$ are either disjoint or one is contained in the other.

(iv) For any two $K$-annuli $\mathcal{U}_{1}$ and $\mathcal{U}_{2}$, if $\mathcal{U}_{1} \cap \mathcal{U}_{2} \neq \emptyset$ then $\mathcal{U}_{1} \cap \mathcal{U}_{2}$ is a $K$-annulus.

(v) The complement of a $K$-annulus $\mathcal{U}$ (i.e. $K_{\mathrm{alg}}^{\circ} \backslash \mathcal{U}$ ) is a finite union of $K$-annuli.

(vi) Every set of the form

$$
\mathcal{U}=\left\{x \in K_{\text {alg }}^{\circ}:\left|p_{0}(x)\right| \square_{0} \varepsilon_{0} \wedge \bigwedge_{i=1}^{s} \varepsilon_{i} \square_{i}\left|p_{i}(x)\right|\right\}
$$

with the $p_{i}$ irreducible and the $\varepsilon_{i}$ in $\sqrt{|K \backslash\{0\}|}$ is described by a $K$-annulus formula.

(vii) Every $K$-annulus is described by a good $K$-annulus formula.

(viii) If for a finite collection of $K$-annulus formulas $\varphi_{i}$ the $K$-annuli $\mathcal{U}_{\varphi_{i}}$ cover $K_{\mathrm{alg}}^{\circ}$, then for any $K^{\prime} \supset K$ the corresponding $K^{\prime}$-annuli cover $K^{\prime \circ}$ (cf. Definition 5.1.1 b)).

Proof. Exercise (or see [CLR1, Lemma 3.2]).

Definition 5.1.3 (Rings of analytic functions). Let $K$ have separated analytic $\mathcal{A}$-structure, and let $\varphi$ be a $K$-annulus formula as in Definition 5.1.1 (a). Define the corresponding generalized rings of fractions over $K^{\circ}$, resp. $K$, by

$$
\mathcal{O}_{K}(\varphi):=A_{m+1, n}(K) /\left(p_{0}^{\ell_{0}}(x)-a_{0} z_{0}, p_{1}^{\ell_{1}}(x) z_{1}-a_{1}, \ldots, p_{L}^{\ell_{L}}(x) z_{L}-a_{L}\right)
$$

and

$$
\mathcal{O}_{K}^{\dagger}(\varphi):=K \otimes_{K^{\circ}} \mathcal{O}_{K}(\varphi),
$$

where $a_{i} \in K^{\circ},\left|a_{i}\right|=\varepsilon_{i}^{\ell_{i}}, m+n=L+1,\left\{z_{0}, \ldots, z_{L}\right\}$ is the set $\left\{\xi_{2}, \ldots, \xi_{m+1}\right.$, $\left.\rho_{1}, \ldots, \rho_{n}\right\}$ and $x$ is $\xi_{1}$ and $z_{i}$ is a $\xi$ or $\rho$ variable depending, respectively, on whether $\square_{i}$ is $\leq$ or $<$. By Weierstrass Division, each $f \in \mathcal{O}_{K}^{\dagger}(\varphi)$ defines a function $\mathcal{U}_{\varphi} \rightarrow K_{\mathrm{alg}}$ via the analytic structure on $K_{\text {alg }}$ given by Theorem 4.5.11. Denote this function by $f^{\sigma}$. Let $\mathcal{O}_{K}^{\sigma}(\varphi)$ be the image of $\mathcal{O}_{K}^{\dagger}(\varphi)$ under $f \mapsto f^{\sigma}$ and call $\mathcal{O}_{K}^{\sigma}(\varphi)$ the ring of analytic functions on $\mathcal{U}_{K}(\varphi)$.

Clearly, $f^{\sigma}$ maps $\mathcal{U}_{\varphi} \cap K^{\prime}$ into $K^{\prime}$ for any field $K \subset K^{\prime} \subset K_{\text {alg }}$ (cf. Theorem 4.5.11).

The mapping $f \mapsto f^{\sigma}$ is obviously a surjective $K$-algebra homomorphism. We do not know if it is always injective, though we do prove it is for some annuli of particularly simple forms (Lemmas 5.3.6 and 5.3.7 and Corollary 5.3.10. We show in Section 5.6 (Theorem 5.6.3 that under the stronger assumption that $K$ has strong separated analytic structure, this homomorphism is injective for all $K$-annuli.

Definition 5.1.4 (Units). Let $f^{\sigma}$ be a unit in $\mathcal{O}_{K}^{\sigma}(\varphi)$. Suppose that there is some $\ell \in \mathbb{N}$ and $c \in K$ such that $\left|\left(f^{\sigma}(x)\right)^{\ell}\right|=|c|$ for all $x \in \mathcal{U}_{\varphi}$. Suppose also that there exists a nonzero polynomial $\underset{\sim}{P}(\xi) \in \widetilde{K}[\xi]$ such that $P\left(\left(c^{-1}\left(f^{\sigma}(x)\right)^{\ell}\right)^{\sim}\right)=0$ for all $x \in \mathcal{U}_{\varphi}$, where $^{\sim}: K_{\text {alg }}^{\circ} \rightarrow \widetilde{K}_{\text {alg }}$ is the natural projection to the residue field. Then we call $f$ a strong unit. We call $f$ a very strong unit if moreover $|f(x)|=1$ and $(f(x))^{\sim}=1$ for all $x \in \mathcal{U}_{\varphi}$. We call $f \in \mathcal{O}_{K}^{\dagger}(\varphi)$ a (very) strong unit if $f^{\sigma}$ is. 
Examples 5.1.5. (i) If $f \in \mathcal{O}_{K}(\varphi), f=1+g$ with

$$
g \in\left(A_{m+1, n}(K)\right)^{\circ} /\left(p_{0}^{\ell_{0}}(x)-a_{0} z_{0}, p_{1}^{\ell_{1}}(x) z_{1}-a_{1}, \ldots, p_{L}^{\ell_{L}}(x) z_{L}-a_{L}\right),
$$

then $f$ is a unit by Remark 4.1 .7 and since $K$ has analytic $\mathcal{A}(K)$-structure. Hence, in this case $f$ is a very strong unit.

(ii) Let $\varphi$ be the $K$-annulus formula $|x|<1$, and $\mathcal{U}_{\varphi}=K_{\text {alg }}^{\circ \circ}$ be the corresponding open disc. Then $\mathcal{O}_{K}(\varphi)=A_{0,1}$, as expected. Namely, by definition, $\mathcal{O}_{K}(\varphi)=$ $A_{1,1}(K) /\left(x-\rho_{1}\right)$ with $x$ a $\xi$-variable. But since $x-\rho_{1}$ is regular in $x$ of degree 1 (cf. Definition 4.1.1 (i)), for any $g$ in $A_{1,1}$ there exist unique $q \in A_{1,1}$ and $r \in A_{0,1}$ such that $g=q\left(x-\rho_{1}\right)+r$. This shows that there is a well defined surjective map from $\mathcal{O}_{K}(\varphi)$ to $A_{0,1}$, namely sending the class of $g$ to the class of $r$, with inverse just the projection from $A_{0,1}$ to $\mathcal{O}_{K}(\varphi)$.

(iii) Let $\varphi$ be the $K$-annulus formula $|x| \leq 1$, and $\mathcal{U}_{\varphi}=K_{\text {alg }}^{\circ}$ be the corresponding closed disc. Then clearly $\mathcal{O}_{K}(\varphi)=A_{1,0}$.

(iv) An example of a strong unit which is not a constant plus a small function is as follows. Let $\widetilde{K}=\mathbb{R}$. The annulus formula $\left|x^{2}+1\right|<1$ gives a $K$-annulus $\mathcal{U}$ on which the identity function $x \mapsto x$ is a strong unit. The $K$-disc $\mathcal{U}$ consists of two open $K_{\text {alg }}$-discs centered at $i$ and $-i$. For details, see [CLR1].

\section{Notation}

With the notation from Definition 5.1.3 in particular, $\rho=\left(\rho_{1}, \ldots, \rho_{n}\right)$, we define

$$
\mathcal{O}_{K}(\varphi)^{\circ}:=\left(K^{\circ \circ}, \rho\right) \mathcal{O}_{K}(\varphi), \quad \mathcal{O}_{K}^{\dagger}(\varphi)^{\circ}:=\mathcal{O}_{K}(\varphi), \quad \mathcal{O}_{K}^{\dagger}(\varphi)^{\circ \circ}:=\mathcal{O}_{K}(\varphi)^{\circ} .
$$

We will use the suggestive notation

$$
f=\sum_{\nu} a_{v}(x)\left(\frac{p_{0}(x)^{\ell_{0}}}{a_{0}}\right)^{\nu_{0}}\left(\frac{a_{1}}{p_{1}(x)^{\ell_{1}}}\right)^{\nu_{1}} \cdots\left(\frac{a_{n}}{p_{n}(x)^{\ell_{n}}}\right)^{v_{n}}
$$

to denote the image of $f=\sum_{\nu} a_{\nu}\left(\xi_{1}\right) z_{0}^{\nu_{0}} z_{0}^{\nu_{0}} z_{1}^{\nu_{1}} \cdots z_{n}^{\nu_{n}} \in A_{m+1, n}$ in $\mathcal{O}_{K}^{\dagger}(\varphi)$ or $\mathcal{O}_{K}^{\sigma}(\varphi)$.

We need to study the rings of functions on annuli of some particularly simple forms.

Definition 5.1.6. (i) A linear $K$-annulus of the form

$$
\left\{x \in K_{\mathrm{alg}}:\left|x-a_{0}\right| \leq \varepsilon \text { and } \bigwedge_{i=0}^{n}\left|x-a_{i}\right| \geq \varepsilon\right\}
$$

for some $\varepsilon \in \sqrt{|K \backslash\{0\}|}, \varepsilon \leq 1$, and $a_{i} \in K^{\circ}$ is called thin. A general $K$ annulus $\mathcal{U}$ is called thin if each of the linear $K_{\text {alg-annuli }} \mathcal{U}_{i}$ corresponding to $\mathcal{U}$ as in Lemma 5.1.2 $\mathrm{ii}$ ) is a thin linear $K_{\mathrm{alg}}$-annulus. 
(ii) A $K$-annulus $\mathcal{U}$ of the form

$$
\left\{x \in K_{\mathrm{alg}}: \varepsilon_{1}<|p(x)|<\varepsilon_{0}\right\},
$$

where $p \in K[x]$ is irreducible, is called a Laurent annulus. We call a Laurent $K$-annulus $\mathcal{U}$ simple if each of the linear $K_{\text {alg }}$-annuli $\mathcal{U}_{i}$ corresponding to $\mathcal{U}$ as in

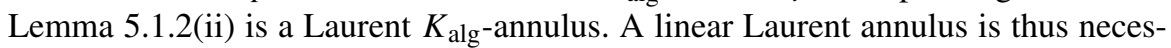
sarily simple.

(iii) If the linear $K$-annulus

$$
\left\{x \in K_{\text {alg }}:\left|x-a_{0}\right| \leq \varepsilon \text { and } \bigwedge_{i=0}^{n}\left|x-a_{i}\right| \geq \varepsilon\right\}
$$

is thin and $\varepsilon^{\prime}<\varepsilon<\varepsilon^{\prime \prime}$, and $\mathcal{U}^{\prime}, \mathcal{U}^{\prime \prime}$ are defined by

$$
\begin{aligned}
\mathcal{U}^{\prime} & :=\left\{x \in K_{\mathrm{alg}}:\left|x-a_{0}\right| \leq \varepsilon \text { and }\left|x-a_{0}\right| \geq \varepsilon^{\prime} \text { and } \bigwedge_{i=1}^{n}\left|x-a_{i}\right| \geq \varepsilon\right\}, \\
\mathcal{U}^{\prime \prime} & :=\left\{x \in K_{\mathrm{alg}}:\left|x-a_{0}\right| \leq \varepsilon^{\prime \prime} \text { and } \bigwedge_{i=0}^{n}\left|x-a_{i}\right| \geq \varepsilon\right\}
\end{aligned}
$$

we call $\mathcal{U}^{\prime}, \mathcal{U}^{\prime \prime}$ almost thin. A general $K$-annulus $\mathcal{U}$ is called almost thin if each of the linear $K_{\text {alg-annuli }} \mathcal{U}_{i}$ corresponding to $\mathcal{U}$ as in Lemma 5.1.2 ii) is an almost thin

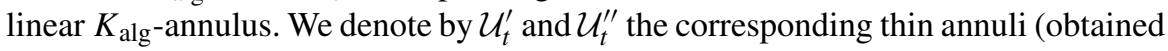
by replacing $\varepsilon^{\prime}$ and $\varepsilon^{\prime \prime}$ by $\varepsilon$ in the above definitions).

(iv) We call two $K$-annuli adjacent if their union is a $K$-annulus. We say that two $K$ annuli overlap if their intersection is nonempty.

(v) We call a finite cover $\left\{\mathcal{U}_{i}\right\}$ of $\mathcal{U}$ by annuli $\mathcal{U}_{i}$ rigid if for each $i, j$ there is a finite sequence $\mathcal{U}_{i}=\mathcal{U}_{i_{1}}, \ldots, \mathcal{U}_{i_{m}}=\mathcal{U}_{j}$ from the cover such that for each $\ell=1, \ldots, m-1$, $\mathcal{U}_{i_{\ell}}$ and $\mathcal{U}_{i_{\ell+1}}$ overlap.

Remark 5.1.7. (i) An open $K$-annulus with only one $K$-hole is Laurent, by Lemma 5.1.2(i).

(ii) An almost thin annulus is a thin annulus $(\mathcal{U})$ that has been "thickened" either in its hole around $a_{0}\left(\mathcal{U}^{\prime}\right)$ or in its "hole at $\infty$ " $\left(\mathcal{U}^{\prime \prime}\right)$.

The two types of almost thin annuli are equivalent for many purposes. The proof of the following lemma is a simple calculation.

Lemma 5.1.8. The two types of almost thin linear annuli $\left(\mathcal{U}^{\prime}, \mathcal{U}^{\prime \prime}\right)$ in the definition, and their rings of functions $\left(\mathcal{O}\left(\mathcal{U}^{\prime}\right), \mathcal{O}\left(\mathcal{U}^{\prime \prime}\right)\right)$, are related by the change of variables

$$
y=\left(x-a_{1}\right)^{-1} .
$$


Lemma 5.1.9. Let $R(x) \in K(x)$ and $\varepsilon \in \sqrt{|K| \backslash\{0\}}$, let $\square \in\{<, \leq\}$, and let

$$
\mathcal{U}:=\left\{x \in K_{\text {alg }}^{\circ}:|R(x)| \square \varepsilon\right\} .
$$

There are finitely many $K$-annuli $\mathcal{U}_{i}, i=1, \ldots, L$, and a finite set $S \subset K_{\mathrm{alg}}^{\circ}$, such that $\mathcal{U} \backslash S=\left(\bigcup_{i=1}^{L} \mathcal{U}_{i}\right) \backslash S$ and for each $i$ there is an $f_{i} \in \mathcal{O}_{K}^{\dagger}\left(\mathcal{U}_{i}\right)$ such that

$$
\left.R(x)\right|_{\mathcal{U}_{i} \backslash S}=\left.f_{i}\right|_{\mathcal{U}_{i} \backslash S} .
$$

Proof. We may assume that $R(x)=x^{n_{0}} \prod_{i=1}^{s} p_{i}(x)^{n_{i}}$, where the $p_{i} \in K^{\circ}[x]$ are monic, irreducible and mutually prime and the $n_{i}$ are integers. The lemma is proved in [CLR1, Lemma 3.16].

\subsection{Results from [BGR]}

In this subsection we summarize some of the results of [BGR] that apply without change in our context. Only fairly special cases of these results are actually used (for example in the proof of Proposition 5.3.2.

Observe that a $K$-annulus is not always a $K_{\text {alg }}$-annulus. However, by Lemma 5.1.2(ii), a $K$-annulus is the disjoint union of a finite set of $K_{\text {alg }}$-annuli (and the corresponding rings of functions are related as in Proposition 5.3.11. These linear $K_{\mathrm{alg}}$-annuli are isomorphic via automorphisms of $K_{\text {alg }}$ over $K$. We are thus led to consider more general domains.

The following is used in Definition 5.2.2(iii) and afterwards in Section 5.5 .

Definition 5.2.1 (Residue norm). With the notation from Definition 5.1.3 for $f \in$ $\mathcal{O}_{K}(\varphi)$,

$$
\|f\| \leq 1
$$

means that there exists $g \in A_{m+1, n}(K)$ whose class in $\mathcal{O}_{K}(\varphi)$ is $f$ and such that $\|g\| \leq 1$, with $\|\cdot\|$ the Gauss norm (see just above Remark 4.1.10). This usage extends naturally to the case (Definition 5.2.2 below) that $\varphi$ is a domain formula.

Definition 5.2.2. We define the concepts of a domain formula $\varphi$, the corresponding domain $\mathcal{U}_{\varphi}$, generalized ring of $K^{\circ}$-fractions $\mathcal{O}_{K}(\varphi)$, generalized ring of $K$-fractions $\mathcal{O}_{K}^{\dagger}(\varphi)$ and ring of analytic functions $\mathcal{O}_{K}^{\sigma}(\mathcal{U})$ inductively as follows.

(i) The formula $\varphi:=\bigwedge_{i=1}^{m}\left(\left|\xi_{i}\right| \leq 1\right) \wedge \bigwedge_{i=1}^{n}\left(\left|\rho_{i}\right|<1\right)$ is a domain formula. The corresponding domain is $\left(K_{\mathrm{alg}}^{\circ}\right)^{m} \times\left(K_{\mathrm{alg}}^{\circ}\right)^{n}$, its generalized ring of $K^{\circ}$-fractions is $A_{m, n}(K)$, and its generalized ring of $K$-fractions is $A_{m, n}^{\dagger}(K):=K \otimes_{K^{\circ}} A_{m, n}(K)$.

(ii) If $\varphi, \mathcal{U}_{\varphi}, \mathcal{O}_{K}(\varphi)$ and $\mathcal{O}_{K}^{\dagger}(\varphi)$ are a domain formula and the corresponding domain and generalized ring of fractions, and $f_{1}, \ldots, f_{M}, g_{1}, \ldots, g_{N}, h \in \mathcal{O}_{K}^{\dagger}(\varphi)$ generate the unit ideal, then

$$
\varphi^{\prime}:=\varphi \wedge \bigwedge_{i=1}^{M}\left(\left|f_{i}\right| \leq|h|\right) \wedge \bigwedge_{i=1}^{N}\left(\left|g_{i}\right|<|h|\right)
$$


is a domain formula. The corresponding domain is

$$
\left\{x \in \mathcal{U}_{\varphi}: \bigwedge_{i=1}^{M}\left(\left|f_{i}(x)\right| \leq|h(x)|\right) \text { and } \bigwedge_{i=1}^{N}\left(\left|g_{i}(x)\right|<|h(x)|\right)\right\}
$$

and if $\mathcal{O}_{K}(\varphi)=A_{m, n}(K) / I$ then

$$
\mathcal{O}_{K}\left(\varphi^{\prime}\right)=A_{m+M, n+N}(K) / I^{\prime}
$$

where

$$
I^{\prime}=\left(I, f_{1}-\eta_{1} h, \ldots, f_{M}-\eta_{M} h, g_{1}-\tau_{1} h, \ldots, g_{N}-\tau_{N} h\right)
$$

(iii) We define the corresponding generalized ring of ( $K$-valued) fractions $\mathcal{O}_{K}^{\dagger}(\varphi)$ on $\mathcal{U}_{\varphi}$ by

$$
\mathcal{O}_{K}^{\dagger}(\varphi):=K \otimes_{K} \circ \mathcal{O}_{K}(\varphi)
$$

This is consistent with the notation $A_{m, n}^{\dagger}(K)=K \otimes_{K^{\circ}} A_{m, n}(K)$ from (i). We define

$$
\mathcal{O}_{K}^{\dagger}(\varphi)^{\circ}:=\left\{f \in \mathcal{O}_{K}(\varphi):\|f\| \leq 1\right\}, \quad \mathcal{O}_{K}^{\dagger}(\varphi)^{\circ \circ}:=\left(K^{\circ \circ}, \rho\right) \mathcal{O}_{K}^{\dagger}(\varphi)^{\circ},
$$

where $\mathcal{O}_{K}(\varphi)=A_{m, n}(K) / I$ and $\rho=\left(\rho_{1}, \ldots, \rho_{n}\right)$.

(iv) The corresponding ring of functions $\mathcal{O}_{K}^{\sigma}(\varphi)$ is the image of $\mathcal{O}_{K}^{\dagger}(\varphi)$ in the ring of functions $\mathcal{U}_{\varphi} \rightarrow K_{\text {alg }}$ under the mapping provided by the analytic $\mathcal{A}\left(K_{\text {alg }}\right)$-structure on $K_{\text {alg. }}$.

(v) We call a domain closed if all its defining inequalities are weak (i.e. $\leq$ ), and we call a domain open if all its defining inequalities are strict (i.e. $<$ ). In the case that $\mathcal{U}$ is closed its ring of $K^{\circ}$-fractions is of the form $A_{m, 0} / I$ and in the case it is open its ring of $K$-fractions is of the form $A_{0, n} / I$.

The following definition generalizes the notion of domains, and will only be used in the proofs later on in Section 5.5. No confusion should result with the notation of Definition 5.2.2.

Definition 5.2.3. If $J$ is an ideal in $A_{m, n}^{\dagger}(K)$, define

$$
\begin{aligned}
V(J) & :=\left\{(a, b) \in\left(K_{\text {alg }}^{\circ}\right)^{m} \times\left(K_{\text {alg }}^{\circ \circ}\right)^{n}: f^{\sigma}(a, b)=0 \text { for all } f \in J\right\}, \\
\mathcal{O}_{K}^{\dagger}(J) & :=A_{m, n}^{\dagger}(K) / J .
\end{aligned}
$$

The elements of $\mathcal{O}^{\dagger}(J)$ define functions on $V(J) \rightarrow K_{\text {alg via }} \sigma$ in a natural way, and we denote this ring of functions by $\mathcal{O}_{K}^{\sigma}(J)$. 
Remark 5.2.4. (i) If $\varphi$ is a $K$-annulus formula and $F$ is an algebraic extension of $K$, then $\varphi$ is a $F$-domain formula, even though $\varphi$ may not be an $F$-annulus formula.

(ii) Many of the results for domains, of [BGR] in the affinoid case, and of [LR1] in the quasi-affinoid case, hold also in the more general setting of Henselian fields with analytic structure, often with (slightly) modified proofs. To check exactly how proofs must be modified would be a cumbersome task. For example Proposition 5.3.11(ii) below would follow from the analogue of [BGR, Lemma 7.2.2.8] in the affinoid case. But the proof of that lemma in $[\mathrm{BGR}]$ uses much of that book. We will give a self-contained treatment, though we will quote some theorems from [BGR] when the proofs apply without modification.

(iii) From the Strong Noetherian Property we see that $A_{m, n}^{\dagger}(K)^{\circ}=A_{m, n}(K)$ and $A_{m, n}^{\dagger}(K)^{\circ \circ}=A_{m, n}(K)^{\circ}$. It is easy to see that for $f \in A_{m, n}^{\dagger}(K)$ we have $f \in A_{m, n}^{\dagger}(K)^{\circ}$ if and only if $\left|f^{\sigma}(a, b)\right| \leq 1$ for all $(a, b) \in\left(K_{\mathrm{alg}}^{\circ}\right)^{m} \times\left(K_{\mathrm{alg}}^{\circ}\right)^{n}$, which is the case if and only if $\|f\| \leq 1$, and that $f \in A_{m, n}^{\dagger}(K)^{\circ \circ}$ if and only if $\left|f^{\sigma}(a, b)\right|<1$ for all $(a, b) \in\left(K_{\mathrm{alg}}^{\circ}\right)^{m} \times\left(K_{\mathrm{alg}}^{\circ \circ}\right)^{n}$, which is the case if and only if $f \in\left(K^{\circ \circ}, \rho\right) A_{m, n}(K)^{\circ}$.

From Theorem 4.5.7 we know that the rings $A_{m, 0}(K)$ and $A_{0, n}(K)$ have Weierstrass Preparation, are closed under Weierstrass changes of variables, and (from the Strong Noetherian Property) have the property that for any $0 \neq f \in A_{m, 0}(K)\left(\right.$ or $\left.A_{0, n}(K)\right)$, there is an $a \in K$ such that $a f \in A_{m, 0}(K)$ (or $A_{0, n}(K)$ ) satisfies $\|a f\|=1$ and is preregular (and hence after a Weierstrass change of variables, regular) of some degree.

Proposition 5.2.5. The Rückert Theory of [BGR Sections 5.2.5 and 5.2.6] applies to the rings $A_{m, 0}^{\dagger}(K)$ and to the rings $A_{0, n}^{\dagger}(K)$. Hence these rings are Noetherian, factorial and normal (integrally closed in their fields of fractions).

Proof. The proofs of [BGR] apply.

From the Strong Noetherian Property and Weierstrass Preparation we have (cf. [BGR, Corollary 6.1.2.2])

Proposition 5.2.6 (Normalization).

(i) Let $J$ be an ideal in $A_{m, 0}^{\dagger}$. There is a nonnegative integer $d$ and a finite monomorphism

$$
A_{d, 0}^{\dagger}(K) \hookrightarrow A_{m, 0}^{\dagger}(K) / J
$$

(ii) Let $J$ be an ideal in $A_{0, n}^{\dagger}$. There is a nonnegative integer $d$ and a finite monomorphism

$$
A_{0, d}^{\dagger}(K) \hookrightarrow A_{0, n}^{\dagger}(K) / J
$$

As in [BGR, Section 7.1.2] we obtain

Proposition 5.2.7. The rings $A_{m, 0}^{\dagger}(K)$ and $A_{0, n}^{\dagger}(K)$ satisfy the Nullstellensatz. There is a one-to-one correspondence between the maximal ideals of $A_{m, 0}^{\dagger}$ (resp. $A_{0, n}^{\dagger}$ ) and the orbits of $\left(K_{\mathrm{alg}}^{\circ}\right)^{m}$ (resp. $\left.\left(K_{\mathrm{alg}}^{\circ \circ}\right)^{n}\right)$ under the Galois group of $K_{\mathrm{alg}}$ over $K$. 
Remark 5.2.8 (Norms). Let $J$ be an ideal in $A_{m, n}^{\dagger}(K)$. Then the elements of $A_{m, n}^{\dagger} / J$ define functions on $V(J)=\left\{x \in\left(K_{\mathrm{alg}}^{\circ}\right)^{m} \times\left(K_{\mathrm{alg}}^{\circ}\right)^{n}: f(x)=0\right.$ for all $\left.f \in J\right\}$. Since the proof of [LL2] or [LR2] (see also [LR3, Theorem 5.2]) shows that $K_{\text {alg }}$ admits quantifier elimination in the language with function symbols for the elements of $\mathcal{S}=\bigcup_{m, n} A_{m, n}^{\dagger}$, we see that the supremum seminorm $\|\cdot\|_{\text {sup }}$ on $V(J)$ is well defined and takes values in $\left|K_{\text {alg }}\right|$ (where $\|f\|_{\text {sup }}$ for $f \in A_{m, n}^{\dagger} / J$ is defined as the supremum of $|f(x)|$ over all $x$ in $V(J))$. If $A_{m, n}^{\dagger} / J$ is reduced then $\|\cdot\|_{\text {sup }}$ is a norm. The residue norm $\|\cdot\|_{J}$ on $A_{m, n}^{\dagger} / J$ is well defined in the standard examples (where $\|f\|_{J}$ is defined as the infimum of the Gauss norms of all representatives of $f$ in $A_{m, n}^{\dagger}$ ). We do not know if our assumptions on $\mathcal{S}$ are sufficient to guarantee that residue norms are always defined and, if $J$ is reduced, equivalent to the supremum norm. However, the notation of Definition 5.2.1 always makes sense, even if the residue norm is not defined. Clearly, for $f \in A_{m, n}^{\dagger}$, the supremum norm of $f$ equals the Gauss norm of $f$.

Remark 5.2.9. Normalization fails in general for ideals $J \subset A_{m, n}^{\dagger}(K)$ when $m, n>0$ (cf. [LR1, Example 2.3.5]). It is likely that the standard properties (the Nullstellensatz, unique factorization, etc.) could be established for these rings by adapting the proofs of [LR1]. This could be quite nontrivial to carry out and is not needed for the results of this paper.

Definition 5.2.10. With the notation from Definition 5.2.3, we shall call $f \in \mathcal{O}^{\dagger}(J)$ $\left(=A_{m, n}^{\dagger} / J\right)$ power bounded if $\left|f^{\sigma}(x)\right| \leq 1$ for all $x \in V(J)$, and topologically nilpotent if $\left|f^{\sigma}(x)\right|<1$ for all $x \in V(J)$. We shall say that $f \in \mathcal{O}^{\dagger}(V(J))$ is strongly power bounded if

$$
f^{n}=A_{1} f^{n-1}+A_{2} f^{n-2}+\cdots+A_{n}
$$

where for each $i$ there is an $a_{i} \in A_{m, n}(K)$ such that $a_{i} \in A_{i}+J$ with $A_{i}$ in $A_{m, n}^{\dagger}(K)$. We say that $f$ is strongly topologically nilpotent if the $a_{i}$ are in $A_{m, n}(K)^{\circ}=\left(K^{\circ \circ}, \rho\right) A_{m, n}(K)$. We call $f^{\sigma}$ (strongly) power bounded or (strongly) topologically nilpotent if $f$ is.

Remark 5.2.11. Observe that $f \in A_{m, n}^{\dagger}(K)$ is power bounded if and only if $f \in A_{m, n}(K)$, and $f$ is topologically nilpotent if and only if $f \in A_{m, n}(K)^{\circ}$. Since we allow $K$ to have rank $>1$, it is possible that there are $\alpha, \beta \in K$ with $|\alpha|>1$ and $\left|\alpha^{n}\right|<|\beta|$ for all $n \in \mathbb{N}$. By our definition $\alpha$ is not power bounded.

We have

Proposition 5.2.12 ([BGR, Proposition 3.8.1.7]). Let $\Phi: B \rightarrow A$ be an integral torsion free $K$-algebra monomorphism between two $K$-algebras $A$ and $B$, where $B$ is an integrally closed integral domain.

(a) $|f|_{\text {sup }}=\max _{1 \leq i \leq n}\left|b_{i}\right|_{\text {sup }}^{1 / i}$ for $f \in A$ where

$$
f^{n}+\Phi\left(b_{1}\right) f^{n-1}+\cdots+\Phi\left(b_{n}\right)=0
$$

is the (unique) integral equation of minimal degree for $f$ over $\Phi(B)$. 
(b) For $B=A_{m, 0}^{\dagger}$ or $B=A_{0, n}^{\dagger}$, if $f$ is power bounded, then it is strongly power bounded, and if $f$ is topologically nilpotent, then it is strongly topologically nilpotent.

Proposition 5.2.13. Let $\varphi$ be a domain formula, let $f_{1}, \ldots, f_{M}, g_{1}, \ldots, g_{N}, h \in \mathcal{O}_{K}^{\dagger}(\varphi)$ and suppose that $h$ is a unit in $\mathcal{O}_{K}^{\dagger}(\varphi)$, each $f_{i} / h$ is strongly power bounded and each $g_{i} / h$ is strongly topologically nilpotent. Let $\psi=\varphi \wedge \bigwedge_{i=1}^{M}\left|f_{i}\right| \leq|h| \wedge \wedge_{j=1}^{N}\left|g_{j}\right|<|h|$. Then $\mathcal{O}_{K}^{\dagger}(\psi)=\mathcal{O}_{K}^{\dagger}(\varphi)$.

Proof. [BGR, Proposition 6.1.4.3] or [LR1, Proposition 5.3.2].

From Propositions 5.2.5, 5.2.6, 5.2.12 and 5.2.13 we have the following corollary. In Corollary 5.2.14 we prove a similar result for all $K$-annuli.

Corollary 5.2.14. If $\varphi$ is an open or a closed domain formula then $\mathcal{O}_{K}(\varphi)=\mathcal{O}_{K}\left(\mathcal{U}_{\varphi}\right)$ i.e. $\mathcal{O}_{K}(\varphi)$ depends only on the domain $\mathcal{U}_{\varphi}$ and not on the particular description $\varphi$.

Remark 5.2.15. In case 2 of the proof of Proposition 5.3.2 we shall use annuli or discs of radius $>1$ and their associated rings. To give an example of such a disc, if $a \in K$, $|a|>1$, the annulus formula $\varphi:=(|x|<|a|) \wedge\left(\varepsilon_{1}<\left|p_{1}(x)\right|\right)$ defines the annulus $\mathcal{U}_{\varphi}=\left\{x \in K_{\text {alg }}: \varphi(x)\right\}$ and generalized ring of fractions

$$
\mathcal{O}_{K}^{\dagger}(\varphi):=\left\{f\left(\frac{\rho_{1}}{a}, \frac{a_{1}}{p_{1}^{\ell_{1}}\left(\rho_{1}\right)}\right): f \in A_{0,2}\right\} .
$$

Here $\left|a_{1}\right|=\varepsilon_{1}^{\ell_{1}} \in|K|$. It is clear that

$$
\mathcal{O}_{K}^{\dagger}(\varphi) \hookrightarrow \mathcal{O}_{K}^{\dagger}\left(\varepsilon_{1}<\left|p_{1}(x)\right|\right)=\mathcal{O}_{K}^{\dagger}\left(|x| \leq 1 \wedge \varepsilon_{1}<\left|p_{1}(x)\right|\right)
$$

by $\rho_{1} \mapsto \xi_{1}$. In this example $\varphi$ is an open annulus formula, $\mathcal{U}_{\varphi}$ is open and Propositions 5.2.5, 5.2.6 5.2.7, 5.2.12 and Corollary 5.2.14 apply to the rings $\mathcal{O}_{K}^{\dagger}(\varphi)$ and $\mathcal{O}_{K}^{\sigma}(\varphi)$.

\subsection{General K-annuli, part 1}

In this subsection we prove some preliminary results about $K$-annuli.

Proposition 5.3.1. Let

$$
\varphi:=\left|p_{0}(x)\right| \square_{0} \varepsilon_{0} \wedge \bigwedge_{i=1}^{L} \varepsilon_{i} \square_{i}\left|p_{i}(x)\right|
$$

be a good annulus formula and suppose that $P(x) \in K^{\circ}[x]$ is monic and irreducible and that $\alpha \in \mathcal{U}_{K}(\varphi)$ is a zero of $P(x)$. Let $f \in \mathcal{O}_{K}^{\dagger}(\varphi)$ and suppose that $f^{\sigma}(\alpha)=0$. Then there is a $g \in \mathcal{O}_{K}^{\dagger}(\varphi)$ such that $f(x)=P(x) \cdot g(x)$, i.e. $P(x)$ divides $f(x)$ in $\mathcal{O}_{K}^{\dagger}(\varphi)$. 
Proof. We may suppose that

$$
f=\sum_{\nu} b_{\nu}(x)\left(\frac{p_{0}(x)^{\ell_{0}}}{a_{0}}\right)^{\nu_{0}}\left(\frac{a_{1}}{p_{1}(x)^{\ell_{1}}}\right)^{\nu_{1}} \cdots\left(\frac{a_{n}}{p_{n}(x)^{\ell_{n}}}\right)^{v_{n}}
$$

where $\operatorname{deg} b_{v}(x)<\ell_{0} \operatorname{deg} p_{0}$. Let $Q_{0} \in K^{\circ}[x]$ be the minimal polynomial of $p_{0}^{\ell_{0}}(\alpha) / a_{0}$, and let $Q_{i}$ be the minimal polynomial of $a_{i} / p_{i}^{\ell_{i}}(\alpha)$ for $i=1, \ldots, n$. Then each $Q_{i}$ is regular in the appropriate sense for a variable corresponding to the inequality $\square_{i}$, and by Weierstrass Division

$$
f(x)=R(x)+g_{0} \cdot Q_{0}\left(\frac{p_{0}(x)^{\ell_{0}}}{a_{0}}\right)+\sum_{i=1}^{n} g_{i} \cdot Q_{i}\left(\frac{a_{i}}{p_{i}(x)^{\ell_{i}}}\right)
$$

where $R(x)$ is a rational function in $K(x)$ whose denominator is a product of powers of the $p_{i}(x), i=1, \ldots, n$. Let $R(x) \equiv r(x) \bmod P(x)$ where $r(x) \in K[x]$ has degree $<\operatorname{deg} P$ and note that

$$
Q_{0}\left(\frac{p_{0}(x)^{\ell_{0}}}{a_{0}}\right) \equiv 0 \bmod P(x)
$$

and, for $i=1, \ldots, n$, that

$$
Q_{i}\left(\frac{a_{i}}{p_{i}(x)^{\ell_{i}}}\right) \equiv 0 \bmod P(x)
$$

in $\mathcal{O}_{K}^{\dagger}(\varphi)$. Thus we have written

$$
f(x)=r(x)+g(x) \cdot P(x)
$$

where $g(x) \in \mathcal{O}_{K}^{\dagger}(\varphi)$ and $\operatorname{deg} r<\operatorname{deg} P$. Then $r(\alpha)=0$ and hence $r(x) \equiv 0$.

Proposition 5.3.2. If $\varphi$ and $\psi$ are $K$-annulus formulas and $\mathcal{U}_{\varphi} \subseteq \mathcal{U}_{\psi}$ then $\mathcal{O}_{K}^{\dagger}(\psi) \subseteq$ $\mathcal{O}_{K}^{\dagger}(\varphi)$

Proof. Let

$$
\varphi=p_{0}(x) \square_{0} \varepsilon_{0} \wedge \bigwedge_{i=1}^{n} \varepsilon_{i} \square_{i} p_{i}(x), \quad \psi=p_{0}^{\prime}(x) \square_{0}^{\prime} \varepsilon_{0}^{\prime} \wedge \bigwedge_{i=1}^{n^{\prime}} \varepsilon_{i}^{\prime} \square_{i}^{\prime} p_{i}^{\prime}(x) .
$$

By Corollary 5.2.13 it is enough to show: if $\square_{0}^{\prime}$ is $\leq$ then $p_{0}^{\prime \ell_{0}^{\prime}} / a_{0}^{\prime}$ is strongly power bounded in $\mathcal{O}_{K}^{\dagger}(\varphi)$; if $\square_{0}^{\prime}$ is $<$ then $p_{0}^{\prime \ell_{0}^{\prime}} / a_{0}^{\prime}$ is strongly topologically nilpotent in $\mathcal{O}_{K}^{\dagger}(\varphi)$; if $\square_{i}^{\prime}$ is $\leq$ then $a_{i}^{\prime} / p_{i}^{\prime \ell_{i}^{\prime}}$ is strongly power bounded in $\mathcal{O}_{K}^{\dagger}(\varphi)$; and if $\square_{i}^{\prime}$ is $<$ then $a_{i}^{\prime} / p_{i}^{\prime \ell_{i}^{\prime}}$ is strongly topologically nilpotent in $\mathcal{O}_{K}^{\dagger}(\varphi)$. We will check some of these cases and leave the rest to the reader.

Case 1. $\square_{0}$ is $\leq$. Consider $\varphi_{0}:=\left|p_{0}(x)\right| \leq \varepsilon_{0}$. Then $\mathcal{O}_{K}^{\dagger}\left(\varphi_{0}\right)=A_{2,0}^{\dagger} /\left(p_{0}^{\ell_{0}}\left(\xi_{1}\right)-\right.$ $\left.a_{0} \eta_{1}\right)$. (Here $\left|a_{0}^{\ell_{0}}\right|=\varepsilon_{0}$.) Since $p_{0}\left(\xi_{i}\right) \in K^{\circ}[x]$ is monic and $\left|a_{0}\right| \leq 1, \mathcal{O}_{K}^{\dagger}\left(\varphi_{0}\right)$ is a finite extension of $A_{1,0}^{\dagger}$ (in the variable $\eta_{1}$ ) and hence $p_{0}^{\prime}\left(\xi_{1}\right)$ is integral over $A_{1,0}^{\dagger}$. If $\square_{0}^{\prime}$ 
is $\leq$ then $\left(p_{0}^{\prime}\right)^{\ell_{0}^{\prime}} / a_{0}^{\prime}$ is power bounded and hence, by Proposition 5.2.12, strongly power bounded in $\mathcal{O}_{K}^{\dagger}\left(\varphi_{0}\right) \subset \mathcal{O}_{K}^{\dagger}(\varphi)$; and if $\square_{0}^{\prime}$ is $<$ then $\left(p_{0}^{\prime}\right)^{\ell_{0}^{\prime}} / a_{0}^{\prime}$ is topologically nilpotent and hence, by Proposition 5.2.12 strongly topologically nilpotent in $\mathcal{O}_{K}^{\dagger}\left(\varphi_{0}\right) \subset \mathcal{O}_{K}^{\dagger}(\varphi)$. The result follows from Corollary 5.2.13.

Case 2. $\square_{1}$ is $<$ and the " $K$-hole" $\mathcal{H}_{1}^{\prime}=\left\{x:\left|p_{1}^{\prime}(x)\right| \square_{1}^{\prime} \varepsilon_{1}^{\prime}\right\}$ is contained in the " $K$-hole" $\mathcal{H}_{1}=\left\{x:\left|p_{1}(x)\right| \leq \varepsilon_{1}^{\prime}\right\}$. Let $a \in K$ with $|a|>1$ and consider the annulus formula (cf. $\operatorname{Remark} 5.2 .15) \varphi_{1}:=(|x|<|a|) \wedge\left(\varepsilon_{1}<\left|p_{1}(x)\right|\right)$. Then, using that remark, $\mathcal{O}_{K}^{\dagger}\left(\varphi_{1}\right) \subset$ $\mathcal{O}_{K}^{\dagger}(\varphi)$ and $\varphi_{1}$ is open. Hence by Proposition 5.2.7, $p_{1}^{\prime \ell_{1}^{\prime}} / a_{1}^{\prime}$ is a unit in $\mathcal{O}_{K}^{\dagger}\left(\varphi_{1}\right)$ and thus also in $\mathcal{O}_{K}^{\dagger}(\varphi)$. Thus $a_{1}^{\prime} / p_{1}^{\prime \ell_{1}^{\prime}} \in \mathcal{O}_{K}^{\dagger}\left(\varphi_{1}\right)$, which is topologically nilpotent, is strongly topologically nilpotent by Proposition 5.2.12

The other cases are similar.

From Proposition 5.3.2 we have

Corollary 5.3.3. If $\varphi$ is a $K$-annulus formula then $\mathcal{O}_{K}^{\dagger}(\varphi)$ depends only on the underlying $K$-annulus $\mathcal{U}_{\varphi}$ and not on the particular presentation. If $\varphi, \psi$ are two $K$-annulus formulas and $\mathcal{U}_{\varphi} \subseteq \mathcal{U}_{\psi}$ then $\mathcal{O}_{K}^{\dagger}\left(\mathcal{U}_{\psi}\right) \subseteq \mathcal{O}_{K}^{\dagger}\left(\mathcal{U}_{\varphi}\right)$.

The following proposition is a special case of Corollary 5.3.3. The proof in the linear case is direct and does not use Proposition 5.3.2, so we include it as a concrete example.

Proposition 5.3.4. For a linear annulus formula $\varphi, \mathcal{O}_{K}^{\dagger}(\varphi)$ depends only on $\mathcal{U}_{\varphi}$ and not on the particular description $\varphi$.

Proof. If $\alpha_{0}^{\prime}$ is another center of the disc $\left\{x:\left|x-\alpha_{0}\right| \square_{0} \varepsilon_{0}\right\}$ then $\left(x-\alpha_{0}^{\prime}\right) / \varepsilon_{0}=$ $\left(x-\alpha_{0}\right) / \varepsilon_{0}+\left(\alpha_{0}^{\prime}-\alpha_{0}\right) / \varepsilon$, and $\left(\alpha_{0}^{\prime}-\alpha_{0}\right) / \varepsilon_{0} \leq 1$ if $\square_{0}$ is $\leq$, and $\left(\alpha_{0}^{\prime}-\alpha_{0}\right) / \varepsilon_{0}<1$ if $\square_{0}$ is $<$. Similarly for a hole $\left\{x: \varepsilon_{1} \square_{1}\left|x-\alpha_{1}\right|\right\}$, if $\alpha_{1}^{\prime}$ is another center then we have

$$
\frac{\varepsilon_{1}}{x-\alpha_{1}^{\prime}}=\frac{\varepsilon_{1}}{\left(x-\alpha_{1}\right)+\left(\alpha_{1}-\alpha_{1}^{\prime}\right)}=\frac{\varepsilon_{1}}{x-\alpha_{1}}\left(1-\frac{\alpha_{1}^{\prime}-\alpha_{1}}{x-\alpha_{1}}\right)^{-1} \text {. }
$$

If $\square_{1}$ is $\leq$ then $\left|\alpha_{1}^{\prime}-\alpha_{1}\right|<\varepsilon_{1}$ and if $\square_{1}$ is $<$ then $\left|\alpha_{1}^{\prime}-\alpha_{1}\right| \leq \varepsilon_{1}$.

The following lemma is a small extension of [CLR1, Lemma 3.11].

Lemma 5.3.5. Every $K$-annulus is a finite union of

(i) thin $K$-annuli with good descriptions of the form

$$
\left|p_{0}(x)\right| \leq \varepsilon_{0} \wedge \varepsilon_{0} \leq\left|p_{0}(x)\right| \wedge \bigwedge_{i=2}^{n} \varepsilon_{i} \leq\left|p_{i}(x)\right|,
$$

(ii) simple Laurent $K$-annuli (cf. Definition 5.1.6) (with good descriptions of the form $\left.\left|p_{0}(x)\right|<\varepsilon_{0} \wedge \varepsilon_{1}<\left|p_{1}(x)\right|\right)$

(iii) open $K$-discs (with good descriptions of the form $\left|p_{0}(x)\right|<\varepsilon_{0}$ ). 
Next we prove Mittag-Leffler type decompositions for $K$-annuli of some special types, namely discs (Lemma 5.3.6) and thin annuli (Lemma 5.3.7).

Lemma 5.3.6. Let $\mathcal{U}$ be a $K$-disc and let $0 \neq f \in \mathcal{O}_{K}^{\dagger}(\mathcal{U})$. Then there is a unique monic polynomial $P(x) \in K[x]$ and strong unit $E \in \mathcal{O}_{K}^{\dagger}(\mathcal{U})$ such that $f=P(x) \cdot E$. In particular, the mapping $f \mapsto f^{\sigma}$ in injective.

Proof. The case of a linear $K$-disc is immediate by Weierstrass Preparation, since in that case $f=\sum a_{i}\left(\frac{x-\alpha}{\varepsilon}\right)^{i}$ with the $a_{i}$ constants.

For $\mathcal{U}$ an arbitrary $K$-disc we write by Weierstrass Division

$$
f=\sum_{i} a_{i}(x)\left(\frac{p(x)}{\varepsilon}\right)^{i}
$$

where the $a_{i}$ have degrees $<\operatorname{deg}(p(x))$ and hence are either zero or strong units on $\mathcal{U}$. Considering $f$ on each of the $K_{\text {alg }}$-discs into which $\mathcal{U}$ decomposes, we see that $\|f\|_{\text {sup }}=$ $\max _{i}\left\|a_{i}\right\|_{\text {sup. }}$. Hence $f \mapsto f^{\sigma}$ is injective and from the linear case we see that $f^{\sigma}$ has only finitely many zeros. The result now follows from Proposition 5.3.1 and the Nullstellensatz 5.2.7. A unit on a disc is necessarily a strong unit.

Lemma 5.3.7. If $\mathcal{U}_{\varphi}$ is a thin $K$-annulus described by a good $K$-annulus formula

$$
\varphi:=\left|p_{0}(x)\right| \leq \varepsilon_{0} \wedge \varepsilon_{0} \leq\left|p_{0}(x)\right| \wedge \bigwedge_{i=2}^{n} \varepsilon_{i} \leq\left|p_{i}(x)\right|
$$

and $0 \neq f \in \mathcal{O}_{K}^{\dagger}\left(\mathcal{U}_{\varphi}\right)$, there is a monic $P(x) \in K[x]$ all of whose zeros lie in $\mathcal{U}_{\varphi}$, a strong unit $E \in \mathcal{O}_{K}^{\dagger}\left(\mathcal{U}_{\varphi}\right)$ and integers $n_{i}$ such that

$$
f=P(x) \cdot \prod_{i=1}^{n} p_{i}(x)^{n_{i}} \cdot E .
$$

Furthermore, this representation is unique. The mapping $f \mapsto f^{\sigma}$ is injective.

Proof. Write

$$
f=\sum_{i, j, v} a_{i j v}(x)\left(\frac{p_{0}(x)}{\varepsilon_{0}}\right)^{i}\left(\frac{\varepsilon_{0}}{p_{0}(x)}\right)^{j}\left(\frac{\varepsilon}{p}\right)^{v^{\prime}}
$$

where $v^{\prime}=\left(v_{2}, \ldots, v_{n}\right)$ is a multi-index and $(\varepsilon / p)^{v^{\prime}}=\left(\varepsilon_{2} / p_{2}\right)^{v_{2}} \cdots\left(\varepsilon_{n} / p_{n}\right)^{v_{n}}$. (We are implicitly assuming that $\varepsilon_{i} \in|K|-\{0\}$. The general case, with $\ell_{i}>1$, is only notationally more cumbersome. We are also abusing notation by using $\varepsilon_{i}$ for both an element of $|K|$ and an element of $K$ of that size.) Let $p(x):=\prod_{i=1}^{n} p_{i}(x), \varepsilon:=\prod_{i=1}^{n} \varepsilon_{i}$ and $N:=\sum_{i=1}^{n} n_{i}$, where $n_{i}$ is the degree of $p_{i}(x)$. (We are taking $p_{1}(x)=p_{0}(x)$ and $\varepsilon_{1}=\varepsilon_{0}$.)

Observe that $\mathcal{U}_{\varphi}$ is also defined by the condition $|p(x)|=\varepsilon$ and that $p(x) \in K^{\circ}[x]$ is monic. (Outside the $K$-disc $\left\{x:\left|p_{0}(x)\right| \leq \varepsilon_{0}\right\}$ we have $|p(x)|>\varepsilon$ and in the holes of $\mathcal{U}_{\varphi}$ 
we have $|p(x)|<\varepsilon$.) Hence, by Proposition 5.3.2 (in fact a small extension to the case that $\psi$ is presented by $|p(x)|=\varepsilon$ ) we have

$$
f=\sum_{i j} a_{i j}(x)\left(\frac{p(x)}{\varepsilon}\right)^{i}\left(\frac{\varepsilon}{p(x)}\right)^{j} .
$$

Dividing $\sum a_{i j} \xi_{0}^{i} \xi_{1}^{j}$ by $p(x)-\varepsilon \xi_{0}$, which is regular in $x$ of degree $N$, we may assume that the $a_{i j}(x)$ all have degrees $<N$.

This representation is far from canonical because of the terms $(p(x) / \varepsilon)^{i}(\varepsilon / p(x))^{j}$. If our Weierstrass system were strong (cf. Section 5.6) we could work with the canonical representation $f=f_{1}(x, p / \varepsilon)+(\varepsilon / p) f_{2}(x, \varepsilon / p)$, with coefficients of degrees less than $N$. The proof is much easier in this case-see Remark 5.3.9 below. In the absence of the strongness assumption, we proceed as follows to get a canonical representation. We have

$$
f \equiv \sum_{i, j} a_{i j}(x) \xi_{0}^{i} \xi_{1}^{j} \bmod \left(\varepsilon \xi_{0}-p(x), \xi_{1} p(x)-\varepsilon\right) .
$$

Doing Weierstrass division by $\xi_{0} \xi_{1}-1$ in the variables $\xi_{0}$ and $\xi_{0}-\xi_{1}$ (i.e. writing $\eta:=$ $\xi_{0}-\xi_{1}$, so $\xi_{1}=\eta+\xi_{0}$ and $\xi_{0} \xi_{1}-1=\xi_{0}^{2}+\xi_{0} \eta-1$, which is regular in $\xi_{0}$ of degree 2 ) we obtain

$$
f \equiv \sum_{i,} b_{i}(x)\left(\xi_{0}-\xi_{1}\right)^{i}+\xi_{0} \sum_{i} c_{i}(x)\left(\xi_{0}-\xi_{1}\right)^{i}
$$

modulo $\left(\varepsilon \xi_{0}-p(x), \xi_{1} p(x)-\varepsilon_{0}, \xi_{0} \xi_{1}-1\right)$, where the $b_{i}$ and $c_{i}$ have degrees less than $N$. Next we observe that for representations of this form

$$
\|f\|_{\sup }=\max _{i}\left\{\left\|b_{i}\right\|_{\text {sup }},\left\|c_{i}\right\|_{\text {sup }}\right\}
$$

and that $\|\cdot\|_{\text {sup }}$ is a multiplicative norm. From the Strong Noetherian Property there are only finitely many "biggest" terms. Consider

$$
\left|b_{i}(x)\left(\frac{p(x)}{\varepsilon}-\frac{\varepsilon}{p(x)}\right)^{i}\right| \text { and }\left|c_{j}(x) \frac{p(x)}{\varepsilon}\left(\frac{p(x)}{\varepsilon}-\frac{\varepsilon}{p(x)}\right)^{j}\right|
$$

for $x$ "just" outside the outer edge of $\mathcal{U}_{\varphi}$ and "just" inside the holes in $\mathcal{U}_{\varphi}$, and the fact that the degrees of the $b_{i}, c_{j}$ are $<N$, to see that there cannot be any cancellation among "biggest" terms. This proves injectivity.

By the Strong Noetherian Property and Corollary 4.5.9 we can write

$$
f=\sum_{i \leq s} b_{i}(x)\left(\frac{p(x)}{\varepsilon}-\frac{\varepsilon}{p(x)}\right)^{i}\left(1+g_{i}\right)+\frac{p(x)}{\varepsilon} \sum_{i \leq s} c_{i}(x)\left(\frac{p(x)}{\varepsilon}-\frac{\varepsilon}{p(x)}\right)^{i}\left(1+g_{i}^{\prime}\right)
$$

with the $g_{i}, g_{i}^{\prime}$ in $\mathcal{O}_{K}^{\circ}(\mathcal{U})$.

Let

$$
R:=\sum_{i \leq s} b_{i}(x)\left(\frac{p(x)}{\varepsilon}-\frac{\varepsilon}{p(x)}\right)^{i}+\frac{p(x)}{\varepsilon} \sum_{i \leq s} c_{i}(x)\left(\frac{p(x)}{\varepsilon}-\frac{\varepsilon}{p(x)}\right)^{i} .
$$


Then $\|f(x)-R(x)\|_{\text {sup }}<\|R\|_{\text {sup }}=\|f\|_{\text {sup }}$, and hence, except on finitely many discs $\mathcal{U}_{j}^{\prime}$ contained in $\mathcal{U}$ (around the zeros of $R$ ), we have for $x \in \mathcal{U}$ (i.e. for all $x \in \mathcal{U} \backslash \bigcup_{j} \mathcal{U}_{j}^{\prime}$ ) that $|f(x)-R(x)|<|f(x)|=|R(x)|=\|f\|_{\text {sup }}=\|R\|_{\text {sup }}$. Clearly, $f^{\sigma}$ is not identically zero on any of these discs $\mathcal{U}_{j}^{\prime}$. Hence, by Lemma 5.3.6 $f^{\sigma}$ has only finitely many zeros in these discs. Hence, $f$ has only finitely many zeros and by Proposition 5.3.1 we may divide by a suitable polynomial $P(x) \in K[x]$ and reduce to the case that $f$ has no zeros and hence, by the Nullstellensatz (Proposition 5.2.7), is a unit. Since a unit on a disc is a strong unit, we see that now $|f(x)|=\|f\|_{\text {sup }}$ for all $x \in \mathcal{U}$. Hence $R$ is also a unit, and we have $f=R \cdot E$ for $E$ a unit in $\mathcal{O}_{K}(\mathcal{U})$. Since $|f(x)-R(x)|<|f(x)|$ for all $x \in \mathcal{U}, E$ is a strong unit. Note that $R(x)$ is a rational function with denominator of the form $\prod p_{i}(x)^{m_{i}}$. The numerator has no zeros in $\mathcal{U}$, since $f$ is a unit. Let $Q(x)$ be an irreducible factor of the numerator. If one of (and hence all of) the zeros of $Q$ lies outside the disc $\left\{x:\left|p_{0}(x)\right| \leq \varepsilon_{0}\right\}, Q$ is a strong unit on $\mathcal{U}$. If one of (and hence all of) the zeros of $Q$ lies inside the hole $\left\{x:\left|p_{i}(x)\right|<\varepsilon_{i}\right\}$, then by iterated use of Lemma 5.3.8 below, there is a strong unit $E^{\prime}$ and an $\ell \in \mathbb{N}$ such that $Q(x)=\left(p_{i}(x)\right)^{\ell} \cdot E^{\prime}$, and this completes the proof of existence. Uniqueness follows from the observation that $P$ is determined by the zeros of $f$ and that $\prod_{i} p_{i}^{n_{i}}$ is a strong unit only when $n_{i}=0$ for all $i$.

Lemma 5.3.8. Let $|p(x)|<\varepsilon$ be a good description of a $K$-disc $\mathcal{H}$, and let $P(x) \in$ $K^{\circ}[x]$ have all its zeros in $\mathcal{H}$. Then there is a strong unit $E$ on the annulus $\mathcal{U}=$ $\{x:|p(x)|=\varepsilon\}$ such that $P(x)=p(x) \cdot q(x) \cdot E$ for some $q(x) \in K[x]$ of degree less than that of $P(x)$. Indeed, the conclusion is true on the whole annulus $\mathcal{U}^{\prime \prime}=\{x:|p(x)| \geq \varepsilon\}$.

Proof. It is sufficient to consider $P$ irreducible. By Euclidean division

$$
P(x)=p(x) \cdot q(x)+r(x),
$$

where $\operatorname{deg} r(x)<\operatorname{deg} p(x)$. Hence either $r(x)=0$ or $r(x)$ is a strong unit on $\mathcal{H}$. $\mathcal{H}$ is also described by $|P(x)|<\varepsilon^{\prime} \leq \varepsilon$, since $\operatorname{deg} P(x) \geq \operatorname{deg} p(x)$. Let $\alpha$ be a zero of $p(x)$. Then $\varepsilon \geq \varepsilon^{\prime}>|P(\alpha)|=|r(\alpha)|$. So $\|r\|_{\sup _{\mathcal{H}}}<\varepsilon^{\prime}$, where $\|\cdot\|_{\sup _{\mathcal{H}}}$ is the supremum norm on $\mathcal{H}$. Hence also $\|r\|_{\text {sup }_{\mathcal{U}}}<\varepsilon^{\prime}$, where $\|\cdot\|_{\text {sup }_{\mathcal{U}}}$ is the supremum norm on $\mathcal{U}$. Hence $E:=P(x) /(P(x)-r(x))$ is a strong unit on $\mathcal{U}$, i.e.

$$
P(x)=[P(x)-r(x)] E=p(x) q(x) E .
$$

Since the final conclusion of the lemma is not used, we leave its proof to the reader.

Remark 5.3.9. As remarked in the proof of Lemma 5.3.7, the proof is simpler when the Weierstrass system is strong. In that case $f=f_{1}(x, p / \varepsilon)+(\varepsilon / p) f_{2}(x, \varepsilon / p)$. Multiplying by a suitable power of $\varepsilon / p$, dividing by a strong unit (and using the Strong Noetherian Property), we may assume that $f$ is regular in $\varepsilon / p$, of degree $s$, say. Hence, by Weierstrass Preparation, multiplying by a strong unit $E$, we are reduced to the case that $E \cdot p^{s} \cdot f=$ $f_{1}(x, p / \varepsilon)$. This case is the same as that of a disc, handled in Lemma 5.3.6.

We do not use the following

Corollary 5.3.10. We showed above that when $\mathcal{U}$ is thin, and when $\mathcal{U}$ is a $K$-disc, the mapping $f \mapsto f^{\sigma}$ for $f \in \mathcal{O}(\mathcal{U})$ is injective. A similar result, with a similar proof, also holds for simple Laurent annuli. 
We include the following proposition and its corollary for completeness-we do not use it except in the proof of Theorem 5.6.5. The slightly weaker version of that theorem in which $f$ is replaced by $f^{\sigma}$ does not require Proposition 5.3.11

Proposition 5.3.11. Let $\varphi$ be a $K$-annulus formula, and let $F$ be an algebraic extension of $K$ over which all the polynomials in $\varphi$ split and containing the $a_{i}$ (as above $a_{i}^{\ell_{i}}=\varepsilon_{i}$ ). Then:

(i) $\mathcal{O}_{K}^{\dagger}(\varphi) \hookrightarrow \mathcal{O}_{K}^{\dagger}(\varphi) \otimes_{K} F$ is a faithfully flat extension.

(ii) $\mathcal{O}_{K}^{\dagger}(\varphi) \otimes_{K} F \simeq \bigoplus_{i=1}^{N} \mathcal{O}_{F}^{\dagger}\left(\mathcal{U}_{i}\right)$ where the $\mathcal{U}_{i}$ are the linear $K_{\text {alg-annuli which make }}$ up $\mathcal{U}_{\varphi}$ (see Lemma 5.2(ii)).

Proof. Since $A_{m, n}(F)$ is an integral extension of $A_{m, n}(K)$, (i) is immediate. Part (ii) follows by induction and rescaling from the lemma below.

Lemma 5.3.12. Let $g=\prod_{i=1}^{n}\left(x-\alpha_{i}\right) \in F[x]$ and assume for all $i, j$ that $\left|\alpha_{i}\right|=1$, $\left|\alpha_{i}-\alpha_{j}\right|=1$ if $i \neq j$ and that on $\mathcal{U}_{\varphi},|g(x)| \square \varepsilon$, where either $\square$ is $\leq$ and $\varepsilon<1$ or $\square$ is $<$ and $\varepsilon \leq 1$. Then in $\mathcal{O}_{F}^{\dagger}(\varphi)$ (indeed in $\mathcal{O}_{F}^{\dagger}\left(\varphi_{0}\right)$ ),

$$
g\left(\xi_{1}\right)-\varepsilon z_{1}=\prod_{i=1}^{n}\left(\xi_{1}-\alpha_{i}-h_{i}\right)
$$

where $h_{i} \in A_{2,0}^{\circ}$ or $A_{1,1}^{\circ}$ (according as $\square$ is $\leq$ or $<$ ).

Proof. As above $z_{1}=g\left(\xi_{1}\right) / \varepsilon$ is strongly power bounded (resp. topologically nilpotent). Consider the case that $\square$ is $\leq$. The other case is similar. Let $H$ be a new type 1 variable. By Taylor's theorem we have

$$
\begin{aligned}
\left(g-\varepsilon z_{1}\right)\left(\alpha_{1}+\varepsilon H\right) & =g\left(\alpha_{1}\right)-\varepsilon z_{1}+g^{\prime}\left(\alpha_{1}\right) \varepsilon H+\frac{g^{\prime \prime}\left(\alpha_{1}\right)}{2 !}(\varepsilon H)^{2}+\cdots \\
& =\varepsilon z_{1}+g^{\prime}\left(\alpha_{1}\right) \varepsilon H+\frac{g^{\prime \prime}\left(\alpha_{1}\right)}{2 !}(\varepsilon H)^{2}+\cdots \\
& =\varepsilon\left[z_{1}+g^{\prime}\left(\alpha_{1}\right) H+\frac{g^{\prime \prime}\left(\alpha_{1}\right)}{2 !} \varepsilon H^{2}+\cdots\right] .
\end{aligned}
$$

Observe that $\left|g^{\prime}\left(\alpha_{1}\right)\right|=1$, hence by Weierstrass Preparation there is an $A \in \mathcal{O}_{F}\left(\varphi_{0}\right)$ such that

$$
\left(g-\varepsilon z_{1}\right)\left(\alpha_{1}+\varepsilon H\right)=g^{\prime}\left(\alpha_{1}\right)[H-A] Q .
$$

Then $\alpha_{1}+\varepsilon A$ is the required zero of $g-\varepsilon z_{1}$.

\subsection{Linear $K$-annuli}

In this subsection we prove some basic results (in particular Proposition 5.4.5 for linear $K$-annuli. In subsequent subsections we will extend several of these results to general $K$-annuli. The following lemma, which is a special case of Lemma 5.3.6, is immediate by Weierstrass Preparation. 
Lemma 5.4.1. Let $\mathcal{U}$ be a linear $K$-disc and let $f(x) \in \mathcal{O}(\mathcal{U})$. There is a polynomial $P(x) \in K[x]$ all of whose zeros lie in $\mathcal{U}$ and a strong unit $E \in \mathcal{O}(\mathcal{U})$ such that

$$
f(x)=P(x) E(x) .
$$

The case of a linear Laurent annulus is a little more complicated.

Lemma 5.4.2. Let $\mathcal{U}$ be a linear Laurent $K$-annulus described by an annulus formula $\varepsilon_{1}<|x-\alpha|<\varepsilon_{0}$ and let $0 \neq f(x) \in \mathcal{O}(\mathcal{U})$. There is a monic polynomial $P(x)$ all of whose zeros lie in $\mathcal{U}$, an integer $n$ and a strong unit $E \in \mathcal{O}(\mathcal{U})$ such that

$$
f=P(x)(x-\alpha)^{n} E .
$$

Proof. As in the proof of Lemma 5.3.7 doing Weierstrass division by $x-\alpha-\varepsilon_{0}\left(\frac{x-\alpha}{\varepsilon_{0}}\right)$, we may assume that

$$
f=\sum_{i, j} a_{i j}\left(\frac{x-\alpha}{\varepsilon_{0}}\right)^{i}\left(\frac{\varepsilon_{1}}{x-\alpha}\right)^{j}
$$

where the $a_{i j}$ are of degree $<1$, i.e. constants. Such a representation is again far from canonical—for example we could have $f=\left(\frac{\varepsilon_{1}}{\varepsilon_{0}}\right)\left(\frac{x-\alpha}{\varepsilon_{0}}\right)\left(\frac{\varepsilon_{1}}{x-\alpha}\right)-\left(\frac{x-\alpha}{\varepsilon_{0}}\right)^{2}\left(\frac{\varepsilon_{1}}{x-\alpha}\right)^{2}$, which is actually the zero function.

As in the proof of Lemma 5.3.7 (using the relation $\rho_{0} \rho_{1}-\varepsilon_{1} / \varepsilon_{0}=0$ instead of $\xi_{0} \xi_{1}-1=0$ ) we can write

$$
f=\sum_{i} b_{i}\left(\frac{x-\alpha}{\varepsilon_{0}}-\frac{\varepsilon_{1}}{x-\alpha}\right)^{i}+\frac{x-\alpha}{\varepsilon_{0}} \sum_{i} c_{i}\left(\frac{x-\alpha}{\varepsilon_{0}}-\frac{\varepsilon_{1}}{x-\alpha}\right)^{i}
$$

where the $b_{i}, c_{i}$ are constants.

Using the Strong Noetherian Property, Theorem 4.2.15 and Corollary 4.5.9 we see that there are only finitely many biggest $b_{i}$ and $c_{i}$. Among the biggest terms let those of lowest degrees in the two sums be $b_{i}\left(\frac{x-\alpha}{\varepsilon_{0}}-\frac{\varepsilon_{1}}{x-\alpha}\right)^{i}$ and $c_{j} \frac{x-\alpha}{\varepsilon_{0}}\left(\frac{x-\alpha}{\varepsilon_{0}}-\frac{\varepsilon_{0}}{x-\alpha}\right)^{j}$. Of course, one may be missing. If $i>j+1$, after dividing by $b_{i}, f$ is regular in $(x-\alpha) / \varepsilon_{0}$ of degree $i$. Similarly, if $i<j+1$, after dividing by $c_{j}, f$ is regular in $(x-\alpha) / \varepsilon_{0}$ of degree $j+1$. If $i=j+1$, after dividing by $b_{i}, f$ is regular in $\varepsilon_{1} /(x-\alpha)$ of degree $i$. Hence we may assume that $f$ is regular in either $(x-\alpha) / \varepsilon_{0}$ or $\varepsilon_{1} /(x-\alpha)$. Assume that $f$ is regular in $(x-\alpha) / \varepsilon_{0}$. The other case is similar. By Weierstrass Preparation (multiplying by a strong unit) we may assume that $f$ is actually a polynomial in $(x-\alpha) / \varepsilon_{0}$, of degree $s$, say. Hence $f \cdot\left(\frac{x-\alpha}{\varepsilon_{0}}\right)^{-s}=g\left(\frac{\varepsilon_{1}}{x-\alpha}\right)$, and a second use of Weierstrass Preparation completes the proof.

For linear almost thin annuli we have the following lemma.

Lemma 5.4.3. Let $\mathcal{U}$ be an almost thin linear $K$-annulus with annulus formula either

$$
\left|x-a_{0}\right| \leq \varepsilon^{\prime} \wedge \bigwedge_{i=0}^{n}\left|x-a_{i}\right| \geq \varepsilon
$$


where $\varepsilon^{\prime}>\varepsilon$, the $\left|a_{i}\right| \geq \varepsilon$ and $\left|a_{i}-a_{j}\right|=\varepsilon$ for $i \neq j$, or

$$
\left|x-a_{0}\right| \geq \varepsilon \wedge \bigwedge_{i=0}^{n}\left|x-a_{i}\right| \leq \varepsilon^{\prime} \wedge \bigwedge_{i=1}^{n}\left|x-a_{i}\right| \geq \varepsilon^{\prime}
$$

where $\varepsilon^{\prime}>\varepsilon$, the $\left|a_{i}\right| \geq \varepsilon^{\prime}$ and $\left|a_{i}-a_{j}\right|=\varepsilon^{\prime}$ for $i \neq j$. Let $f \in \mathcal{O}^{\dagger}(\mathcal{U})$ with $f^{\sigma} \neq 0$. There is an $\varepsilon^{\prime \prime}$ with $\varepsilon<\varepsilon^{\prime \prime}<\varepsilon^{\prime}$ such that, denoting by $\mathcal{U}^{\prime \prime}$ the almost thin annulus defined, respectively, by the annulus formula

$$
\left|x-a_{0}\right| \leq \varepsilon^{\prime \prime} \wedge \bigwedge_{i=0}^{N}\left|x-a_{i}\right| \geq \varepsilon,
$$

or

$$
\left|x-a_{0}\right| \geq \varepsilon^{\prime \prime} \wedge \bigwedge_{i=0}^{n}\left|x-a_{i}\right| \leq \varepsilon^{\prime} \wedge \bigwedge_{i=1}^{n}\left|x-a_{i}\right| \geq \varepsilon^{\prime},
$$

there is a monic polynomial $P(x)$ all of whose zeros lie in the corresponding thin annulus $\mathcal{U}_{t}^{\prime \prime}=\mathcal{U}_{t}$ (Definition 5.1.6(iii)), integers $n_{i}$ and a strong unit $E \in \mathcal{O}^{\dagger}\left(\mathcal{U}^{\prime \prime}\right)$ such that

$$
\left.f^{\sigma}\right|_{\mathcal{U}^{\prime \prime}}=P(x) \cdot \prod_{i=0}^{n}\left(x-a_{i}\right)^{n_{i}} \cdot E^{\sigma} .
$$

The polynomial $P$, the integers $n_{i}$ and the strong unit $E$ are unique.

Proof. By Lemma 5.1.8 the two cases are equivalent, so we need only consider the first case. Then the annulus $\mathcal{U}$ is also defined by the formula

$$
|p(x)| \geq \varepsilon^{n+1} \wedge|p(x)| \leq\left(\varepsilon^{\prime}\right)^{n+1}
$$

where

$$
p(x):=\prod_{i=0}^{n}\left(x-a_{i}\right) .
$$

Hence, by Propositions 5.2.12 and 5.2.13 or 5.3.2 and Weierstrass Division, we can write

$$
f(x)=\sum_{i=0}^{n} \sum_{j, k \geq 0} a_{i j k}^{\prime} x^{i}\left(\frac{p(x)}{\left(\varepsilon^{\prime}\right)^{n+1}}\right)^{j}\left(\frac{\varepsilon^{n+1}}{p(x)}\right)^{k}
$$

where the $a_{i j k}^{\prime}$ are in $K^{\circ}$. Rescaling, we may assume that $\varepsilon^{\prime}=1$, so

$$
f(x)=\sum_{i=0}^{n} \sum_{j, k \geq 0} a_{i j k} x^{i}(p(x))^{j}\left(\frac{\varepsilon^{n+1}}{p(x)}\right)^{k}
$$

where the $a_{i j k}$ are in $K^{\circ}$. As in the proof of Lemma 5.3.7, using the relations

$$
p(x) \cdot \frac{\varepsilon^{n+1}}{p(x)}=\varepsilon^{n+1}
$$

(i.e. $\xi_{1} \cdot \xi_{2}=\varepsilon^{n+1}, \eta=\xi_{1}+\xi_{2}$ and $\xi_{1}^{2}-\eta \xi_{1}-\varepsilon^{n+1}=0$ ) we have 


$$
\begin{aligned}
f(x) & =\sum_{i=0}^{n} \sum_{j=0}^{\infty} a_{i j} x^{i}\left(p(x)+\frac{\varepsilon^{n+1}}{p(x)}\right)^{j}+p(x) \sum_{i=0}^{n} \sum_{j=0}^{\infty} b_{i j} x^{i}\left(p(x)+\frac{\varepsilon^{n+1}}{p(x)}\right)^{j} \\
& =\sum_{i=0}^{n} x^{i}\left[\sum_{j=0}^{\infty} a_{i j}\left(p(x)+\frac{\varepsilon^{n+1}}{p(x)}\right)^{j}+p(x) \sum_{j=0}^{\infty} b_{i j}\left(p(x)+\frac{\varepsilon^{n+1}}{p(x)}\right)^{j}\right] \\
& =\sum_{i=0}^{n} x^{i} h_{i}(x),
\end{aligned}
$$

say. Without loss of generality we may assume $\|f\|=1$, i.e. $\max \left\{\left|a_{i j}\right|,\left|b_{i j}\right|\right\}=1$.

Case (i): some $h_{i}$ with $\left|h_{i}\right|=1$ is regular in $p(x)$, and hence also in $x$. Then $f(x)$ is regular in $x$ and by Weierstrass Preparation we can write

$$
f=U \cdot\left[x^{s}+A_{1} x^{s-1}+\cdots+A_{s}\right]
$$

where each $A_{j}$ is a power series in $\varepsilon^{n+1} / p(x)$ and $U$ is a (strong) unit. Hence, by Euclidean division by $p(x)$ we are reduced to the case that

$$
f(x)=\sum_{i=0}^{n} \sum_{j=0}^{\infty} a_{i j} x^{i}\left(\frac{\varepsilon^{n+1}}{p(x)}\right)^{j} .
$$

There are finitely many terms that are biggest in the supremum norm on $\mathcal{U}_{t}$, the corresponding thin annulus defined by

$$
\left|x-a_{0}\right| \leq \varepsilon \wedge \bigwedge_{i=0}^{n}\left|x-a_{i}\right| \geq \varepsilon .
$$

These occur for $j<L$, say. Since these terms are all of different degrees $i-(n+1) j$, $0 \leq i \leq n, 0 \leq j<L$, there can be no cancellation in supremum norm, and exactly one of these terms will be biggest on an annulus $\mathcal{U}^{*}$ defined by $\varepsilon^{n+1}<|p(x)| \leq\left(\varepsilon^{\prime \prime}\right)^{n+1}$ for some $\varepsilon<\varepsilon^{\prime \prime}<1$. Hence, shrinking $\varepsilon^{\prime \prime}$ if necessary, we may assume that (i) $f(x)=$ $R(x)+g(x)$ where $R(x)$ is a rational function whose denominator is a power of $p(x)$, (ii) $R(x)$ has all its zeros $\beta_{i}$ in $\mathcal{U}_{t}$, and (iii) $|R(x)|>|g(x)|$ outside the open discs of radius $\varepsilon$ around the $\beta_{i}$. Applying Proposition 5.3.1 we may further assume that $f$ has no zeros in $\mathcal{U}^{\prime \prime}$ and hence is a strong unit on every disc contained in $\mathcal{U}^{\prime \prime}$. Then $R(x)$ is a unit, and $f \cdot R^{-1}=1+R^{-1} \cdot g$ is a strong unit and the proof of existence is complete in this case. Uniqueness follows exactly as in the proof of Lemma 5.3.7 using Lemma 5.3.8.

Case (ii): no $h_{i}$ with $\left\|h_{i}\right\|=1$ is regular in $p(x)$. Hence, as in the proof of Lemma 5.3.7, all are "regular" in $\varepsilon^{n+1} / p(x)$. Then the "biggest" terms in the supremum norm on $\mathcal{U}_{t}$ are all of the form $a_{i j} x^{i}\left(\varepsilon^{n+1} / p(x)\right)^{j}$ with $0 \leq i \leq n$ and $0 \leq j \leq L$ for some $L \in \mathbb{N}$ and $\left|a_{i j}\right|=1$. Indeed,

$$
\left\|a x^{i}\left(\frac{\varepsilon^{n+1}}{p(x)}\right)^{j}(p(x))^{k}\right\|_{\sup _{\mathcal{U}_{t}}}=|a||\varepsilon|^{i}|\varepsilon|^{(n+1) k}
$$

and hence none of the terms with $k>0$ can be biggest. We now complete the argument as in case (i). 


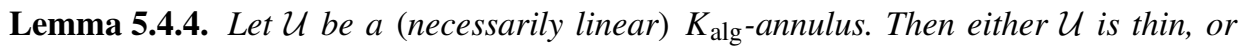
there is a finite rigid (Definition 5.1.6) cover of $\mathcal{U}$ by $K_{\mathrm{alg}}$-discs, almost thin $K_{\mathrm{alg}}$-annuli and open Laurent $K_{\mathrm{alg}}$-annuli $\mathcal{U}_{i}$. Indeed, we can ensure that if each almost thin annulus

$$
\left|x-a_{0}\right| \leq \varepsilon^{\prime} \wedge \bigwedge_{i=0}^{n}\left|x-a_{i}\right| \geq \varepsilon
$$

where $\varepsilon^{\prime}>\varepsilon$, the $\left|a_{i}\right| \geq \varepsilon$ and $\left|a_{i}-a_{j}\right|=\varepsilon$ for $i \neq j$, or

$$
\left|x-a_{0}\right| \geq \varepsilon \wedge \bigwedge_{i=0}^{n}\left|x-a_{i}\right| \leq \varepsilon^{\prime} \wedge \bigwedge_{i=1}^{n}\left|x-a_{i}\right| \geq \varepsilon^{\prime}
$$

where $\varepsilon^{\prime}>\varepsilon$, the $\left|a_{i}\right| \geq \varepsilon^{\prime}$ and $\left|a_{i}-a_{j}\right|=\varepsilon^{\prime}$ for $i \neq j$ in the cover is replaced by $a$ "thinner" annulus

$$
\left|x-a_{0}\right| \leq \varepsilon^{\prime \prime} \wedge \bigwedge_{i=0}^{N}\left|x-a_{i}\right| \geq \varepsilon
$$

or

$$
\left|x-a_{0}\right| \geq \varepsilon^{\prime \prime} \wedge \bigwedge_{i=0}^{n}\left|x-a_{i}\right| \leq \varepsilon^{\prime} \wedge \bigwedge_{i=1}^{n}\left|x-a_{i}\right| \geq \varepsilon^{\prime}
$$

respectively, for any $\varepsilon^{\prime \prime}$ with $\varepsilon<\varepsilon^{\prime \prime}<\varepsilon^{\prime}$, the resulting annuli still form a rigid cover of $\mathcal{U}$.

Proof. The proof is an easy induction on the number of holes in $\mathcal{U}$.

Next we prove the Mittag-Leffler decomposition for analytic functions on $K_{\text {alg }}$-annuli.

Proposition 5.4.5. Let $\mathcal{U}$ be a $K_{\mathrm{alg}}$-annulus and let $\left\{\mathcal{U}_{i}\right\}$ be the rigid cover of $\mathcal{U}$ provided by Lemma 5.4.4 Let $f \in \mathcal{U}$ and assume that for each $i$ we have the unique representation of $f^{\sigma} \mid \mathcal{U}_{i}$ provided by Lemmas 5.4.1 5.4.3 Then, if $f^{\sigma} \neq 0$, it has a unique representation of the form

$$
f^{\sigma}=P(x) \cdot \prod_{i=0}^{n}\left(x-a_{i}\right)^{n_{i}} \cdot E^{\sigma}
$$

where $P$ is a monic polynomial all of whose zeros lie in $\mathcal{U}$, the $a_{i}$ are (preselected) centers of the holes in $\mathcal{U}$, the $n_{i}$ are integers and $E \in \mathcal{O}(\mathcal{U})$ is a strong unit.

Proof. We paste together the representations provided by Lemmas 5.4.1 5.4 .3 for $f^{\sigma} \mid \mathcal{U}_{i}$. Consider the case that $\mathcal{U}_{1}$ is an almost thin annulus with annulus formula

$$
\left|x-a_{0}\right| \geq \varepsilon \wedge \bigwedge_{i=0}^{n}\left|x-a_{i}\right| \leq \varepsilon^{\prime} \wedge \bigwedge_{i=1}^{n}\left|x-a_{i}\right| \geq \varepsilon^{\prime}
$$

and $\mathcal{U}_{2}$ is a Laurent annulus with annulus formula

$$
\varepsilon_{1}<|x-\alpha|<\varepsilon^{\prime}
$$


and that $\varepsilon_{1}<\varepsilon<\varepsilon^{\prime}$, so that $\mathcal{U}_{1}$ and $\mathcal{U}_{2}$ overlap. We may assume by Lemma 5.3.8 that $\alpha=a_{0}$ and that $f^{\sigma}$ has no zeros in the annulus $\varepsilon \leq\left|x-a_{0}\right|<\varepsilon^{\prime}$. Let

$$
f^{\sigma}\left|\mathcal{U}_{1}=P_{1}(x) \cdot \prod_{i=0}^{n}\left(x-a_{i}\right)^{n_{i}} \cdot E_{1}^{\sigma}, \quad f^{\sigma}\right| \mathcal{U}_{2}=P_{2}(x) \cdot\left(x-a_{0}\right)^{m} \cdot E_{2}^{\sigma} .
$$

Then all the zeros of $P_{2}$ lie in the hole $\left|x-a_{0}\right|<\varepsilon$ of $\mathcal{U}_{1}$ and by the uniqueness of the representations and Lemma 5.3.8 we have $n_{0}-m=\operatorname{deg} P_{2}$ and $P_{2}(x) \cdot\left(x-a_{0}\right)^{m-n_{0}}$ is a strong unit on $\mathcal{U}_{1}$. Hence

$$
f^{\sigma} \mid \mathcal{U}_{1} \cup \mathcal{U}_{2}=P_{1}(x) \cdot P_{2}(x) \cdot\left(x-a_{0}\right)^{m} \cdot \prod_{i=1}^{n}\left(x-a_{i}\right)^{n_{i}} \cdot E_{1}^{\sigma} .
$$

The other cases are similar.

\subsection{General $K$-annuli, part 2}

Now let $\mathcal{U}$ be a (not necessarily linear) $K$-annulus, defined by a $K$-annulus formula $\varphi$, let $\mathcal{U}=\bigcup \mathcal{U}_{j}$ be the decomposition of $\mathcal{U}$ into (linear) $K_{\text {alg }}$-annuli, and let $f \in \mathcal{O}_{K}(\varphi)$. After we have (arbitrarily) chosen a center $\alpha_{i j k}$ for each hole in $\mathcal{U}_{j}$ corresponding to the inequality $\varepsilon_{i} \square_{i}\left|p_{i}(x)\right|$, say a zero of $p_{i}$ in that hole, by Proposition [5.4.5, the function $f^{\sigma}$, if it is nonzero, has a (unique) representation on each $\mathcal{U}_{j}$,

$$
f^{\sigma} \mid \mathcal{U}_{j}=P_{j}(x) \cdot \prod_{i, k}\left(x-\alpha_{i j k}\right)^{n_{i j k}} \cdot E_{j}^{\sigma} .
$$

Here $P_{j} \in F[x]$ is monic with zeros only in $\mathcal{U}_{j}$, and $E_{j}$ is a strong unit in $\mathcal{O}_{F}\left(\mathcal{U}_{j}\right) ; F$ is an algebraic extension of $K$ containing the $\alpha_{i j k}$.

Note that for $\ell \neq j$ the function $P_{\ell} \cdot \prod_{i, k}\left(x-\alpha_{i \ell k}\right)^{n_{i \ell k}}$ is a strong unit on $\mathcal{U}_{j}$. Take $R:=\prod_{j}\left[P_{j} \cdot \prod_{i, k}\left(x-\alpha_{i j k}\right)^{n_{i j k}}\right]$. Then $f \cdot R^{-1}$ is a strong unit on each $\mathcal{U}_{j}$ and hence a strong unit on $\mathcal{U}_{\varphi}$. Thus we have the decomposition

$$
f^{\sigma}=P(x) \cdot \prod_{i, j, k}\left(x-\alpha_{i j k}\right)^{n_{i j k}} \cdot E^{\sigma},
$$

where $P \in F[x]$ is monic and has zeros only in $\mathcal{U}_{\varphi}$, and the $\alpha_{i j k}$ are "centers" of the $F$-holes in $\mathcal{U}$. Considering automorphisms of $K_{\text {alg }}$ over $K$ that permute the zeros of $p_{i}$ we see that $n_{i j k}=n_{i j^{\prime} k^{\prime}}$.

By Proposition 5.3.1 we see that $P \in K[x]$.

If $K$ is of equicharacteristic zero, we may assume that each $p_{i}$ occurring in the definition of $\mathcal{U}_{\varphi}$ has only one zero in each disc or hole. Indeed, taking the $p_{i}$ of lowest possible degree (i.e. taking a good description of $\mathcal{U}_{\varphi}$ ) will ensure that, for if $p$ has $m>1$ zeros in each of its holes, say $\alpha_{1}, \ldots, \alpha_{m}$ in one of the holes, then $\beta=m^{-1}\left(\alpha_{1}+\cdots+\alpha_{m}\right)$ is also in that hole and the minimal polynomial of $\beta$ will have lower degree than $p$. Hence in equicharacteristic zero we have in fact

$$
f^{\sigma}=P \cdot \prod_{i} p_{i}^{n_{i}} \cdot E^{\sigma}
$$


where $P \in K[x]$ is monic and has all its zeros in $\mathcal{U}_{\varphi}$, the $n_{i}$ are integers and $E$ is a strong unit. The uniqueness of this representation follows from uniqueness in the linear case. Hence we have proved Theorem 5.5.2 in the equicharacteristic zero case. To obtain the similar result in the general (nonequicharacteristic-zero) case will take a little more work.

Definition 5.5.1. Let $\mathcal{U}_{\varphi}$ be an annulus and let $f \in \mathcal{O}\left(\mathcal{U}_{\varphi}\right)$.

(i) We say that the thin annulus $\mathcal{U}_{\psi}$ surrounds the hole $\mathcal{H}_{i}=\left\{x:\left|p_{i}(x)\right|<\varepsilon_{i}\right\}$ in $\mathcal{U}_{\varphi}$ if $\mathcal{H}_{i}$ is a hole of $\mathcal{U}_{\psi}$ and $\mathcal{U}_{\psi} \subset \mathcal{U}_{\varphi}$.

(ii) We say that the thin annulus $\mathcal{U}_{\psi}$ surrounds the hole $\mathcal{H}_{i}^{\prime}=\left\{x:\left|p_{i}(x)\right| \leq \varepsilon_{i}\right\}$ in $\mathcal{U}_{\varphi}$ if $\mathcal{U}_{\psi}=\left\{x:\left|p_{i}(x)\right|=\delta\right\} \subset \mathcal{U}_{\varphi}, \varepsilon_{i}<\delta$ and $f$ has no zeros in the annulus $\left\{x: \varepsilon_{i}<\left|p_{i}(x)\right| \leq \delta\right\}$ and no other hole of $\mathcal{U}_{\varphi}$ is contained in the hole of $\mathcal{U}_{\psi}$.

(iii) We say that the Laurent annulus $\mathcal{U}_{\psi}$ surrounds the hole $\mathcal{H}_{i}^{\prime}=\left\{x:\left|p_{i}(x)\right| \leq \varepsilon_{i}\right\}$ in $\mathcal{U}_{\varphi}$ if $\mathcal{U}_{\psi}=\left\{x: \varepsilon_{i}<\left|p_{i}(x)\right|<\delta\right\} \subset \mathcal{U}_{\varphi}, \varepsilon_{i}<\delta$ and $f$ has no zeros in the annulus $\left\{x: \varepsilon_{i}<\left|p_{i}(x)\right|<\delta\right\}$.

It is clear that if $\mathcal{U}_{\varphi}$ is a $K$-annulus then for each of the holes of $\mathcal{U}_{\varphi}$ there is a thin $K$ annulus surrounding that hole, and if the hole is of the form $\mathcal{H}_{i}^{\prime}=\left\{x:\left|p_{i}(x)\right| \leq \varepsilon_{i}\right\}$ there is a Laurent annulus surrounding that hole in $\mathcal{U}_{\varphi}$. We will complete the proof of Theorem 5.5.2 by comparing the representations we have obtained above for $f$ on $\mathcal{U}_{\varphi}$ but over $F$ (equation (5.2) with the representations we have from Lemma 5.3.7 for $f$ on these thin annuli surrounding the holes of $\mathcal{U}_{\varphi}$.

Theorem 5.5.2 (Mittag-Leffler Decomposition). Let

$$
\varphi:=\left|p_{0}(x)\right| \square_{0} \varepsilon_{0} \wedge \bigwedge_{i=1}^{n} \varepsilon_{i} \square_{i}\left|p_{i}(x)\right|
$$

be a good $K$-annulus formula and let $f \in \mathcal{O}_{K}^{\dagger}(\varphi)$. Then, if $f^{\sigma} \neq 0$, there exist a monic polynomial $P(x)$ with zeros only in $\mathcal{U}_{\varphi}$, integers $n_{i}$ and a strong unit $E \in \mathcal{O}_{K}^{\dagger}(\varphi)$ such that

$$
f^{\sigma}=P(x) \cdot \prod_{i=1}^{n} p_{i}(x)^{n_{i}} \cdot E^{\sigma} .
$$

$P, E$ and the $n_{i}$ are uniquely determined by $f($ and $\varphi)$.

Proof. We have already observed that $P \in K[x]$. We have to show that

$$
\prod_{i, j, k}\left(x-\alpha_{i j k}\right)^{n_{i j k}} \cdot E^{\sigma}
$$

can be written in the form

$$
\prod_{i=1}^{n} p_{i}(x)^{n_{i}} \cdot\left(E^{\prime}\right)^{\sigma} .
$$

Hence, we need to see, in the notation of (5.2), that $n_{i j k}$ is a multiple of the number of zeros that $p_{i}$ has in each hole in each $\mathcal{U}_{j}$. But this follows by comparing the representation (5.2) with the representations [5.1] that come (via Lemma 5.3.7) from the thin annuli 
surrounding the holes as in Definition 5.5.1. Finally, observe that if $p_{i}$ has $\ell$ zeros $\alpha=$ $\beta_{1}, \ldots, \beta_{\ell}$ in a hole, then $(x-\alpha)^{\ell}=\prod_{k=1}^{\ell}\left(x-\beta_{k}\right) \cdot E^{\prime}$ for some strong unit $E^{\prime}$.

Uniqueness of the representation (5.3) follows from the observations that $P(x)$ is determined by the zeros of $f$ and that $\prod_{i=1}^{n} p_{i}(x)^{n_{i}}$ is a strong unit only when $n_{i}=0$ for all $i$.

The following theorem is what we need for model-theoretic applications.

Theorem 5.5.3. Let $K$ be a valued field with separated analytic $\mathcal{A}$-structure, and let $\mathcal{L}_{\mathcal{A}(K)}$ be the language of valued fields, $\left\langle 0,1,+, \cdot,(\cdot)^{-1},|\cdot|\right\rangle$, augmented with function symbols for all the elements of $\bigcup_{m, n} A_{m, n}(K)$. (We extend functions $f \in A_{m, n}(K)$ by zero outside $\left(K^{\circ}\right)^{m} \times\left(K^{\circ \circ}\right)^{n}$.) Let x be one variable, and let $\tau(x)$ be a term of $\mathcal{L}_{\mathcal{A}(K)}$. There is a finite set $S \subset K_{\mathrm{alg}}^{\circ}$ and a finite cover of $K_{\mathrm{alg}}^{\circ}$ by $K$-annuli $\mathcal{U}_{i}$ such that for each $i$ there is a rational function $R_{i} \in K(x)$ and a strong unit $E_{i} \in \mathcal{O}_{K}^{\dagger}\left(\mathcal{U}_{i}\right)$ with

$$
\tau{\mid \mathcal{U}_{i} \backslash S}=R_{i} \cdot E_{i}^{\sigma} \mid \mathcal{U}_{i} \backslash S,
$$

i.e. $\tau$ and $R \cdot E_{i}$ define the same function on $\mathcal{U}_{i} \backslash S$. Observe that $K_{\mathrm{alg}}$ also has analytic $\mathcal{A}(K)$-structure (Theorem 4.5.11), $\tau$ is also a term of $\mathcal{L}_{\mathcal{A}\left(K_{\mathrm{alg}}\right)}$ and hence defines a function $K_{\text {alg }}^{\circ} \rightarrow K_{\text {alg. }}$.

Proof. This is proved by induction on terms (cf. [CLR1, Theorem 5.1]). First consider

$$
\tau(x)=f\left(\tau_{1}(x), \ldots, \tau_{m+n}(x)\right),
$$

where $f \in A_{m, n}(K)$. Then, by induction, we may assume that we have restricted to a $K$-annulus $\mathcal{U}$, and that there are rational functions $R_{j}$ and strong units $E_{j} \in \mathcal{O}_{K}^{\dagger}(\mathcal{U})$ such that for each $j$,

$$
\tau_{j}\left|\mathcal{U}=R_{j} \cdot E_{j}^{\sigma}\right| \mathcal{U}
$$

(We ignore the finite set $S$.) By Lemma 5.1.9 we can cover $\mathcal{U}$ with finitely many $K$ annuli $\mathcal{U}_{i}^{\prime}$ such that on each $\mathcal{U}_{i}^{\prime}$ for each $1 \leq j \leq m,\left|R_{j}(x) E_{j}(x)\right| \leq 1$ for all $x \in \mathcal{U}_{i}^{\prime}$ or $\left|R_{j}(x) E_{j}(x)\right|>1$ for all $x \in \mathcal{U}_{i}^{\prime}$; and for each $m+1 \leq i \leq m+n,\left|R_{j}(x) E_{j}(x)\right|<1$ for all $x \in \mathcal{U}_{i}^{\prime}$ or $\left|R_{j}(x) E_{j}(x)\right| \geq 1$ for all $x \in \mathcal{U}_{i}^{\prime}$. Then on each $\mathcal{U}_{i}^{\prime}$ there is an $f_{j}^{\prime} \in \mathcal{O}_{K}^{\dagger}\left(\mathcal{U}_{i}^{\prime}\right)$ that defines the same function as $\tau$, and the result follows from Theorem 5.5.2 and induction.

Next consider $\tau(x)=\tau_{1}(x) / \tau_{2}(x)$. As above we may assume that on $\mathcal{U}$ we have $\left.\tau_{i}\right|_{\mathcal{U} \backslash S}=\left.R_{i} \cdot E_{i}\right|_{\mathcal{U} \backslash S}$ for $i=1$, 2. Then $\left.\tau(x)\right|_{\mathcal{U} \backslash S}=R_{1} \cdot R_{2}^{-1} \cdot E_{1} \cdot E_{2}^{-1}$.

The case $\tau(x)=\tau_{1}(x) \cdot \tau_{2}(x)$ is similar.

Finally consider $\tau(x)=\tau_{1}(x)+\tau_{2}(x)$ on an annulus $\mathcal{U}$. Breaking into subannuli it is sufficient to consider the case that $\left|\tau_{1}(x)\right| \geq\left|\tau_{2}(x)\right|$ for all $x \in \mathcal{U}$. Write $\tau=\tau_{1}(1+$ $\left.\tau_{2} / \tau_{1}\right)$. Since $\left|\tau_{1}(x)\right| \geq\left|\tau_{2}(x)\right|$ for all $x \in \mathcal{U}$, it follows from Theorem 5.5.2 that if $\tau_{1}$ is not identically zero, then $\tau_{2} / \tau_{1} \in \mathcal{O}(\mathcal{U})$. Hence, also $1+\tau_{2} / \tau_{1} \in \mathcal{O}(\mathcal{U})$, and the result follows from the previous case.

Remark 5.5.4. With the notation from Remark 4.5.8, Theorem 5.5.3 also holds for $\mathcal{A}\left(K^{\prime}\right)$-terms, in which case the rational functions can be taken over $K^{\prime}$ and the annuli are $K^{\prime}$-annuli. 
Remark 5.5.5. The following statement (a Piecewise Mittag-Leffler Theorem) which is weaker than Theorem 5.5.2 suffices for the application in Theorem 5.5.3.

Let $\mathcal{U}$ be a $K$-annulus, and $f \in \mathcal{O}_{K}^{\dagger}(\mathcal{U})$. There is a finite cover of $\mathcal{U}$ by (thin and Laurent) $K$-annuli and open $K$-discs $\mathcal{U}_{i}$ such that for each $i$ there are polynomials $P_{i} \in K[x]$, integers $n_{i j}$ and strong units $E_{i} \in \mathcal{O}_{K}^{\dagger}\left(\mathcal{U}_{i}\right)$ such that

$$
f^{\sigma} \mid \mathcal{U}_{i}=P_{i} \prod_{j=1}^{n_{i}} p_{i j}^{n_{i j}} E_{i}^{\sigma}
$$

where the $p_{i j}$ are the polynomials occurring in a good description of $\mathcal{U}_{i}$.

The proof is easier than that of the full Mittag-Leffler Theorem (Theorem 5.5.2). It is easy to see that there is a cover (not necessarily rigid) of $\mathcal{U}$ by thin and Laurent annuli and discs. The thin annuli are handled by Lemma 5.3.7 Linear Laurent annuli can be handled using the canonical representation

$$
f(x)=\sum_{i} a_{i}\left(\frac{x-\alpha}{\varepsilon_{0}}-\frac{\varepsilon_{1}}{x-\alpha}\right)^{i}+\left(\frac{x-\alpha}{\varepsilon_{0}}\right) \sum_{i} b_{i}\left(\frac{x-\alpha}{\varepsilon_{0}}-\frac{\varepsilon_{1}}{x-\alpha}\right)^{i}
$$

with the $a_{i}, b_{i}$ in $K^{\circ}$. The result for Laurent $K$-annuli then follows as in the proof of Theorem 5.5.2 by comparing representations on thin $K$-annuli surrounding the holes. Discs are handled in Lemma 5.3.6.

\subsection{Strong Weierstrass systems}

By imposing extra axioms on the Weierstrass system, we can obtain stronger results than those of the previous section, and also prove some of the results of the previous section more easily and in a more elementary way. We do not need these results for the modeltheoretic applications in Section 6, but we present them here for completeness. Most of the examples of Weierstrass systems given in 4.4 satisfy these additional axioms.

Definition 5.6.1. We call a separated Weierstrass system $\left\{A_{m, n}\right\}$ a strong separated Weierstrass system if it satisfies the following five conditions:

(i) If $f\left(\xi, \eta_{1}, \eta_{2}, \rho\right) \in A_{m+2, n}$, there are $f_{1}\left(\xi, \eta_{1}, \zeta_{1}, \zeta_{2}, \rho\right), f_{2}\left(\xi, \eta_{2}, \zeta_{1}, \zeta_{2}, \rho\right)$ and $Q\left(\xi, \eta_{1}, \eta_{2}, \zeta_{1}, \zeta_{2}, \rho\right)$ in $A_{m+4, n}$ such that

$f\left(\xi, \eta_{1}, \eta_{2}, \rho\right)=f_{1}\left(\xi, \eta_{1}, \zeta_{1}, \zeta_{2}, \rho\right)+f_{2}\left(\xi, \eta_{2}, \zeta_{1}, \zeta_{2}, \rho\right)+Q \cdot\left(\eta_{1} \eta_{2}-\zeta_{1} \eta_{1}-\zeta_{2} \eta_{2}\right)$.

(ii) If $f\left(\xi, \rho, \lambda_{1}, \lambda_{2}\right) \in A_{m, n+2}$, there are $f_{1}\left(\xi, \rho, \lambda_{1}, \tau_{1}, \tau_{2}\right), f_{2}\left(\xi, \rho, \lambda_{2}, \tau_{1}, \tau_{2}\right)$ and $Q\left(\xi, \rho, \lambda_{1}, \lambda_{2}, \tau_{1}, \tau_{2}\right)$ in $A_{m, n+4}$ such that

$f\left(\xi, \rho, \lambda_{1}, \lambda_{2}\right)=f_{1}\left(\xi, \rho, \lambda_{1}, \tau_{1}, \tau_{2}\right)+f_{2}\left(\xi, \rho, \lambda_{2}, \tau_{1}, \tau_{2}\right)+Q \cdot\left(\lambda_{1} \lambda_{2}-\tau_{1} \lambda_{1}-\tau_{2} \lambda_{2}\right)$.

(iii) If $f\left(\xi, \eta_{1}, \rho, \lambda_{1}\right) \in A_{m+1, n+1}$, there are $f_{1}\left(\xi, \eta_{1}, \zeta_{1}, \rho, \tau_{1}\right), f_{2}\left(\xi, \zeta_{1}, \rho, \lambda_{1}, \tau_{1}\right)$ and $Q\left(\xi, \eta_{1}, \zeta_{1}, \rho, \lambda_{1}, \tau_{1}\right)$ in $A_{m+2, n+2}$ such that

$f\left(\xi, \eta_{1}, \rho, \lambda_{1}\right)=f_{1}\left(\xi, \eta_{1}, \zeta_{1}, \rho, \tau_{1}\right)+f_{2}\left(\xi, \zeta_{1}, \rho, \lambda_{1}, \tau_{1}\right)+Q \cdot\left(\eta_{1} \lambda_{1}-\tau_{1} \eta_{1}-\zeta_{1} \lambda_{1}\right)$. 
(iv) If $f\left(\xi, \eta_{1}, \eta_{2}, \rho\right) \in A_{m+2, n}$, there are $f_{1}\left(\xi, \eta_{1}, \eta_{3}, \rho\right), f_{2}\left(\xi, \eta_{2}, \eta_{3}, \rho\right)$ and $Q\left(\xi, \eta_{1}, \eta_{2}, \eta_{3}, \rho\right)$ in $A_{m+3, n}$ such that

$$
f\left(\xi, \eta_{1}, \eta_{2}, \rho\right)=f_{1}\left(\xi, \eta_{1}, \eta_{3}, \rho\right)+\eta_{2} f_{2}\left(\xi, \eta_{2}, \eta_{3}, \rho\right)+Q \cdot\left(\eta_{1} \eta_{2}-\eta_{3}\right) .
$$

(v) If $f\left(\xi, \rho, \lambda_{1}, \lambda_{2}\right) \in A_{m, n+2}$, there are $f_{1}\left(\xi, \rho, \lambda_{1}, \lambda_{3}\right), f_{2}\left(\xi, \rho, \lambda_{2}, \lambda_{3}\right)$ and $Q\left(\xi, \rho, \lambda_{1}, \lambda_{2}, \lambda_{3}\right)$ in $A_{m, n+3}$ such that

$$
f\left(\xi, \rho, \lambda_{1}, \lambda_{2}\right)=f_{1}\left(\xi, \rho, \lambda_{1}, \lambda_{3}\right)+\lambda_{2} f_{2}\left(\xi, \rho, \lambda_{2}, \lambda_{3}\right)+Q \cdot\left(\lambda_{1} \lambda_{2}-\lambda_{3}\right) .
$$

We call a strictly convergent Weierstrass system $\left\{A_{m}\right\}$ a strong strictly convergent Weierstrass system if it has properties (i) and (iv) with $n=0$.

Remark 5.6.2. The conditions of Definition 5.6.1 ensure that we can perform the formal operations on the power series in $A_{m, n}$ that are needed in this subsection. For example, parts (iv) and (v) allow us to write $f\left(X, X^{-1}\right)=f_{1}(X)+f_{2}\left(X^{-1}\right)$. Other parts will be used (suppressing extraneous variables) to allow us to use a relation of the form $X Y=$ $a X+b Y$, where $a$ and $b$ are constants, to write $f(X, Y)=f_{1}(X)+f_{2}(Y)$. This is what we need for partial fractions expansions. These conditions are not automatically satisfied for Weierstrass systems (see Example 4.4 (9)), though they are satisfied by most of the natural examples (and are a consequence of completeness).

For the rest of this subsection we assume that the field $K$ has strong separated analytic $\mathcal{A}$-structure, and hence strong separated analytic $\mathcal{A}(K)$-structure, coming from a strong separated Weierstrass system $\mathcal{A}=\left\{A_{m, n}\right\}$.

We will first reprove the basic Mittag-Leffler decomposition for linear annuli (Theorem 5.6.3. We give this proof in the "strong" case as it is much simpler than the proof given above when $\mathcal{A}$ is not necessarily strong.

Let $\varphi$ be a linear $K$-annulus formula, and let $f \in \mathcal{O}_{K}^{\dagger}(\varphi)$. Then

$$
f=\sum_{\nu} a_{v}(x)\left(\frac{x-\alpha_{0}}{\varepsilon_{0}}\right)^{\nu_{0}}\left(\frac{\varepsilon_{1}}{x-\alpha_{1}}\right)^{\nu_{1}} \cdots\left(\frac{\varepsilon_{n}}{x-\alpha_{n}}\right)^{\nu_{n}} .
$$

(Recall the slight abuse of notation about $\varepsilon$, discussed above Lemma 5.1.2) Doing Weierstrass Division by $x-\alpha_{0}-\varepsilon_{0}\left(\frac{x-\alpha_{0}}{\varepsilon_{0}}\right)$ we may assume that the $a_{v}$ are constants (i.e. of degree 0$)$. Next we observe that we can write

$$
f=f_{0}\left(x-\alpha_{0}\right)+\hat{f}\left(\frac{\varepsilon_{1}}{x-\alpha_{1}}, \ldots, \frac{\varepsilon_{n}}{x-\alpha_{n}}\right) .
$$

To see this, notice that by Weierstrass Division by $x-\alpha_{0}-\varepsilon_{0}\left[\frac{x-\alpha_{1}}{\varepsilon_{0}}+\frac{\alpha_{1}-\alpha_{0}}{\varepsilon_{0}}\right]$ we can write

$$
f=g\left(\frac{x-\alpha_{1}}{\varepsilon_{0}}, \frac{\varepsilon_{1}}{x-\alpha_{1}}, \ldots, \frac{\varepsilon_{n}}{x-\alpha_{n}}\right)
$$

and using Definition 5.6.1 (iv) (see Remark 5.6.2, we can write $g$ as

$$
g_{1}\left(\frac{x-\alpha_{1}}{\varepsilon_{0}}, \frac{\varepsilon_{2}}{x-\alpha_{2}}, \ldots, \frac{\varepsilon_{n}}{x-\alpha_{n}}\right)+\frac{\varepsilon_{1}}{x-\alpha_{1}} g_{2}\left(\frac{\varepsilon_{1}}{x-\alpha_{1}}, \ldots, \frac{\varepsilon_{n}}{x-\alpha_{n}}\right),
$$


and we may proceed by induction. Finally we use the observation that for $i \neq j$,

$$
\frac{\varepsilon_{i}}{x-\alpha_{i}} \frac{\varepsilon_{j}}{x-\alpha_{j}}=\frac{A}{x-\varepsilon_{i}}+\frac{B}{x-\varepsilon_{j}}
$$

where $A=\frac{\varepsilon_{1} \varepsilon_{2}}{\alpha_{1}-\alpha_{2}}$ and $B=\frac{\varepsilon_{1} \varepsilon_{2}}{\alpha_{2}-\alpha_{1}}$. We have $\left|\alpha_{1}-\alpha_{2}\right| \geq\left|\varepsilon_{1}\right|,\left|\varepsilon_{2}\right|$, and if at least one of the holes is "open" (i.e. defined by a strict inequality) then $\left|\alpha_{1}-\alpha_{2}\right|>\left|\varepsilon_{1}\right|,\left|\varepsilon_{2}\right|$. Now Definition 5.6.1 (i)-(iii) (see Remark 5.6.2) allow us to carry out the partial fractions expansion term by term to write

$$
f=f_{0}\left(\frac{x-\alpha_{0}}{\varepsilon_{0}}\right)+f_{1}\left(\frac{\varepsilon_{1}}{x-\alpha_{1}}\right)+\cdots+f_{n}\left(\frac{\varepsilon_{n}}{x-\alpha_{n}}\right) .
$$

By convention we put the constant term in $f_{0}$, so $f_{1}(\infty)=\cdots=f_{n}(\infty)=0$. The uniqueness of this expansion is then proved exactly as in [FP, Proposition 2.2.6]. This establishes the first part of the following

Theorem 5.6.3 (Mittag-Leffler Theorem for linear annuli). Let $\varphi$ be a linear $K$-annulus formula, and let $f \in \mathcal{O}_{K}^{\dagger}(\varphi)$.

(i) There is a unique $f_{0} \in \mathcal{O}_{K}^{\dagger}\left(\left|x-\alpha_{0}\right| \square_{0} \varepsilon_{0}\right)$ and unique $f_{i} \in \mathcal{O}_{K}^{\dagger}\left(\varepsilon_{i} \square_{i}\left|x-\alpha_{i}\right|\right)$ without constant terms such that

$$
f=f_{0}+\cdots+f_{n} .
$$

(ii) If $f \neq 0$ there is a unique rational function $R$ of the form $P(x) \prod_{i}\left(x-\alpha_{i}\right)^{n_{i}}$, where $P(x)$ is a monic polynomial all of whose zeros are in $\mathcal{U}_{\varphi}$ and the $n_{i}$ are integers, and a unique strong unit $E \in \mathcal{O}_{K}^{\dagger}(\varphi)$ such that

$$
f=R \cdot E .
$$

Proof (cf. [DHM]). We must prove part (ii). By part (i) we may assume that

$$
f=f_{0}\left(\frac{x-\alpha_{0}}{\varepsilon_{0}}\right)+\sum_{i=1}^{n} f_{i}\left(\frac{\varepsilon_{i}}{x-\alpha_{i}}\right)
$$

or more precisely that

$$
f \equiv f_{0}\left(z_{0}\right)+\sum_{i=1}^{n} f_{i}\left(z_{i}\right) \bmod \left(x-\alpha_{0}-\varepsilon_{0} z_{0}, \ldots, z_{n}\left(x-\alpha_{n}\right)-\varepsilon_{n}\right)
$$

where each $z_{i}$ is either a $\xi$ or a $\rho$ variable, and

$$
f_{0}\left(z_{0}\right)=\sum_{j=0}^{\infty} a_{0 j} z_{0}^{j}, \quad f_{i}\left(z_{i}\right)=\sum_{j=1}^{n} a_{i j} z_{i}^{j} .
$$

First we prove existence. 
We proceed by induction on $n$, the number of nonzero $f_{i}, i \geq 1$. If $n=0$, applying the Strong Noetherian Property and multiplying by a constant, we may assume that $f_{0}$ is regular. Hence, after multiplying by a strong unit we may assume that $f_{0}$ is a polynomial. If $n>0$, by using the Strong Noetherian Property and after multiplying by a constant, we may assume that $f$ is regular in $z_{i}$ of degree $N$ say for some $i \geq 0$. If $i=0$, we may assume after multiplying by a strong unit that $f_{0}$ is a polynomial. Rewrite $f_{0}$ as a polynomial in $\frac{x-\alpha_{1}}{\varepsilon_{1}}$ and multiply by $\left(\frac{x-\alpha_{1}}{\varepsilon_{1}}\right)^{-N}$ to reduce to the case that $f_{0}$ is 0 . Hence we need only consider the case $i>0$. Apply Weierstrass Preparation to multiply by a strong unit and reduce to the case that $f$ is a polynomial of degree $N$, say in $\frac{\varepsilon_{i}}{x-\alpha_{i}}$. Multiplying by $\left(\frac{x-\alpha_{i}}{\varepsilon_{i}}\right)^{N}$ (or $z_{i}^{-N}$ ) and using part (i) reduces $n$. Hence $f$ can be written in the form

$$
f=P(x) \cdot \prod_{i=1}^{n}\left(x-\alpha_{i}\right)^{n_{i}} \cdot E
$$

where $E$ is a strong unit.

Next we show that if $P(x)$ is irreducible and has no zeros in the disc $\left\{x:\left|x-\alpha_{0}\right| \square_{0} \varepsilon_{0}\right\}$ then $P(x)$ is a strong unit. We may make a change of variable so that $\alpha_{0}=0$ and $\varepsilon_{0}=1$. We may assume that $P(x)=a_{m} x^{m}+a_{m-1} x^{m-1}+\cdots+a_{0}$. If $\square_{0}$ is $\leq$ then all the zeros of $P(x)$ are $>1$ and hence $\left|a_{0}\right|>\left|a_{i}\right|$ for $i=1, \ldots, m$. Thus $a_{0}^{-1} \bar{P}(x)$ is a very strong unit (Definition 5.1.4), as it is regular of degree 0 . If $\square_{0}$ is $<$ then all the zeros of $P(x)$ are $\geq 1$, and since $|x|<1$ (i.e. is a $\rho$ variable) we again see that $a_{0}^{-1} P(x)$ is a very strong unit. Next we observe (cf. Proposition 5.3.4) that if $P$ is irreducible and has one of (and hence all of) its zeros in the hole $\left\{x:\left|x-\alpha_{i}\right| \bar{\square}_{i} \varepsilon_{i}\right\}$, and $P$ has degree $\ell$, then $P \cdot\left(x-\alpha_{i}\right)^{-\ell}$ is a strong unit. Finally, to prove uniqueness we must see that the only time that an expression of the form $P(x) \cdot \prod_{i=1}^{n}\left(x-\alpha_{i}\right)^{n_{i}}$ with all zeros of $P$ lying in $\mathcal{U}$ is a strong unit is when $P(x)$ is a constant and $n_{i}=0$ for all $i$. We may assume that $\alpha_{0}=0, \varepsilon_{0}=1$. Again considering the two cases that $\square_{0}$ is $\leq$ and $\square_{0}$ is $<$ separately, this is clear.

The following is an immediate consequence of the Mittag-Leffler Theorem for linear annuli, Theorem 5.6 .3

Corollary 5.6.4. If $\mathcal{U}$ is a linear $K$-annulus then $\mathcal{O}_{K}^{\dagger}(\mathcal{U})$ is a principal ideal domain and $\mathcal{O}_{K}^{\dagger}(\mathcal{U}) \simeq \mathcal{O}_{K}^{\sigma}(\mathcal{U})$.

Proof. Using the notation of Theorem 5.6.3, exactly as in [FP, Proposition 2.2.6], one has $\|f\|_{\text {sup }}=\max _{i}\left\|f_{i}\right\|_{\text {sup }}$, and $\left\|f_{i}\right\|=\left\|f_{i}\right\|_{\text {sup }}$.

The full Mittag-Leffler Theorem in the strong case is:

Theorem 5.6.5. If $\varphi$ is a good $K$-annulus formula and $f \in \mathcal{O}_{K}^{\dagger}(\varphi)$, then:

(i) there are unique

$$
\begin{aligned}
f_{0} & =\sum_{j=0}^{\infty} a_{0 j}(x)\left(\frac{p_{0}(x)}{\varepsilon_{0}}\right)^{j} \quad \text { with } \operatorname{deg} a_{0 j}<\operatorname{deg} p_{0} \\
f_{i} & =\sum_{j=1}^{\infty} a_{i j}(x)\left(\frac{\varepsilon_{i}}{p_{i}(x)}\right)^{j} \quad \text { with } \operatorname{deg} a_{i j}<\operatorname{deg} p_{i}
\end{aligned}
$$


such that

$$
f=f_{0}+f_{1}+\cdots+f_{n} .
$$

(This type of decomposition can fail to exist if the Weierstrass system is not strong, for example, in the structure described in 4.4(9) above.)

(ii) There is a unique representation of the form

$$
f(x)=P(x) \cdot \prod_{i} p_{i}(x)^{n_{i}} \cdot E
$$

where $P(x)$ is a monic polynomial all of whose zeros are in $\mathcal{U}_{\varphi}$, the $n_{i}$ are integers, and $E \in \mathcal{O}_{K}^{\dagger}(\varphi)$ is a strong unit.

Proof. As observed in Corollary 5.6.4, from the representation in (i) it follows easily that the mapping $f \mapsto f^{\sigma}$ is injective. Then (ii) follows from the linear case (Theorem 5.6.3) exactly as in the proof Theorem 5.5.2 by considering thin or Laurent annuli surrounding the holes. Recall (Remark 5.3.9) that the proof of Lemma 5.3.7 is much easier in the strong case.

We outline the proof of (i). If $\mathcal{H}_{i}$ is a hole of the form $\left|p_{i}(x)\right| \leq \varepsilon_{i}$ in $\mathcal{U}_{K}(\varphi)$, then considering $f$ on a Laurent annulus $\mathcal{U}_{i}=\left\{x \in K_{\text {alg }}: \varepsilon_{i}<\left|p_{i}(x)\right|<\delta_{i}\right\}$ surrounding this hole gives

$$
\left.f\right|_{\mathcal{U}_{i}}=\sum b_{j k}(x)\left(\frac{\varepsilon_{i}}{p_{i}(x)}\right)^{j}\left(\frac{p_{i}(x)}{\delta_{i}}\right)^{k}
$$

with $\operatorname{deg} b_{j k}(x)<\operatorname{deg} p_{i}(x)$, which, using Definition 5.6.1 and the relation $\frac{\varepsilon_{i}}{p_{i}} \frac{p_{i}}{\delta_{i}}=\frac{\varepsilon_{i}}{\delta_{i}}$, can be rewritten as

$$
\left.f\right|_{\mathcal{U}_{i}}=\sum_{j \geq 1} a_{i j}(x)\left(\frac{\varepsilon_{i}}{p_{i}(x)}\right)^{j}+\sum_{j \geq 0} a_{i j}^{\prime}(x)\left(\frac{p_{i}(x)}{\delta_{i}}\right)^{j} .
$$

Take $f_{i}:=\sum a_{i j}(x)\left(\varepsilon_{i} / p_{i}(x)\right)^{j}$.

If $\mathcal{H}_{i}$ is a hole of the form $\left|p_{i}(x)\right|<\varepsilon_{i}$ in $\mathcal{U}_{K}(\varphi)$, consider a thin $K$-annulus $\mathcal{U}_{i}$ surrounding $\mathcal{H}_{i}$. On $\mathcal{U}_{i}, f$ has a representation

$$
\left.f\right|_{\mathcal{U}_{i}}=\sum a_{i j k v}(x)\left(\frac{p_{i}(x)}{\varepsilon_{i}}\right)^{j}\left(\frac{\varepsilon_{i}}{p_{i}(x)}\right)^{k}\left(\frac{\varepsilon^{\prime}}{p^{\prime}(x)}\right)^{v} .
$$

Use a relation of the form

$$
\frac{\varepsilon_{i}}{p_{i}(x)} \frac{\varepsilon_{j}}{p_{j}(x)}=A(x) \frac{\varepsilon_{i}}{p_{i}(x)}+B(x) \frac{\varepsilon_{j}}{p_{j}(x)}
$$

where $\operatorname{deg} B<\operatorname{deg} p_{j}, \operatorname{deg} A<\operatorname{deg} p_{i}$ and $A$ and $B$ have supremum norm $\leq 1$ on $\mathcal{U}_{i}$, to separate the terms $\varepsilon_{i} / p_{i}(x)$ from $\varepsilon_{j} / p_{j}(x)$. Use the relation $\left(\frac{\varepsilon_{i}}{p_{i}(x)}\right)\left(\frac{p_{i}(x)}{\varepsilon_{i}}\right)=1$ to separate the terms $\varepsilon_{i} / p_{i}(x)$ and $p_{i}(x) / \varepsilon_{i}$. Thus $f$ can be written

$$
f=f_{i}+g
$$


where $f_{i}=\sum a_{i j}(x)\left(\varepsilon_{i} / p_{i}(x)\right)^{j}, \operatorname{deg} a_{i j}<\operatorname{deg} p_{i}$, and $g=\sum a_{k v^{\prime}}(x)\left(\frac{p_{i}(x)}{\varepsilon_{i}}\right)^{k}\left(\frac{\varepsilon^{\prime}}{p^{\prime}(x)}\right)^{v^{\prime}}$, where $v^{\prime}=\left(v_{1}, \ldots, v_{i-1}, v_{i+1}, \ldots, v_{n}\right)$. Use a thin or Laurent annulus at the "outer" edge of $\mathcal{U}$ to obtain $f_{0}$.

Finally, considering $f-\left(f_{0}+f_{1}+\cdots+f_{n}\right)$ on the linear $K_{\text {alg-annuli into which } \mathcal{U}}$ decomposes over $K_{\text {alg }}$, using Theorem 5.6.3 (i), we see that $\left\|f-\left(f_{0}+f_{1}+\cdots+f_{n}\right)\right\|_{\text {sup }}$ $=0$. The result now follows from Proposition 5.3.11 i).

Corollary 5.6.6. If $\mathcal{U}$ is a $K$-annulus then $\mathcal{O}_{K}^{\dagger}(\mathcal{U})$ is a principal ideal domain and $\mathcal{O}_{K}^{\dagger}(\mathcal{U}) \simeq \mathcal{O}_{K}^{\sigma}(\mathcal{U})$

\section{Cell decomposition}

In this section, a cell decomposition, as a consequence of $b$-minimality, is obtained for analytic structures on Henselian valued fields of Section 4 (see Remark 6.3.17). The history of cell decomposition for Henselian fields goes back to Cohen [Co] and Denef [D1], [D2] for the algebraic case, generalized by Pas [Pas1], [Pas2] to more general Henselian fields, and by the authors to analytic expansions [C], [CLR1], suited for $p$-adic integration. The definitions of cells and cell decomposition have been simplified in [CL1] and put in an axiomatic framework in [CL2]. In [CL1], cell decomposition is used to define motivic integrals; historically, cell decomposition has been used to calculate many types of $p$-adic integrals [D1], [Pas1]. In this section we generalize the cell decomposition of [CLR1] to the generality of this paper, using the axiomatic formulation of [CL2]. We also establish "preservation of balls" and the "Jacobian property" for these structures, notions useful for change of variables formulas for integrals. In this section we shall use the additively written order ord as well as the multiplicative norm $|\cdot|$.

\subsection{The semialgebraic language}

Let Hen be the collection of all Henselian valued fields of characteristic zero (hence mixed characteristic and equicharacteristic zero fields are included).

For $K$ in Hen, write $K^{\circ}$ for the valuation ring, $\Gamma_{K}$ for the value group, ord : $K^{\times} \rightarrow \Gamma_{K}$ for the (additively written) valuation, $M_{K}$ or $K^{\circ \circ}$ for the maximal ideal of $K^{\circ}$, and $\widetilde{K}$ for the residue field.

For any integer $n>0$, write

$$
\mathrm{rv}_{n}: K^{\times} \rightarrow K^{\times} / 1+n M_{K}
$$

for the natural group morphism, with $n M_{K}=\left\{n m: m \in M_{K}\right\}$, and extend it to a map $\operatorname{rv}_{n}: K \rightarrow\left(K^{\times} / 1+n M_{K}\right) \cup\{0\}$ by sending zero to zero. Write $R V_{n}$, or $R V_{n}(K)$, for $\left(K^{\times} / 1+n M_{K}\right) \cup\{0\}$ for integers $n>0$. Write also ord for the natural maps ord : $K^{\times} / 1+n M_{K} \rightarrow \Gamma_{K}$. We sometimes abbreviate $\mathrm{rv}_{1}$ to rv and $R V_{1}$ to $R V$. Note that in equicharacteristic zero the $R V_{n}$ all are the same as $R V_{1}$.

The sorts $R V$ and $R V_{n}$ are called auxiliary. The valued field sort is the main sort. There are no other sorts. We write Val for the valued field sort. 
Definition 6.1.1. Let $\mathcal{L}_{\text {Hen }}$ be the language of rings $(+,-, \cdot, 0,1)$ for the valued field sort, together with function symbols $\operatorname{rv}_{n}$ for all $n>0$, and the inclusion language as defined below on the auxiliary sorts.

Let $\mathcal{T}_{\text {Hen }}$ be the theory of all fields in Hen in the language $\mathcal{L}_{\text {Hen }}$.

We will sometimes write $K$ to denote both a model of $\mathcal{T}_{\text {Hen }}$ and the (universe of the) valued field sort of that model.

6.1.2. The inclusion language on the $R V_{n}$. Let $K$ be in Hen. For $a_{i} \in R V_{n_{i}}(K), i=$ $1, \ldots, n$, and for $f, g$ polynomials over $\mathbb{Z}$ in $n$ variables, we let the expression

$$
f\left(a_{1}, \ldots, a_{n}\right)
$$

correspond to the set

$$
f(a):=\left\{x \in K:\left(\exists y \in K^{n}\right)\left(f(y)=x \wedge \bigwedge_{i} \operatorname{rv}_{n_{i}}\left(y_{i}\right)=a_{i}\right)\right\} .
$$

By an inclusion

$$
f(a) \subset g(a)
$$

of such expressions, we shall mean the inclusion of the corresponding sets.

The inclusion language $\mathcal{L}_{R V}$ on the sorts $R V_{n}, n>0$, consists of the three symbols $+, \cdot, \subset$, interpreted as explained in 6.1 . There are no terms in this language, only relations of the form $f(x) \subset g(x)$, for $f$ and $g$ polynomials in $x_{1}, \ldots, x_{n}$ formed with + and ·, with the $x_{i}$ variables of the sorts $R V_{n_{i}}$ for some integers $n_{i}>0$.

Remark 6.1.3. (i) For an alternative (but essentially equivalent) language on the auxiliary sorts, see $[\overline{\mathrm{BK}}]$ and $[\mathrm{Scan} 1]$.

(ii) Note that $\operatorname{ord}(x)<\operatorname{ord}(y)$ is valued field quantifier free definable using rv, for example, by $\operatorname{rv}(x)=\operatorname{rv}(x+y)$.

Definition 6.1.4. By an open, resp. closed, ball in a valued field $K$ is meant a set of the form

$$
\{x \in K: \operatorname{ord}(x-b)>\operatorname{ord}(a)\}, \quad \text { resp. } \quad\{x \in K: \operatorname{ord}(x-b) \geq \operatorname{ord}(a)\},
$$

with $b \in K, a \in K^{\times}$. By a point is meant a singleton.

Remark 6.1.5. For any $n>0$, any nonzero $\xi \in R V_{n}$, and any $h \in K$, the set

$$
X:=\left\{x \in K: \operatorname{rv}_{n}(x-h)=\xi\right\}
$$

is an open ball of the form

$$
\{x \in K: \operatorname{ord}(x-b)>\alpha\}
$$

for any $b \in X$ and $\alpha=\operatorname{ord}(n(b-h))$. Often, none of the points $b$ is definable (over a certain set of parameters) while $h$ and $\xi$ are definable. This is the advantage of the description 6.2) of the open ball $X$. 
The following is a consequence of Hensel's Lemma (see also [Co], [D2], [Pas1], [Pas2]).

Lemma 6.1.6. Let $K$ be in Hen. Let

$$
f(y)=\sum_{i=0}^{m} a_{i} y^{i}
$$

be a polynomial in $y$ with coefficients in $K$, let $n>0$ be an integer, and let $x_{0} \neq 0$ be in $R V_{n}(K)$. Suppose that there exist $i_{0}>0$ and $x \in K$ with

$$
\operatorname{rv}_{n}(x)=x_{0} \quad \text { and } \quad \operatorname{ord}\left(a_{i_{0}} x^{i_{0}}\right) \text { is minimal among the } \operatorname{ord}\left(a_{i} x^{i}\right) \text {, }
$$

in the sense that

$$
\min _{0 \leq i \leq m} \operatorname{ord}\left(a_{i} x^{i}\right)=\operatorname{ord}\left(a_{i_{0}} x^{i_{0}}\right),
$$

and such that

$$
\operatorname{ord}(f(x))>\operatorname{ord}\left(n^{2} a_{i_{0}} x^{i_{0}}\right)
$$

and

$$
\operatorname{ord}\left(f^{\prime}(x)\right) \leq \operatorname{ord}\left(n a_{i_{0}} x^{i_{0}-1}\right) .
$$

Then there exists a unique $b \in K$ with

$$
f(b)=0 \quad \text { and } \quad \operatorname{rv}_{n}(b)=x_{0} .
$$

Proof. The case that the $a_{i}$ lie in the valuation ring $K^{\circ}$ and that $a_{i_{0}}$ and $x$ are units in $K^{\circ}$ follows from Hensel's lemma. The general case follows after changing coordinates. See [Pas1, Lemma 3.5] or [Pas2] for explicit change of variables.

Definition 6.1.7 (Henselian functions). Let $K$ be in Hen. For any integers $m \geq 0, n>0$, define the function

$$
h_{m, n}: K^{m+1} \times R V_{n}(K) \rightarrow K
$$

as the function sending the tuple $\left(a_{0}, \ldots, a_{m}, x_{0}\right)$ with nonzero $x_{0}$ to $b$ if there exist $i_{0}$ and $x$ that satisfy the conditions 6.3 6.5 of Lemma 6.1.6 and where $b$ is the unique element satisfying (6.6), and sending $\left(a_{0}, \ldots, a_{m}, x_{0}\right)$ to 0 in all other cases.

Define $\mathcal{L}_{\text {Hen }}^{*}$ as the union of the language $\mathcal{L}_{\text {Hen }}$ together with all the function symbols $h_{m, n}$. The functions $h_{m, n}$ are similar to those in [CLR1].

\subsection{The analytic languages}

Let $\mathcal{A}=\left\{A_{m, n}\right\}$ be a separated Weierstrass system, as defined in Section 4 . Define $\mathcal{L}_{\mathrm{Hen}, \mathcal{A}}$ as the language $\mathcal{L}_{\mathrm{Hen}}$ together with function symbols for all the elements of $\bigcup_{m, n} A_{m, n}$, with the field inverse $(\cdot)^{-1}$ on the valued field sort extended by $0^{-1}=0$, and together with the induced language on the sorts $R V_{n}$. The analytic function symbols are interpreted as zero outside their natural domains of products of the valuation ring and the maximal ideal. On their natural domains, they are interpreted via an analytic $\mathcal{A}$-structure. 
Let $\mathcal{T}_{\text {Hen, } \mathcal{A}}$ be the $\mathcal{L}_{\text {Hen, } \mathcal{A}}$-theory of all Henselian valued fields in Hen with analytic $\mathcal{A}$-structure.

Likewise, one can give definitions of analytic languages and analytic theories arising from strictly convergent Weierstrass systems and strictly convergent analytic structures with $\pi \neq 1$, as defined in Section 4.3 and Definition 4.3 .6 (i). We will not treat this separately.

\section{3. b-minimality}

To obtain cell decomposition, the criterion of $b$-minimality of [CL2] is used. From $b$ minimality many properties, like cell decomposition, dimension theory, etc., follow immediately (see [CL2]). In this paper we need only prove $b$-minimality, because cell decomposition follows by [CL2] (see Remark 6.3.17].

First we recall the definitions for $b$-minimality.

By an expansion of a theory $\mathcal{T}$ in a language $\mathcal{L}$ is meant a theory $\mathcal{T}^{\prime}$ in a language expanding $\mathcal{L}$ such that $\mathcal{T}^{\prime}$ contains $\mathcal{T}$. By definable is meant definable with parameters, unless we specify the parameters, for example by saying $A$-definable. A definable set is called auxiliary if it is a subset of a finite Cartesian product of (the universes of) auxiliary sorts.

Definition 6.3.1 $\left(b\right.$-minimality for expansions of $\left.\mathcal{T}_{\mathrm{Hen}}\right)$. Call an expansion of $\mathcal{T}_{\mathrm{Hen}} b$ minimal when the following three conditions are satisfied for every model $K$, any set of parameters $A$ (the elements of $A$ can belong to any of the sorts), for all $A$-definable subsets $X$ and $Y$ of (the valued field) $K$, and for every $A$-definable function $F: X \rightarrow Y$ :

(b1) there exists an $A$-definable function $f: X \rightarrow S$ with $S$ auxiliary such that for each $s \in f(X)$ the fiber $f^{-1}(s)$ is a point or an open ball;

(b2) if $g$ is a definable function from an auxiliary set to a ball, then $g$ is not surjective;

(b3) there exists an $A$-definable function $f: X \rightarrow S$ with $S$ auxiliary such that for every $s$ in $S$ the restriction $F_{\mid f^{-1}(s)}$ is either injective or constant.

Call $f$ as in (b1) a b-map on $X$. (The $f$ and $S$ of (b1) and (b3) are allowed to be different.)

Definition 6.3.2 (Centers for expansions of $\mathcal{T}_{\mathrm{Hen}}$ ). Say that a $b$-minimal expansion of $\mathcal{T}_{\text {Hen }}$ has centers when, in (b1) of Definition 6.3.1, one can choose $f$ such that moreover there exist $n>0$ and an $A$-definable function $h: f(X) \rightarrow K$ such that for each $s \in f(X)$ with $f^{-1}(s)$ a ball, there exists a (necessarily unique and nonzero) $\xi$ such that

$$
f^{-1}(s)=\left\{x \in K: \operatorname{rv}_{n}(x-h(s))=\xi\right\} .
$$

Following [CL2], call $h$ a $B_{n}$-center of $f$.

Remark 6.3.3. The definition of centers should be compared with Remark 6.1.5.

Definition 6.3.4 (Preservation of all balls for expansions of $\mathcal{T}_{\text {Hen }}$ ). Say that a $b$-minimal expansion of $\mathcal{T}_{\text {Hen }}$ preserves all balls when for every $F$ as in Definition 6.3.1, $f: X \rightarrow S$ can be taken as in (b3) such that moreover for each $s \in S$ and for each open ball $Z$ with $Z \subset f^{-1}(s), F(Z)$ is either an open ball or a point. 
A function on an open subset of a valued field is called $C^{1}$ when it is continuously differentiable in the sense of Cauchy's $\varepsilon, \delta$ definition.

Definition 6.3.5. Let $F: B_{1} \rightarrow B_{2}$ be a bijection between open balls $B_{1}, B_{2} \subset K$, with $K$ a Henselian valued field. Say that $F$ has the Jacobian property if the following conditions hold:

(a) $F$ is $C^{1}$ on $B_{1}$; write $\mathrm{Jac} F$ for $\partial F / \partial x: B_{1} \rightarrow K$;

(b) $\operatorname{rv}(\operatorname{Jac} F)$ is constant on $B_{1}$; write $\operatorname{rv}(\operatorname{Jac} F)=a$;

(c) for all $x, y \in B_{1}$ with $x \neq y$, one has

$$
\operatorname{ord}(a)+\operatorname{ord}(x-y)=\operatorname{ord}(F(x)-F(y)) .
$$

The Jacobian property of the following definition is useful for change of variables formulas, for example for $p$-adic or motivic integrals.

Definition 6.3.6. Say that an expansion $\mathcal{T}$ of $\mathcal{T}_{\text {Hen }}$ has the Jacobian property if the following holds in any model $K$ of $\mathcal{T}$ and any set $A$ of parameters:

For any $A$-definable bijection $F: X \subset K \rightarrow Y \subset K$ there exists a $A$-definable $b$-map $f: X \rightarrow S$ such that for each $s \in f(X)$ such that $f^{-1}(s)$ is a ball, the restriction of $F$ to $f^{-1}(s)$ has the Jacobian property.

The main results of this section are the following two theorems:

Theorem 6.3.7 ( $b$-minimality, $\operatorname{Char}(K)=0)$. Let $\mathcal{A}=\left\{A_{m, n}\right\}$ be a separated Weierstrass system, as defined in Section 4 . The theory $\mathcal{T}_{\mathrm{Hen}, \mathcal{A}}$ eliminates valued field quantifiers, is b-minimal with centers and preserves all balls. Moreover, $\mathcal{T}_{\mathrm{Hen}, \mathcal{A}}$ has the Jacobian property.

Define $\mathcal{L}_{\text {Hen, } \mathcal{A}}^{*}$ as the language $\mathcal{L}_{\text {Hen, } \mathcal{A}}$ together with all the functions $h_{m, n}$.

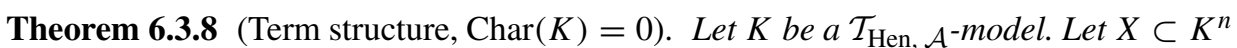
be definable and let $f: X \rightarrow K$ be an $\mathcal{L}_{\mathrm{Hen}, \mathcal{A}}(A)$-definable function for some set of parameters $A$. Then there exists an $\mathcal{L}_{\mathrm{Hen}, \mathcal{A}}(A)$-definable function $g: X \rightarrow S$ with $S$ auxiliary such that

$$
f(x)=t(x, g(x))
$$

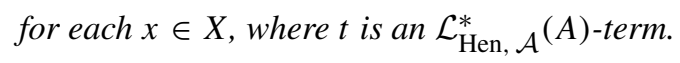

The rest of Section 6.3 is devoted to the proofs of these theorems. We begin with five lemmas, for $K$ in Hen. We will abuse notation and write $f$ for $f^{\sigma}$ when $f$ is in some $\mathcal{O}_{K}^{\dagger}(\cdot)$.

Lemma 6.3.9 $(\operatorname{Char}(K)=0)$. Let $B$ be the open ball $K_{\mathrm{alg}}^{\circ}$. Note that $B$ is a $K$-annulus. Let $g$ be in $\mathcal{O}_{K}^{\dagger}(B)$. Suppose that $\left|g^{\prime}(x)\right|=1$ for all $x \in K^{\circ \circ}$ and that $g(0)=0$. Then there exists an integer $n_{0}$ such that the mapping $x \mapsto g(x)$ is a bi-analytic isometry between $n_{0} K^{\circ \circ}$ and itself. 
Proof. Let $c \in K$ be such that $\left\|g^{\prime}\right\|=|1 / c|$. Since $c g^{\prime}$ lives in $A_{0,1}(K)$, it is regular of some degree, hence, by Weierstrass Preparation for $A_{0,1}(K), c g^{\prime}$ equals a monic polynomial $p$ over $K^{\circ}$ times a very strong unit $E$ in $A_{0,1}(K)$, for well chosen $c$. Suppose that $c$ is infinitesimal (in the sense that $|c|<|n|$ for all integers $n>0$ ). Let $K^{\prime}$ be the fraction field of the quotient of $K^{\circ}$ by the ideal $J$ consisting of the infinitesimals in $K^{\circ}$. By example (11) in Section 4.4, $K^{\prime}$ has analytic $\mathcal{A}(K) / J$-structure. Let $p_{J}$, resp. $E_{J}$, be the image of $p$, resp. $E$, in $A_{0,1} / J A_{0,1}$. Then $E_{J}$ is a very strong unit, hence has no zeros, and $p_{J}$ is a monic polynomial. Thus $p_{J} E_{J}$ is nonzero in $A_{0,1} / J A_{0,1}$ but it has infinitely many zeros in $K_{\mathrm{alg}}^{\prime}$, hence $p_{J}$ has infinitely many zeros in $K_{\mathrm{alg}}^{\prime}$, which is a contradiction. Hence, $c$ is not infinitesimal and there exists an integer $n_{0} \stackrel{\text { ag }}{>} 0$ such that $|c| \geq\left|n_{0}\right|$. Let $q$ be the image of $p$ in $\mathcal{O}_{K}\left(n_{0} K_{\mathrm{alg}}^{\circ}\right)$. Then $q$ is a strong unit of constant size $|q(x)| \geq\left|n_{0}\right|$ for $x \in n_{0} K_{\text {alg }}^{\circ}$, by definition of $\mathcal{O}_{K}\left(n_{0} K_{\mathrm{alg}}^{\circ}\right)$ and by Remark 4.1.7 Hence, if $\left|n_{0}\right|$ is small enough (we can always make $n_{0}$ more divisible if needed), then the image $h$ of $g$ in $\mathcal{O}_{K}\left(n_{0} K_{\mathrm{alg}}^{\circ \circ}\right)$ is regular of degree 1. (Indeed, automatically $h /\|h\|$ is regular of some degree $\alpha$, and then one can replace $n_{0}$ by $\alpha \cdot n_{0}$.) (Regularity of $h$ of degree 1 means that $h\left(n_{0} \rho\right)$ is regular of degree 1 in $A_{0,1}(K)$.) It then follows for such $n_{0}$ that $h: n_{0} K_{\mathrm{alg}}^{\circ \circ} \rightarrow n_{0} K_{\mathrm{alg}}^{\circ \circ}$ and also $h: n_{0} K^{\circ \circ} \rightarrow n_{0} K^{\circ \circ}$ are isometries.

Remark 6.3.10. Suppose that there is $n>0$ such that $\operatorname{rv}_{n}\left(g^{\prime}\right)=1$ on $B$. Then we can choose $n_{0}$ in Lemma 6.3.9 such that moreover

$$
\operatorname{rv}_{n}(x-y)=\operatorname{rv}_{n}(g(x)-g(y))
$$

for all $x \neq y$ in $n_{0} K^{\circ \circ}$. This is stronger than saying that $x \mapsto g(x)$ is an isometry between $n_{0} K^{\circ \circ}$ and itself. This property also follows from the regularity of degree 1 of $h$ at the end of the proof of Lemma 6.3.9

Lemma 6.3.11 $(\operatorname{Char}(K)=0)$. Fix elements $b_{i}$ in $K$ for $i=0, \ldots, m$, fix an integer $n>0$, and $x$ in $K$. Let $B$ be the Cartesian product

$$
\prod_{i=0}^{m}\left(b_{i}+n^{2} b_{i} K^{\circ \circ}\right)
$$

where $b_{i}+n^{2} b_{i} K^{\circ \circ}=\{0\}$ whenever $b_{i}=0$. Then the map

$$
F: B \rightarrow K: a \mapsto h_{m, n}\left(a, \operatorname{rv}_{n}(x)\right)
$$

is given by some $f$ in $\mathcal{O}^{\dagger}(B)$.

Proof. If $F$ is constantly zero on $B$ there is nothing to prove. In the other case, $F$ is nowhere zero on $B$, by the definition of $h_{m, n}$. Suppose thus that $F$ is nowhere zero on $B$. First suppose that the $b_{i}$ lie in $K^{\circ}$, that $b_{i_{0}}$ is a unit and that $\operatorname{ord}(x)=0$, where $i_{0}$ in $\{1, \ldots, m\}$ is such that ord $\left(b_{i_{0}}\right)$ is minimal among the ord $\left(b_{i}\right)$ in the sense that

$$
\min _{0 \leq i \leq m} \operatorname{ord}\left(b_{i}\right)=\operatorname{ord}\left(b_{i_{0}}\right) \text {. }
$$


Define

$$
g\left(\lambda_{1}, \rho_{0}, \ldots, \rho_{m}\right):=\sum_{0=1}^{m}\left(n^{2} b_{i} \rho_{i}+b_{i}\right)\left(n \lambda_{1}-x\right)^{i}
$$

in $A_{0, m+2}(K)$, where $\lambda_{1}$ runs over $K^{\circ \circ}$ and $\rho$ over $\left(K^{\circ \circ}\right)^{m+1}$. Since $b_{i_{0}}$ and $x$ are units, since $F$ is nonzero, by conditions (6.4) and (6.5), and by the definition of $h_{m, n}$, for all $\left(\lambda_{1}, \rho\right) \in\left(K_{\mathrm{alg}}^{\circ \circ}\right)^{m+2}$ one has

$$
\operatorname{ord}\left(g\left(\lambda_{1}, \rho\right)\right)>\operatorname{ord}\left(n^{2}\right) \quad \text { and } \operatorname{ord}\left(g^{\prime}\left(\lambda_{1}, \rho\right)\right) \leq \operatorname{ord}(n),
$$

with $g^{\prime}$ the derivative of $g$ with respect to $\lambda_{1}$. Rewrite

$$
g\left(\lambda_{1}, \rho\right)=\sum_{i=0}^{m} h_{i}(\rho) \lambda_{1}^{i} .
$$

Then, by Taylor's Theorem, $\left|h_{1}(\rho)\right| \geq|n|$ for all $\rho \in K_{\mathrm{alg}}^{\circ \circ}$, thus $h_{1}(\rho)^{-1}$ comes from an element of $A_{0, m+1}^{\dagger}(K)$ and $h_{1}(\rho)^{-1} \cdot g$ is regular of degree 1 in $\lambda_{1}$ in $A_{0, m+2}(K)$. Hence, by Weierstrass Preparation for $A_{0, m+2}(K)$ one has

$$
g=E\left(\lambda_{1}, \rho\right)\left(\lambda_{1}+h(\rho)\right)
$$

with $h(\rho)$ in $A_{0, m+1}(K), E$ a unit in $A_{0, m+2}(K)$, and with $\lambda_{1}+h(\rho)$ regular of degree 1 in $\lambda_{1}$. So, by Weierstrass Division one can compose $g$ and $-h$. Thus, in this case, one can take $-h(\rho)$ for $f$. In the general case, one can perform a change of variables as in the proof of Lemma 6.1.6 to reduce to the above special case, by using that the rings $\mathcal{O}^{\dagger}(\cdot)$ are closed under meaningful composition.

Lemma 6.3.12 $(\operatorname{Char}(K)=0)$. Let $n>0$ be an integer. Let $B$ be the open ball $K_{\mathrm{alg}}^{\circ \circ}$. Note that $B$ is a $K$-annulus. Let $E$ in $\mathcal{O}_{K}^{\dagger}(B)$ be a strong unit (cf. Definition 5.1.4). Then $\operatorname{rv}_{n}\left(E^{\sigma}\right)(x)$ only depends on $\operatorname{rv}_{n}(x)$ when $x$ varies over $K_{\mathrm{alg}}^{\circ}$.

Proof. The unit $E$ in $\mathcal{O}_{K}^{\dagger}(B)=A_{0,1}(K) \otimes_{K^{\circ}} K$ is automatically of the form

$$
E\left(\rho_{1}\right)=c+\rho_{1} g\left(\rho_{1}\right)
$$

for some $c \in K$ and $g \in \mathcal{O}_{K}^{\dagger}(B)$ such that $|c| \geq\|g\|$ (cf. Remark 4.1.7). Now suppose that $x_{1}, x_{2} \in K_{\mathrm{alg}}^{\circ \circ}$ with $\operatorname{rv}_{n}\left(x_{1}\right)=\operatorname{rv}_{n}\left(x_{2}\right)$. Then $x_{1}=(1+n a) x_{2}$ for some $a \in K_{\mathrm{alg}}^{\circ \circ}$. It is enough to treat the case $c=1$ (after dividing by $c$ ). Then

$$
\begin{aligned}
E\left(x_{1}\right) & =1+(1+n a) x_{2} g\left((1+n a) x_{2}\right)=1+(1+n a) x_{2} g\left(x_{2}\right)+n a h_{1} \\
& =1+x_{2} g\left(x_{2}\right)+n a h_{2}=\left(1+x_{2} g\left(x_{2}\right)\right)\left(1+n a h_{2}\left(1+x_{2} g\left(x_{2}\right)\right)^{-1}\right) \\
& =E\left(x_{2}\right)\left(1+n a h_{2}\left(1+x_{2} g\left(x_{2}\right)\right)^{-1}\right),
\end{aligned}
$$

with $h_{1}$ and $h_{2}$ in $K^{\circ}$, by Taylor expansion of $g\left((1+n a) x_{2}\right)$ around $x_{2}$. Now we are done since $n a h_{2}\left(1+x_{2} g\left(x_{2}\right)\right)^{-1}$ lies in $n K^{\circ \circ}$. 
Remark 6.3.13. The algebraic analogue of Lemma 6.3.14, namely, with $\mathcal{T}_{\text {Hen }}$ instead of $\mathcal{T}_{\text {Hen, } \mathcal{A}}$ and $\mathcal{L}_{\mathrm{Hen}}(A)$ instead of $\mathcal{L}_{\text {Hen, } \mathcal{A}}(A)$, follows from [CL2, Lemma 7.2.11 and Proposition 7.2.4], or from [CL2, Theorems 7.2.6 and 7.2.9].

Lemma 6.3.14 $(\operatorname{Char}(K)=0)$. Let $K$ be a $\mathcal{T}_{\mathrm{Hen}, \mathcal{A}}$-model, let $n>0$ be an integer, and let $f_{i}(y)$ be $\mathcal{L}_{\mathrm{Hen}, \mathcal{A}}(A)$-valued field terms in the $K$-variable y for $i=1, \ldots, k$, with $A a$ set of parameters. Then:

(i) There exists an $\mathcal{L}_{\mathrm{Hen}, \mathcal{A}}(A)$-definable b-map (cf. Definition 6.3.1) $\lambda: K \rightarrow S$ with $B_{n}$-center $c: \lambda(K) \rightarrow K$ such that for each $i, \mathrm{rv}_{n} \circ f_{i}$ is a component function of $\lambda$ (that is, $\lambda$ composed with a coordinate projection gives $\operatorname{rv}_{n}\left(f_{i}\right)$ ).

(ii) One can ensure that the image of $\lambda$ is defined by an $\mathcal{L}_{\mathrm{Hen}, \mathcal{A}}(A)$-formula without valued field quantifiers and that $c$ is given by an $\mathcal{L}_{\mathrm{Hen}, \mathcal{A}}^{\star}(A)$-term.

Proof. We may suppose that $A \subset K$, since $\mathcal{L}_{\text {Hen, } \mathcal{A}}(A)$-valued field terms cannot involve auxiliary constants. Let $K^{\prime}$ be the valued subfield of $K$ given by all $\mathcal{L}_{\text {Hen, } \mathcal{A}}(A)$-valued field terms. The field $K^{\prime}$ clearly has analytic $\mathcal{A}$-structure. Then $K$ has analytic $\mathcal{A}\left(K^{\prime}\right)$ structure by Remark 4.5.8, extending the $\mathcal{A}$-structure. Since the $\mathcal{T}_{\text {Hen, } \mathcal{A}}(A)$-valued field terms are the same as the $\mathcal{T}_{\text {Hen, }} \mathcal{A}\left(K^{\prime}\right)$-valued field terms, we may suppose that $\mathcal{A}\left(K^{\prime}\right)=\mathcal{A}$ and that $A$ is empty.

Apply Theorem 5.5.3 and Remark 5.5.4 to each of the $f_{i}$, yielding a finite number of polynomials over $K^{\prime}$, rational functions over $K^{\prime}$ and strong units. Write $p_{\ell}$ for the polynomials (including the denominators and numerators of the rational functions). Now by Remark 6.3.13 applied to the polynomials $p_{\ell}$, Remark 5.5.4 and by Lemma 6.3.12. and since on the auxiliary sorts lies the full induced language, there exist functions $\lambda$ and $c$ as desired.

The next lemma is only needed for the ball preservation property statement of Theorem 6.3.7, it is not needed to prove $b$-minimality and we use $b$-minimality once in the proof.

Lemma 6.3.15 $(\operatorname{Char}(K)=0)$. Let $t(x, u)$ be an $\mathcal{L}_{\mathrm{Hen}, \mathcal{A}}^{*}(A)$-valued field term in the valued field variable $x$, with $u$ a fixed tuple of elements of $A$ of auxiliary sorts. Let $n>0$ be an integer. Then there exists a b-map $f: K \rightarrow S$ for some auxiliary $S$ such that for each ball $B$ of the form $f^{-1}(s)$ the restriction of the function $x \mapsto t(x, u)$ to $B$ lies in $\mathcal{O}_{K}^{\dagger}(B)$.

Proof. We give a proof by induction on the complexity of the term $t$. Suppose that terms $t_{i}(x, u), i=0, \ldots, m$ with $m>0$, have a $b$-map $f_{i}: K \rightarrow S_{i}$ as in the lemma. Define $f$ as

$$
f: K \rightarrow \prod_{i} S_{i}: x \mapsto\left(f_{i}(x)\right)_{i}
$$

Then, since $\mathcal{O}_{K}^{\dagger}(B)$ is a ring for any ball $B$ and since the intersection of two open balls is either empty or an open ball, the lemma is satisfied for this $f$ when $t$ is the term $t_{1}+t_{2}$ and likewise for the product $t_{1} \cdot t_{2}$. Finally we treat the term $h_{m, n}\left(t_{0}, \ldots, t_{m}, u_{1}\right)$ for $n>0$. By $b$-minimality and a compactness argument (see, for example, the section on cell decomposition in [CL2]), we may suppose that the $\operatorname{rv}_{n^{2}}\left(t_{i}(x, u)\right)$ are constant on each 
fiber $f^{-1}(s)$. Apply Lemma 6.3.11 and the fact that the rings $\mathcal{O}(B)^{\dagger}(K)$ are closed under (meaningful) composition to see that this $f$ is as desired. The term $(\cdot)^{-1}$ is a special case of the functions $h_{m, n}$ (see the proof of Theorem 6.3.8) so the lemma is proved.

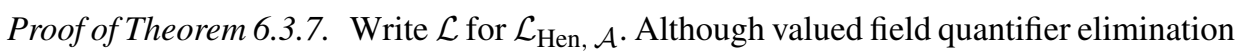
can be proven for $\mathcal{L}$ in the same way as it is historically done for many of the examples in Section 4, we give a slightly different proof. So, let us first prove elimination of valued field quantifiers. Let $K$ be a $\mathcal{T}_{\text {Hen, } \mathcal{A}}$-model. Let $\varphi(\xi, x, w)$ be a valued field quantifier free $\mathcal{L}$-formula with auxiliary variables $\xi$ running over say $S$, valued field variables $x$ running over $K^{m}$ and one valued field variable $w$. We have to prove that there exists a valued field quantifier free formula $\theta$ such that

$$
\{(\xi, x): K \models \exists w \varphi(\xi, x, w)\}=\{(\xi, x): K \models \theta(\xi, x)\} .
$$

Let $\tau_{i}$ be the valued field terms occurring in $\varphi$. By rewriting $\varphi$, we may suppose that these terms only occur in the form $\operatorname{rv}_{n}\left(\tau_{i}\right)$ for some $n$. By Lemma 6.3.14 and by compactness, there exists a definable function

$$
\lambda: S \times K^{m+1} \rightarrow S^{\prime} \times S \times K^{m}
$$

with $S^{\prime}$ auxiliary such that the $\operatorname{rv}_{n}\left(\tau_{i}\right)$ are component functions of $\lambda$ (that is, $\lambda$ composed with a coordinate projection gives $\left.\operatorname{rv}_{n}\left(\tau_{i}\right)\right)$ and such that the image of $\lambda$ is given by a valued field quantifier free formula. But then it is easy to construct $\theta$, by quantifying over $S^{\prime}$. This proves the quantifier elimination statement.

Next we prove (b1) and the property about centers. Let $X \subset K$ be an $\mathcal{L}(A)$-definable set, given by a valued field quantifier free $\mathcal{L}(A)$-formula $\varphi(x)$. Let the $f_{i}$ be all the valued field terms appearing in $\varphi$. Lemma 6.3.14 applied to the $f_{i}$ implies (b1) and the property about centers.

Property (b2) follows from the quantifier elimination statement. Namely, consider a valued field quantifier free formula $\varphi$ in one valued field variable $x$ and auxiliary variables $\xi$ running over $S$, giving the graph of a surjection from an auxiliary set $S$ to a ball. Let $\tau_{i}(x)$ be the nonzero valued field terms appearing in $\varphi$. We may suppose that they all appear in the form $\operatorname{rv}_{n}\left(\tau_{i}(x)\right)$ for finitely many $i$, since $\tau_{i}(x)=0$ is equivalent to $\operatorname{rv}_{n}\left(\tau_{i}(x)\right)=0$. Since $\varphi$ associates to any $\xi$ a unique $x$, it follows that $x$ satisfies $\prod_{i} \tau_{i}(x)=0$. Since we may suppose that the $\tau_{i}$ are not identically zero, (b2) follows.

For (b3) one uses Lemma 2.4.4 in Section 2 of [CL2]; property $(*)$ there is clear by looking at quantifier free formulas in two valued field variables which give a definable function, as for (b2). Such a formula $\varphi$ has nonzero valued field terms $\tau_{i}(x, y)$ in the valued field variables $x$ and $y$. We may again suppose that they only appear in the form $\operatorname{rv}_{n}\left(\tau_{i}(x, y)\right)$ for finitely many $n$. Since $\varphi$ describes the graph of a function $x \mapsto y$, this graph must lie in $\prod_{i} \tau_{i}=0$. Now it is clear that either the image is finite, or some fiber is finite. This proves $(*)$ of [CL2] and thus (b3).

The $b$-minimality is proven.

Next we will show that preservation of all balls (in correspondence with the Jacobian) is a consequence of Theorem 6.3.8. Lemmas 6.3.9 and 6.3.15. and a compactness argument. 
Let $F: X \subset K \rightarrow K$ be $A$-definable for some $A$. By Theorem 6.3.8, there exists an $\mathcal{L}_{\text {Hen, } \mathcal{A}}(A)$-definable function $g: X \rightarrow S$ with $S$ auxiliary such that

$$
f(x)=t(x, g(x))
$$

for each $x \in X$ and where $t$ is an $\mathcal{L}_{\text {Hen, } \mathcal{A}}^{*}(A)$-term. By $b$-minimality with centers, we may suppose that $g$ is a $b$-map which has a $B_{n_{0}}$-center $c$ for some $n_{0}$. By Lemma 6.3.15 we may suppose that on each ball $B$ of the form $g^{-1}(s)$ the restriction of $x \mapsto f(x)$ to $B$ is in $\mathcal{O}_{K}^{\dagger}(B)$. By $b$-minimal cell decomposition, we may moreover suppose that $\operatorname{rv}_{n}\left(f^{\prime}(x)\right)$ is constant on each such $B$. Fix a ball $B$ of the form $g^{-1}(s)$, say, of size $\alpha_{B}$, namely of the form $\left\{x: \operatorname{ord}(x-b)>\alpha_{B}\right\}$ for some $b$. Then there exists by Lemma 6.3.9 an integer $n_{B}$ such that the restriction of $a f$ to any ball of size $\alpha_{B}+\operatorname{ord}\left(n_{B}\right)$, contained in $B$, is an isometry, with $a$ any element of the same size as $1 / f^{\prime}$ on $B$. By compactness, there exists a single integer $n$ which can serve for all numbers $n_{B}$ for all such $B$. One can easily refine $g$ so that $c$ becomes a $B_{n \cdot n_{0}}$-center, and each ball $g^{-1}(s)$ is replaced by a union of balls of size ord $(n)$ smaller than $g^{-1}(s)$. The Jacobian property then follows.

Remark 6.3.16. Remark 6.3.10 together with the above proof can be used to get a stronger Jacobian property than the one given in Definition 6.3.6 Namely, given $n>0$, the function $f$ in Definition 6.3.6 can be taken such that for each $s \in f(X)$ such that $f^{-1}(s)$ is a ball, the restriction of $F$ to $f^{-1}(s)$ has the Jacobian property and moreover, for all $s \in f(X)$ and all $x, y \in f^{-1}(s)$,

$$
a(s) \operatorname{rv}_{n}(x-y)=\operatorname{rv}_{n}(F(x)-F(y))
$$

where $\operatorname{rv}_{n} \mathrm{Jac} F$ is constant on $f^{-1}(s)$ and $a(s):=\operatorname{rv}_{n} \operatorname{Jac} F\left(f^{-1}(s)\right)$. This property is a local form of the monotonicity property of [Schi, Section 86].

Proof of Theorem 6.3.8 This proof is similar to that of Theorem 7.2.9 of [CL2] and follows from $b$-minimality with centers and Lemma 6.3.14. Note that it is enough to work piecewise. Namely, with the functions $h_{1,1}$ one can make terms which are characteristic functions of $\operatorname{rv}(1), \operatorname{rv}(2)$, and so on, hence one can always paste together a finite number of terms on finitely many disjoint pieces. For example, to obtain the characteristic function of $Y \subset X$ as a term, define $g(x) \in R V$ as $\operatorname{rv}(1)$ when $x \in Y$ and as $\operatorname{rv}(0)$ else, and let $t(x, \xi)$ be the term $h_{1,1}(1,-1, \xi)$. Note that also the valued field inverse can be given by a term, using $h_{1,1}(1,-y, \operatorname{rv}(1 / y))$. See [CLR1] for more detail.

By Lemma 6.3.14 and compactness as in the proof of Theorem 7.2.9 of [CL2], and with $b$-minimal terminology of [CL2, Sections 3 and 6] about cell decompositions, it follows that a cell decomposition theorem holds where all the centers are given by $\mathcal{L}_{\text {Hen, } \mathcal{A}}^{*}(A)$-terms. Partitioning the graph of $f$ into such cells yields the desired piecewise terms.

Remark 6.3.17. Cell decomposition (as well as other properties) for $\mathcal{T}_{\text {Hen, } \mathcal{A}}$ now follows immediately from [CL2] and the $b$-minimality of $\mathcal{T}_{\text {Hen, } \mathcal{A}}$ established in Theorem 6.3.7. 
Acknowledgments. R. Cluckers was supported as a postdoctoral fellow by the Fund for Scientific Research, Flanders (Belgium) (F.W.O.) and partially by The European Commission, Marie Curie European Individual Fellowship with contract number HPMF CT 2005-007121 during the preparation of this paper.

L. Lipshitz was supported in part by NSF grant DMS-0401175.

The authors acknowledge stimulating conversations with Zach Robinson on some of the subject matter of this paper and thank the University of Leuven and Purdue University for support and hospitality. The research has partially benefitted from the project ANR-06-BLAN-0183.

\section{References}

[Ar] Artin, M.: Algebraic approximation of structures over complete local rings. Publ. Math. I.H.E.S. 36, 23-58 (1969) Zbl 0181.48802 MR 0268188

[B] Bartenwerfer, W.: Die Beschränktheit der Stückzahl der Fasern $K$-analytischer Abbildungen. J. Reine Angew. Math. 416, 49-70 (1991) Zbl 0722.32018 MR 1099945

[BK] Basarab, S., Kuhlmann, F.-V.: An isomorphism theorem for Henselian algebraic extensions of valued fields. Manuscripta Math. 77, 113-126 (1992) Zbl 0773.12004 MR 1283331

[Bo] Bosch, S.: A rigid analytic version of M. Artin's Theorem on analytic equations. Math. Ann. 255, 395-404 (1981) Zbl 0462.14002 MR 0615859

[BGR] Bosch, S., Güntzer, U., Remmert, R.: Non-Archimedean Analysis. Springer (1984) Zbl 0539.14017 MR 0746961

[Ce1] Çelikler, Y. F.: Algebraic properties of separated power series. Math. Z. 259, 681-695 (2008) Zbl 1141.13019 MR 2395133

[Ce2] Çelikler, Y. F.: Quantifier elimination for the theory of algebraically closed valued fields with analytic structure. Math. Logic Quart. 53, 237-246 (2007) Zbl 1120.03021 MR 2330593

[Ce3] Çelikler, Y. F.: On some definable sets over fields with analytic structure. Ann. Pure Appl. Logic 161, 599-616 (2010) MR 2584735

[C] Cluckers, R.: Analytic $p$-adic cell decomposition and integrals. Trans. Amer. Math. Soc. 356, 1489-1499 (2004) Zbl 1048.11094 MR 2034315

[CLR1] Cluckers, R., Lipshitz, L., Robinson, Z.: Analytic cell decomposition and analytic motivic integration. Ann. Sci. École Norm. Sup. (4) 39, 535-568 (2006) Zbl 1168.12006 MR 2290137

[CLR2] Cluckers, R., Lipshitz, L., Robinson, Z.: Real closed fields with nonstandard and standard analytic structure. J. London Math. Soc. (2) 78, 198-212 (2008) Zbl 1184.03032 MR 2427060

[CL1] Cluckers, R., Loeser, F.: Constructible motivic functions and motivic integration. Invent. Math. 173, 23-121 (2008) Zbl 1179.14011 MR 2403394

[CL2] Cluckers, R., Loeser, F.: $b$-minimality. J. Math. Logic 7, 195-227 (2007) Zbl 1146.03021 MR 2423950

[Co] Cohen, P. J.: Decision procedures for real and $p$-adic fields. Comm. Pure Appl. Math. 22, 131-151 (1969) Zbl 0167.01502 MR 0244025

[D1] Denef, J.: The rationality of the Poincare series associated to the $p$-adic points on a variety. Invent. Math. 77, 1-23 (1984) Zbl 0537.12011 MR 0751129

[D2] Denef, J.: $p$-adic semi-algebraic sets and cell decomposition. J. Reine Angew. Math. 369, 154-166 (1986) Zbl 0584.12015 MR 0850632 
[D3] Denef, J.: On the degree of Igusa's local zeta function. Amer. J. Math. 109, 991-1008 (1987) Zbl 0659.14017 MR 0919001

[DD] Denef, J., van den Dries, L.: p-adic and real subanalytic sets. Ann. of Math. 128, 79-138 (1988) Zbl 0693.14012 MR 0951508

[DL1] Denef, J., Lipshitz, L.: Ultraproducts and approximation in local rings. II. Math. Ann. 253, 1-28 (1980) Zbl 0426.13010 MR 0594530

[DL2] Denef, J., Lipshitz, L.: Algebraic power series and diagonals. J. Number Theory 26, 46-67 (1987) Zbl 0609.12020 MR 0883533

[vdD] van den Dries, L.: Analytic Ax-Kochen-Ershov theorems. In: Contemp. Math. 131, Amer. Math. Soc., 379-398 (1992) Zbl 0835.03004 MR 1175894

[DHM] van den Dries, L., Haskell, D., Macpherson, D.: One-dimensional $p$-adic subanalytic sets. J. London Math. Soc. (2) 59, 1-20 (1999) Zbl 0932.03038 MR 1688485

[DMM1] van den Dries, L., Macintyre, A., Marker, D.: The elementary theory of restricted analytic fields with exponentiation. Ann. of Math. 140, 183-205 (1994) Zbl 0837.12006 MR 1289495

[DMM2] van den Dries, L., Macintyre, A., Marker, D.: Logarithmic-exponential power series. J. London Math. Soc. (2) 56, 417-434 (1997) Zbl 0924.12007 MR 1610431

[DMM3] van den Dries, L., Macintyre, A., Marker, D.: Logarithmic-exponential series. Ann. Pure Appl. Logic 111, 61-113 (2001) Zbl 0998.12014 MR 1848569

[FP] Fresnel, J., van der Put, M.: Rigid Geometry and Applications. Birkhäuser (2004) Zbl 1096.14014 MR 2014891

[GR] Gunning, R., Rossi, H.: Analytic Functions of Several Complex Variables. Prentice-Hall (1965) Zbl 0141.08601 MR 0180696

[HP] Hrushovski, E., Peterzil, Y.: A question of van den Dries and a theorem of Lipshitz and Robinson; not everything is standard. J. Symbolic Logic 72, 119-122 (2007) Zbl 1118.03027 MR 2298474

[Kap] Kaplansky, I.: Maximal fields with valuations. I, II. Duke Math. J. 9, 303-321 (1942) and 12, 243-248 (1945) Zbl 0063.03135 (I) Zbl 0061.05506 (II) MR 0006161 (I) MR 0012276(II)

[LL1] Lipshitz, L.: Isolated points on fibers of affinoid varieties. J. Reine Angew. Math. 384, 208-220 (1988) Zbl 0636.14006 MR 0929984

[LL2] Lipshitz, L.: Rigid subanalytic sets. Amer. J. Math. 115, 77-108 (1993) Zbl 0792.14010 MR 1209235

[LR1] Lipshitz, L., Robinson, Z.: Rings of separated power series and quasi-affinoid geometry. Astérisque 264 (2000) Zbl 0957.32011 MR 1758887

[LR2] Lipshitz, L., Robinson, Z.: Model completeness and subanalytic sets. Astérisque 264, 109-126 (2000) Zbl 0957.32011 MR 1758887

[LR3] Lipshitz, L., Robinson, Z.: Uniform properties of rigid subanalytic sets. Trans. Amer. Math. Soc. 357, 4349-4377 (2005) Zbl 1081.03024 MR 2156714

[LR4] Lipshitz, L., Robinson, Z.: Overconvergent real closed quantifier elimination. Bull. London Math. Soc. 38, 897-906 (2006) Zbl 1116.03032 MR 2285243

[Pas1] Pas, J.: Uniform $p$-adic cell decomposition and local zeta-functions. J. Reine Angew. Math. 399, 137-172 (1989) Zbl 0666.12014 MR 1004136

[Pas2] Pas, J.: Cell decomposition and local zeta functions in a tower of unramified extensions of a $p$-adic field. Proc. London Math. Soc. (3) 60, 37-67 (1990) Zbl 0659.12017 MR 1023804

[Pass] Passman, D. S.: The Algebraic Structure of Group Rings. Wiley (1977) Zbl 0368.16003 MR 0470211 
[Po] Poonen, B.: Maximally complete fields. Enseign. Math. 39, 87-106 (1993) Zbl 0807.12006 MR 1225257

[PS] Peterzil, Y., Starchenko, S.: Expansions of algebraically closed fields in o-minimal structures. Selecta Math. 7, 409-445 (2001) Zbl 1010.03027 MR 1868299

[Scan1] Scanlon, T.: Quantifier elimination for the relative Frobenius. In: Valuation Theory and Its Applications, Vol. II, F.-V. Kuhlmann et al. (eds.), Fields Inst. Comm. 33, Amer. Math. Soc., 323-352 (2003) Zbl 1040.03031 MR 2018563

[Scan2] Scanlon, T.: Analytic difference rings. In: Proc. Int. Congress of Mathematicians, Madrid, Vol. II, 71-92 (2006) Zbl 1104.03029 MR 2275589

[Schi] Schikhof, W. H.: Ultrametric Calculus. An Introduction to $p$-adic Analysis. Cambridge Stud. Adv. Math. 4, Cambridge Univ. Press (1984) Zbl 0553.26006 MR 0791759

[S] Schoutens, H.: Rigid subanalytic sets. Compos. Math. 94, 269-295 (1994) Zbl 0867.32012 MR 1310860

[ZS] Zariski, O., Samuel, P.: Commutative Algebra II. Springer (1960) Zbl 0121.27801 MR 0120249 